NASA CONTRACTOR REPORT
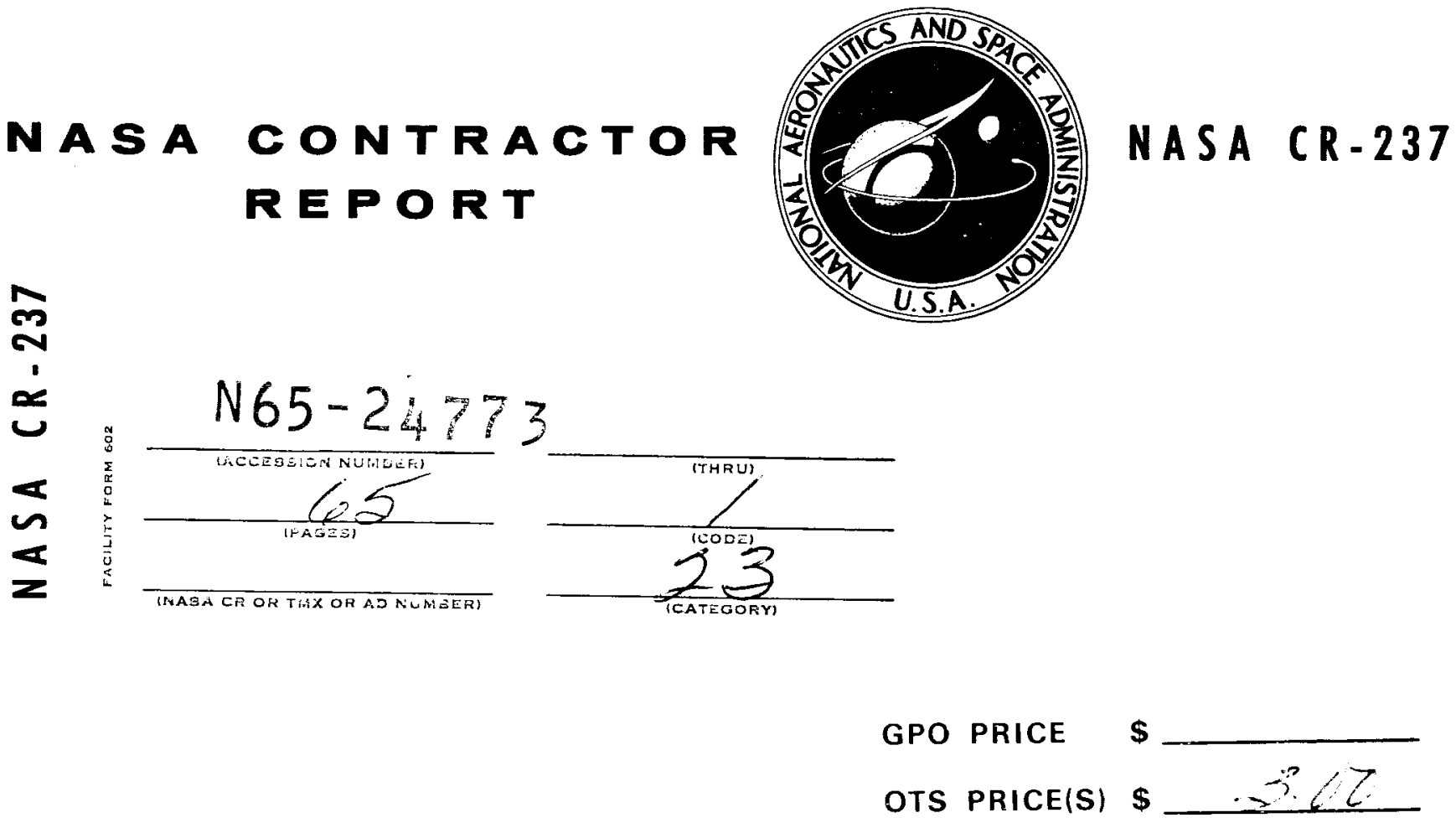

Hard copy $(\mathrm{HC})$

Microfiche (MF)

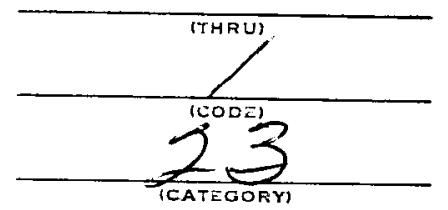

\title{
ABSORPTION OF SOUND IN AIR BELOW 1000 CPS
}

by Cyril M. Harris and W. Tempest

Prepared under Contract No. NAS 8-11002 by

COLUMBIA UNIVERSITY

New York, N. Y.

for

NATIONAL AERONAUTICS AND SPACE ADMINISTRATION • WASHINGTON, D. C. • JUNE 1965 


\section{ABSORPTION OF SOUND IN AIR BELOW 1000 CPS}

By Cyril M. Harris and W. Tempest

Distribution of this report is provided in the interest of information exchange. Responsibility for the contents resides in the author or organization that prepared it.

Prepared under Contract No. NAS 8-11002 by

COLUMBIA UNIVERSITY

New York, N. Y.

for

NATIONAL AERONAUTICS AND SPACE ADMINISTRATION

For sale by the Clearinghouse for Federal Scientific and Technical Information

Springfield, Virginio 22151 - Price $\$ 3.00$ 
TABLE OF CONTENTS

Page

SUMMARY $\ldots \ldots \ldots \ldots \ldots \ldots \ldots \ldots \ldots \ldots$

SECTION I. INTRODUCTION $\ldots \ldots \ldots \ldots \ldots \ldots \ldots \ldots$

SECTION II. MEASUREMENT PROCEDURE. . . . . . . . . . 2

SECTION III. RESULTS OF MEASUREMENTS OF SOUND ABSORPTION

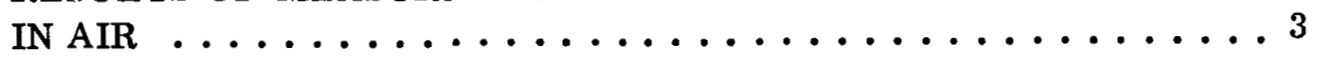

SECTION IV. COMPARISON WITH THEORY $\ldots \ldots \ldots \ldots \ldots \ldots$

SECTION V. ABSORPTION OF SOUND IN OXYGEN-WATER AND OXYGEN-

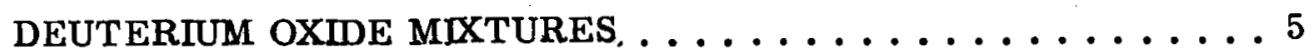

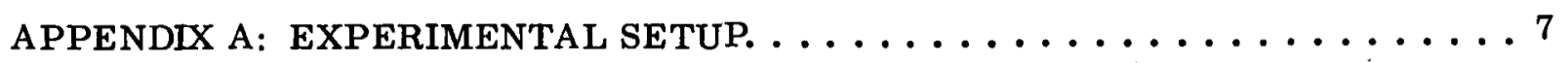

APPENDIX B: TABULAR DATA $\ldots \ldots \ldots \ldots \ldots \ldots \ldots \ldots$

ILLUSTRATIONS . . . . . . . . . . . . . . . . . . 44

REFERENCES. ........................ 58 


\section{LIST OF ILLUSTRATIONS}

1. Attenuation Coefficient $\underline{m}$ versus Percent Relative Humidity for Air at $20^{\circ} \mathrm{C}$ and Normal Atmospheric Pressure . . . . . . . . . . 44

2. Attenuation Coefficient $\underline{m}$ versus Percent Relative Humidity for Air at $0^{\circ} \mathrm{C}$ and Normal $\overline{\mathrm{At} m o s p h e r i c ~ P r e s s u r e ~ . . . . . . . . . . . ~} 45$

3. Attenuation Coefficient $m$ versus Percent Relative Humidity for Air at $-20^{\circ} \mathrm{C}$ and Normal Atmospheric Pressure ........... 46

4. Attenuation Coefficient $\underline{m}$ versus Percent Relative Humidity for Air at $-40^{\circ} \mathrm{C}$ and Normal Atmospheric Pressure ........... 47

5. Plot of Experimental Data at $20^{\circ} \mathrm{C}, 0^{\circ} \mathrm{C}$ and $-20^{\circ} \mathrm{C}$ of Attenuation in Air versus Humidity. These Data Are Presented in Normalized Form $\mathrm{m} / \mathrm{m}_{\max }$ versus $\mathrm{h} / \mathrm{h} \max \ldots \ldots \ldots \ldots \ldots \ldots$

6. Maximum Attenuation Coefficient $m_{\max }$ versus Frequency $\ldots \ldots \ldots$

7, Relaxation Frequency Plotted as a Function of $\underline{h}$, the Percent Molar Concentration of Water Vapor in Air ........... 50

8. Attenuation Coefficient $\mathrm{m}$ versus Percent Molar Concentration of Water Vapor for Oxygen at $20^{\circ} \mathrm{C}$ and Normal Pressure . . . . . 51

9. Relaxation Frequency, $f$ max, Plotted as a Function of $\underline{h}$, the Percent Molar Concentration of Water Vapor in Oxygen . . . . . . 52

10. Attenuation Coefficient $\underline{m}$ versus Percent Relative Humidity . . . . 53 for Air at $20^{\circ} \mathrm{C}$ and Pressure of $400 \mathrm{~mm}$

11. Attenuation Coefficient $\underline{\mathrm{m}}$ versus Percent Relative Humidity for Air at $20^{\circ} \mathrm{C}$ and Pressure of $200 \mathrm{~mm} \ldots \ldots \ldots \ldots \ldots$

12. Attenuation Coefficient $m$ versus Percent Molar Concentration of Deuterium Oxide Vapor for Oxygen at $20^{\circ} \mathrm{C}$ and Normal Pressure $\quad 55$

13. Drawing Showing the Spherical Chamber Used in Measurements. Copper Tubing Attached to Exterior Surface is Part of the Temperature-Control System 


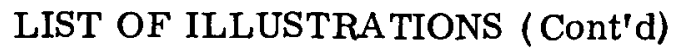

Figure

Title

Page

14. Simplified Schematic Diagram of the Air-Circulation System. Air is Recirculated Continuously Through the Spherical Chamber. The Saturator Either Takes Away Moisture From the Air gr Adds Moisture to It--Depending' on The Relative Temperatures. of the Spherical

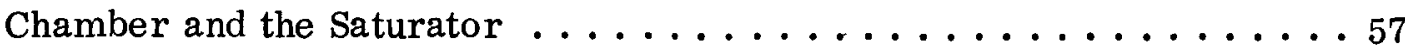




\section{LIST OF TABLES}

Table

Title

Page

1. Tabulation of Original Data Points for Absorption of Sound in Air Versus Relative Humidity at 20 Degrees Centigrade. . . . . . . . . 12

2. Tabulation of Original Data Points for Absorption of Sound in Air Versus Relative Humidity at 0 Degrees Centigrade . . . . . . . . 15

3. Tabulation of Original Data Points for Absorption of Sound in Air Versus Relative Humidity at -20 Degrees Centigrade. . . . . . . 18

4. Tabulation of Original Data Points for Absorption of Sound in Air Versus Relative Humidity at -40 Degrees Centigrade. . . . . . . . 20

5. Tabulation of Data Points for Absorption of Sound in Dry Nitrogen at 20 Degrees Centigrade for the Computation of Wall Losses ..... 21

6A. Tabulation of Original Data Points for Absorption of Sound in Air Versus Relative Humidity at 20 Degrees Centigrade . . . . . . . . 22

6B. Tabulation of Original Data Points for Absorption of Sound in Air Versus Relative Humidity at 20 Degrees Centigrade . . . . . . . . 25

7. Tabulation of Original Data Points for Absorption of Sound in Dry Nitrogen at 20 Degrees Centigrade . . . . . . . . . . . 28

8. Tabulation of Smoothed Data Points for Absorption of Sound in Air Versus Relative Humidity at 20 Degrees Centigrade. . . . . . . . 29

9. Tabulation of Smoothed Data Points for Absorption of Sound in Air Versus Relative Humidity at 0 Degrees Centigrade . . . . . . . . 32

10. Tabulation of Smoothed Data Points for Absorption of Sound in Air Versus Relative Humidity at -20 Degrees Centigrade . . . . . . . 35

11. Tabulation of Smoothed Data Points for Absorption of Sound in Air Versus Relative Humidity at -40 Degrees Centigrade . . . . . . . 37

12. Tabulation of Original Data Points for Absorption of Sound in Oxvgen Versus Relative Humidity at 20 Degrees Centigrade . . . . . . 38 


\section{LIST OF TABLES (Cont'd)}

Table

Title

13. Tabulation of Original Data Points for Absorption of Sound in Oxygen

Versus Concentration of Deuterium Oxide . . . . . . . . . . . . 41 


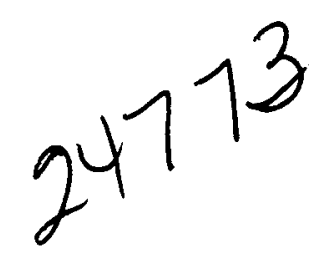

ABSORPTION OF SOUND IN AIR BELOW 1000 CPS

\author{
SUMMARY
}

For many acoustic problems associated with the propagation of sound which is generated by launch vehicles, it is important to have accurate data of absorption of sound in air as a function of atmospheric conditions such as temperature, pressure, and humidity. Accurate data of this type have not been available below $2000 \mathrm{cps}$. Hence the purpose was to extend to lower frequencies the present range of reliable air absorption data. This report presents such information in the frequency range from about $125 \mathrm{cps}$ to $1000 \mathrm{cps}$ at $20^{\circ} \mathrm{C}, 0^{\circ} \mathrm{C},-20^{\circ} \mathrm{C}$, and $-40^{\circ} \mathrm{C}$.

The data described in this report can be applied to studies of acoustic propagation in the atmosphere in addition to the problem of establishing a theoretical model of sound absorption in air. In this connection measurements were made of the absorption in mixtures of oxygen and water vapor and also in mixtures of oxygen and deuterium oxide.

\title{
SECTION I. INTRODUCTION
}

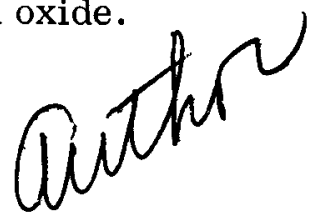

At present it is not possible to make accurate estimates of the attenuation of sound that has propagated through the atmosphere for a considerable distance. Such estimates are of considerable value in many acoustic problems associated with launch vehicles. The total attenuation between two points depends on a number of factors including the absorption of sound in air, refraction, and scattering. It is obvious that reliable data must be available which provide information regarding the absorption of sound in air as a function of atmospheric conditions. Unfortunately, accurate data of this type have not veen available for frequencies below $2000 \mathrm{cps}$. In general, where such data have been required, they have been estimated by extrapolation from measurements at higher frequencies. The purpose of this study was to extend the range over which reliable air absorption data are available to lower frequencies.

During the past six years in this laboratory, an active program of research has been carried out for investigating the absorption of sound (from $125 \mathrm{cps}$ to $12,500 \mathrm{cps}$ ) in air for different conditions of pressure, temperature, and humidity. The results of the measurements in the range from 2000 to $12,500 \mathrm{cps}$ were published in a paper last year [1] . The present report on sound absorption in air provides data in the range from approximately $125 \mathrm{cps}$ to $1000 \mathrm{cps}$. 
In addition to the application of the data described in this report to studies of acoustic propagation in the atmosphere, these data can be applied to the problem of establishing a theoretical model of sound absorption in air. In this connection measurements were made of the absorption of sound in mixtures of oxygen and water vapor and also in mixtures of oxygen and deuterium oxide.

\section{SECTION II. MEASUREMENT PROCEDURE}

A detailed description of the experimental setup used for obtaining the data presented in this report has been given by Harris [1] in a paper which describes the technique of sound absorption measurement employed here. A complete description of the spherical chamber, the electro-acoustic measurement system, the humidity control, and evaluation of wall losses of the spherical chamber, given in that paper, is reproduced in Appendix A. A summary of this information is abstracted in this section.

The spherical chamber in which the measurements are made has an inner diameter of 1.68 meters. It is fabricated of heavy steel in order to minimize the effects of wall losses, which are quite low. For example, the reverberation time at $1000 \mathrm{cps}$ when the chamber is filled with dry air is 43 seconds. Excellent temperature stability of the sphere is obtained and the temperature can be adjusted by pumping a coolant through coils affixed to the exterior surface of the chamber. The spherical chamber and associated equipment can be evacuated before filling the chamber with air to avoid contamination which can have considerable effect on the measurements.

Sound is introduced from a loudspeaker driving unit through a probe tube into the spherical chamber, thereby producing an acoustic point source within the chamber. This source was very poorly matched to the spherical enclosure so that its contribution to the absorption of the chamber wo uld be negligible. The length of the probe was selected so that the point source is near a pressure maximum for the normal modes of vibration of the enclosure that are used in the low frequency measurements (described below), but of such a length as to discriminate against the excitation of nearby normal modes. After an acoustic steady-state has been established by the sound source, the source is turned off and the rate of decay of sound, in decibels per second, is measured.

The method of establishing a steady-state condition of humidity in the spherical enclosure is to pump the air from the sphere through an auxiliary "saturator" chamber which either adds or substracts moisture--depending on the temperature of the saturator. Air is re-circulated through the saturator until the air contains approximately the amount of water vapor desired. Then the saturator is bypassed, but re-circulated until a steadystate humidity condition has been achieved--during this time, water vapor may be absorbed or given off by the steel walls of the spherical chamber. The humidity in the spherical enclosure is measured by an electric hygrometer which uses lithium chloride sensing elements manufactured by Hygrodynamics, Inc. 
Nitrogen has a sound absorption value that is well established. Hence, if the sphere is filled with this gas it is possible to compute the contribution to the measured decay rate $R_{N_{2}}$ that is due to nitrogen along. If decay rate measurements are made in the sphere when filled with nitrogen, the measured value of the decay rate will be greater than $R_{N_{2}}$; the remaining contribution is due to losses at the wall. Now because air and nitrogen are similar in characteristic impedance and in molecular weight, the wall losses are approximately the same for the decay of sound when the sphere is filled with either gas. Thus, decay rate measurements in nitrogen (Tables 5 and 6) provide data for evaluating the wall losses when measurements are made in air. It follows that the decay rate of sound in the sphere, $R_{\text {air }}$, is given by eq. 3 of Appendix A:

$$
R_{\text {air }}=R_{\text {measured (air) }}-R_{\text {measured }\left(N_{2}\right)}+R_{N_{2}}
$$

In this manner the value of the rate of decay for sound in air was evaluated. The attenuation coefficient $\mathrm{m}$ per meter ( as expressed in the equation $\mathrm{I}=\mathrm{I}_{\mathrm{o}} \mathrm{e}^{-\mathrm{mx}}$ ) is related to the decay rate by the equation:

$$
\mathrm{m}=\mathrm{R}_{\text {air }} /(4.34 \mathrm{c}) \text { meters }^{-1}
$$

where $\mathrm{c}$ is the velocity in meters/seconds.

\section{SECTION III. RESULTS OF MEASUREMENTS OF SOUND ABSORPTION IN AIR}

In this report the results of measurement of the absorption of sound in air are given as a function of water vapor content at atmospheric pressure for four temperatures, $20^{\circ} \mathrm{C}, 0^{\circ} \mathrm{C},-20^{\circ} \mathrm{C}$, and $-40^{\circ} \mathrm{C}$. Additional data for air are given at $20^{\circ} \mathrm{C}$ for pressures of $400 \mathrm{~mm}$ and $200 \mathrm{~mm}$. This information is presented in both tabular and graphic form.

The data presented in Reference 1 at higher frequencies were taken by measuring the rate of decay of a large number of modes of vibrations, within a third-octave band, which were excited by a random noise source. Here, in the lower frequency range, the decay rate of individual modes which are excited by a pure tone is measured. Thus, data are given for each of the conditions at the five normal frequencies (eigenfrequencies) of the following normal modes of vibration of the spherical chamber: $(1,1),(0,1),(0,2)$, $(0,3)$, and $(0,4)$. For example, at normal pressure and a temperature of $20^{\circ} \mathrm{C}$, the $(0,1)$ normal mode of vibration, which is the first radial mode, has a normal frequency of $293 \mathrm{cps}$. Since the normal frequencies depend upon the velocity of sound, and since the velocity of sound is a function of temperature, the set of curves presented in Figures 1 through 4 are not at the same set of frequencies for the various temperatures.' 
The actual data points for the absorption measurements taken at atmospheric pressure are given in Tables 1 through 4 along with other system measurements. For application to practical problems, smoothed curves drawn through the actual data points are more useful. Such smoothed curves are shown in Figures 1 through 4 . These data are in good agreement with earlier measurements at higher frequencies [1]. Good accuracy was obtained in all measurements except at $-40^{\circ} \mathrm{C}$ where the attenuation is exceedingly low. For this reason the data at this temperature may be regarded as a best estimate. The data points corresponding to the smoothed curves are given in Tables 8 through 11. Data for reduced air pressure at $20^{\circ} \mathrm{C}$ are shown in Figures 10 and 11 and are listed in Table 6.

\section{SECTION IV. COMPARISON WITH THEORY}

It is of interest to compare the data presented here with the theoretical studies by Kneser [2] of the absorption of sound in air containing water vapor. The first comparison is shown in Figure 5. This curve of normalized attenuation versus normalized humidity was obtained as follows: The original data points for the attenuation coefficient due to molecular absorption are listed in Tables 1 through 4 under "AIR MOL." The data for each curve has a maximum attenuation value $m_{\max }$ at a particular value of water vapor concentration, $h_{\max }$. The data points for each "attenuation versus water vapor" curve were normalized by dividing each value of AIR MOL by $m_{\max }$. According to the theory of Kneser, the normalized data for all frequencies should fall along the dashed curve shown. The solid curve which represents the present results is in close agreement with similar data presented by Harris [1] and with similar data obtained by Delsasso [3] .

According to the theory, the maximum value of absorption increases linearly with frequency. This relationship is shown by the solid lines in Figure 6 for $20^{\circ} \mathrm{C}, 0^{\circ} \mathrm{C}$, and $-20^{\circ} \mathrm{C}$. Also shown are the values of $m_{\max }$ taken from the original data points.

In Figure 7 another comparison with theory is shown in a plot of relaxation frequency, $f_{\max }$, versus the molar concentration of water vapor in the air. The relaxation frequency for a given condition of humidity is the frequency of maximum absorption and is related to the angular relaxation frequency by the equation

$$
\mathrm{k}=2 \pi \mathrm{f}_{\max }=\omega_{\max }
$$

The data obtained in this study are plotted together with similar data from Reference 1 obtained at higher frequencies. 


\section{SECTION V, ABSORPTION OF SOUND IN OXYGEN-WATER AND OXYGEN-DEUTERIUM OXIDE MIXTURES}

In the past, a number of experiments have been performed to determine the absorption of sound in dry oxygen and in oxygen containing water vapor [4,11]. It has been shown that there is a peak in the curve of "sound absorption versus moisture content" due to the relaxation of the internal energy of the vibrational mode of the oxygen molecule. In the dry gas, recent work (Parker [7], Holmes, Smith and Tempest [8]) has shown the peak in absorption to occur at about $9 \mathrm{cps}$ at a pressure of $1 \mathrm{~atm}$ and at a temperature of $20^{\circ} \mathrm{C}$. Other work indicated a higher relaxation frequency (Knotzel and Knotzel [6], $50 \mathrm{cps}$; and Henderson [9], $60 \mathrm{cps}$ ). As is the case with air-water mixtures, measurements in oxygen-water mixtures have shown that small quantities of water significantly affect the relaxation frequency and that this frequency of maximum absorption rises rapidly with increasing moisture content.

Measurements are reported here of the absorption of sound in extremely-dry oxygen of high purity, as a function of water vapor content. Figure 8 shows the results plotted in the form of intensity attenuation coefficient $m$ per meter as a function of moisture content in percent molar concentration of water for the following frequencies (the pairs of numbers in the brackets specify the normal mode of vibration): $[(1,1), 130 \mathrm{cps} ;(0,1)$, $280 \mathrm{cps} ;(0,2), 482 \mathrm{cps} ;(0,3), 680 \mathrm{cps} ;(0,4), 878 \mathrm{cps}]$. These data are tabulated in Table 12. The moisture content at which the peak in the absorption curve occurs, increases with increasing frequency. The expected values of maximum absorption at the various frequencies were calculated from the Kneser theory [2], and were found to be on average about 5 percent higher than the measured values.

Figure 9 shows a comparison between the experimental data presented here and data of other researchers. Four curves are plotted of relaxation frequency as a function of moisture content as calculated from the following equations which are given in their respective papers:

$\begin{array}{llr}\text { Knudsen and Obert [5] } & \mathrm{f}_{\max }= & 4.96 \times 10^{2} \mathrm{~h}+6.05 \times 10^{3} \mathrm{~h}^{2} \\ \text { Knotzel and Knotzel [6] } & \mathrm{f}_{\max }= & 40+1.95 \times 10^{3} \mathrm{~h}+1.32 \times 10^{4} \mathrm{~h}^{2} \\ \text { Clark and Henderson [10] } & \mathrm{f}_{\max }= & 3+1.66 \times 10^{3} \mathrm{~h}+1.45 \times 10^{4} \mathrm{~h}^{2} \\ \text { Harlow and Kitching [11] } & \mathrm{f}_{\max }= & 2.10 \times 10^{2} \mathrm{~h} \times 1.20 \times 10^{4} \mathrm{~h}^{2}\end{array}$

where $f_{\max }$ is the frequency of maximum absorption in cps and $h$ is the percent molar concentration of water. The data of Knudsen and Obert, which differs considerably from the other results shown, is extrapolated from measurements at 3,000 cps and higher; it is probably subject to considerable error at low frequencies. In contrast, the data of 
Harlow and Kitching is based on measurements at frequencies as low as $98 \mathrm{cps}$, which rules out error due to extrapolation. It is possible that the difference between their results and those of others may be due to the method by which they determined the moisture content in their gas. Before the air re-circulation system shown in Figure 14 (Appendix A) was developed for humidity control, some measurements were made in which moisture content was determined from weights of evaporated water in the system. Results so obtained were compared with results in which humidity is measured by the direct technique of circulating the gas over calibrated electrical conductivity elements. It was found that the two methods gave quite different results, in terms of the moisture content required to produce a particular frequency of maximum absorption, with the evaporation technique giving humidity levels as high as three times the direct measurements. It was concluded that a considerable amount of moisture may be taken out of the gas in the chamber by the walls. Such an effect would result in an apparently lower measured value of relaxation frequency for a given moisture content as reported by Harlow and Kitching. The results of the present study shown by the $x$ 's in Figure 9 are in good agreement with those of Knotzel and Knotzel; they are in very close agreement with the data of Clark and Henderson, thus supporting the view that the frequency dependence of the absorption peak on moisture content contains a quadratic term.

Data are shown in Figure 12 for the absorption of sound in a mixture of oxygen and deuterium oxide. (These data are tabulated in Table 13.) A comparison of Figures 8 and 12 indicates that for a given frequency, the maximum value of absorption in oxygenwater mixture is approximately the same as the maximum value for water vapor and deuterium oxide mixture. However, the curves for the oxygen-deuterium oxide mixture have their peaks at significantly lower vapor concentrations. At present there is no satisfactory theoretical model to explain these results but these data may prove useful in helping to provide the necessary information required in establishing such a model. 


\section{APPENDIX A}

\section{EXPERIMENTAL SETUP ${ }^{1}$}

Spherical Chamber

The spherical chamber used in this study has an inner diameter of $1.68 \mathrm{~m}$ ( olume $=2.48 \mathrm{~m}^{3}$ ). It was specially fabricated, in two hemispherical shells, from hot<rolled steel having a thickness of $16 \mathrm{~mm}$. The two halves are fitted with flanges and bolted together with a Teflon gasket, as illustrated in Figure 13. That the acoustic boundary losses are low in this chamber is illustrated by the fact that at $1000 \mathrm{cps}$ its reverberation time is $43 \mathrm{sec}$ when the chamber is filled with dry air. Measurements of the decay of sound in the chamber, when it is filled with nitrogen, show that there are no isolated mechanical resonances of the spherical chamber housing which have significant effect on the rate of decay of sound in the enclosure over the frequency range employed. The entire chamber is packed in glass-fiber blankets to provide thermal insulation. Its temperature is controlled by pumping a methanol coolant through copper tubing fastened to the exterior surface of the sphere (Fig. 13). By this means the air temperature within the chamber can be set at any value between $20^{\circ}$ and $-60^{\circ} \mathrm{C}$ and can be held constant to within $\pm 0.1^{\circ} \mathrm{C}$.

A high-capacity vacuum pump connected to the chamber, together with a diffusion pump, can reduce the pressure within the sphere and its associated air lines to 1 micron (mercury column height). This is essential in order to free the entire system from contamination and to rid all parts of the system of water vapor which may be absorbed by the interior walls of the sphere and walls of the air-circulation system. During the actual decay measurements, the air-circulation system is not in operation; then the lines are closed by gate valves to avoid the loss of acoustic energy from the spherical chamber to the lines.

\section{Electro-Acoustic Measurement System}

The sound source is a 60-watt loudspeaker unit that is coupled to the spherical chamber by a stainless steel probe tube, $1 / 4$ inch in diameter, as illustrated in Figure 13. This arrangement provides an effective acoustic point source within the chamber at the end of the probe tube. The electrical and acoustical coupling of the acoustic source are purposely mismatched in impedance so that the amount of acoustic energy that

1

The material contained in this Appendix is taken from the reprint "Absorption of Sound in Air in the Audio-Frequency Range," Cyril M. Harris, Journal of the Acoustical Society of America, Vol. 35, No. 1, pp. 11-17, January 1963, Reference 1. 
is absorbed by this transducer, while it is inactive during decay measurement, will not be significant. The loudspeaker is driven from a random-noise source. A small dynamic microphone is located in the wall of the spherical chamber. The output of the microphene is amplified, fed through a Bruel and Kjaer third-octave filter (type 2109), and thence to a high-speed level recorder. When the random-noise source is turned off, a curve of the rate of decay of sound in the spherical chamber is obtained with the level recorder. The slope of this decay curve determines the decay rate in $\mathrm{db} / \mathrm{sec}$ at the center frequency of the band at which the third-octave analyzer is set.

\section{Humidity Control}

The problem of accurately controlling and measuring the humidity in a chamber in which air absorption measurements are made has always presented difficulties. In past studies, the accuracy of humidity-measurement techniques at low values of humidity, a range which is often of considerable interest, has been poor. In addition to the question of accuracy, there is the problem of ensuring that the humidity measured actually is representative of conditions within the chamber. Difficulties arise because of the absorption of water by surfaces within the measurement system.

Humidity control and measurement probably account for a major source of discrepancy among published data on the absorption of sound in air. The method used here for establishing controlled conditions of humidity is illustrated in Figure 14. Air is circulated through a closed system by means of a circulation pump which consists of a small high-speed turbofan. Air leaves the spherical chamber through an outlet at the bottom of the sphere. Then it passes through a "saturator" which is a small stainless steel cylinder whose temperature can be controlled from approximately $-60^{\circ} \mathrm{C}$ to $+20^{\circ} \mathrm{C}$ by means of a coolant in which the saturator is immersed. Distilled water is contained in the bottom of the saturator. Moisture either is taken from the air that passes through the saturator and deposited in the saturator, or is taken from the saturator and added to the air that passes through the saturator -- depending upon the relative temperatures of the spherical chamber and the saturator. Air is recirculated through the system until the air in the spherical chamber contains approximately the amount of water vapor required to achieve the desired equilibrium condition. Then the saturator is bypassed (by a valve system that is not shown in Fig. 14); this causes the air to re-circulate from the sphere, through the pump, and then back to the sphere -- until a steady-state humidity condition is obtained. This usually requires about a half hour.

Two sets of electric hygrometers were employed to measure relative humidity. The operation of the hygrometers is based upon the change in the resistance, with humidity, of lithium chloride sensing elements (class $A$, type $H$ ) which are in a bridge circuit and are manufactured by Hygrodynamics, Inc. Under the conditions employed, these elements have a rapid response time and provide a continuous monitoring of the humidity within the spherical chamber during conditions of re-circulation of air. The individual sensing elements in the two sets covered the following ranges: 1.6 to $5 \%, 5$ to $14 \%, 12$ 
to $20 \%, 18$ to $30 \%, 29$ to $43 \%, 41$ to $59 \%, 54$ to $72 \%$. The two sets were closely matched against each other. One set was placed at the air inlet near the top of the spherical chamber and the other at the air outlet at the bottom. Observations of the readings of these sets of elements were used to determine when equilibrium was achieved. These humiditysensing elements were calibrated in the laboratory of the manufacturer immediately before the data contained in this paper were taken--then all units were calibrated once again in a similar manner directly after the experimental data were taken. Essentially, a substitution method of humidity calibration was employed so that the accuracy provided by the sensing elements was greater than that usually quoted for such units which are used under varying field conditions -- here the accuracy was better than $\pm 1 \% \mathrm{RH}$ (relative humidity) except in the lowest range where it was about $\pm 0.5 \% \mathrm{RH}$.

Two calibration techniques are employed by the manufacturer of the humiditysensing elements that were used. Above $5 \% \mathrm{RH}$, elements are calibrated in a controlledhumidity chamber using a high-precision psychrometer employing thermometers calibrated by the National Bureau of Standards. These psychrometric readings are referred to relative-humidity tables based on the barometric pressure corresponding to that in the calibration chamber. In the very low humidity range, elements are calibrated using a two-pressure technique embodying the principles outlined by Weaver and Riley in which a known humidity condition is generated by saturating a gas stream at elevated pressures, and then expanding to atmospheric pressure. Calibrations of the sensing elements used in this study are reproducible within $\pm 0.2 \% \mathrm{RH}$. The two techniques are compared at the lower-humidity ranges and are in close agreement (within $\pm 0.5 \% \mathrm{RH}$ ).

\section{Evaluation of Wall Losses of Spherical Chamber}

In order to determine the absorption of sound in air from measurements of the rate of decay of sound in the spherical chamber used in this study, it is necessary to know the extent of the contribution to the rate of decay that may be attributed to wall losses. This may be evaluated from measurements of the rate of decay of sound in the chamber when it is filled with nitrogen since nitrogen exhibits no anomalous absorption in the frequency range of measurement. By comparing the measured values of decay rate in nitrogen with the decay rate computed from absorption data for nitrogen, one obtains a small difference which represents the effects of wall losses. This is shown as follows: The decay rate of sound, $R_{\text {measured }}$ (air), that one measures in the spherical chamber when it is filled with air is given (in decibels per second) by

$$
\mathrm{R}_{\text {measured (air) }}=\mathrm{R}_{\text {air }}+\mathrm{R}_{\text {wall, }}
$$

where $R_{\text {air }}$ is the decay rate due to absorption in the air, and $R_{w a l l}$ is the decay rate due to absorption at the walls. 
When the chamber is filled with prepurified dry nitrogen,

$$
R_{\text {measured }\left(\mathrm{N}_{2}\right)}=\mathrm{R}_{\mathrm{N}_{2}}+\mathrm{R}_{\text {wall, }}
$$

where $\mathrm{R}_{\mathrm{N}_{2}}$ is the decay rate due to absorption in nitrogen in db/sec.

If it is assumed that the wall losses for $\mathrm{N}_{2}$ and air are approximately the same, because these gases are closely similar in molecular weight and characteristic impedance, then subtracting eq. 2 from eq. 1 :

$$
R_{\text {air }}=R_{\text {measured (air) }}-R_{\text {measured }\left(N_{2}\right)}+R_{N_{2}}
$$

The first two terms on the right are obtained from measurements of the rate of decay of sound in the spherical chamber while the third term is calculated from the data for nitrogen by Parbrook and Tempest. Consideration has been given to possible variation in the boundary losses with changes in the humidity within the sphere. As pointed out by Evans and Bazley in discussing this possibility, the work of Knudsen, Wilson and Anderson indicates that such an effect is not significant; their data show that the re is no appreciable change in wall absorption even when moisture condenses on the wall surfaces.

The value of $R_{a i r}$ in $\mathrm{db} / \mathrm{sec}$ given by eq. 3 is converted to the attenuation coefficient $\mathrm{m}$ per meter as expressed in the equation $\mathrm{I}=\mathrm{I}_{0} \mathrm{e}^{-\mathrm{mx}}$ by the relation

$$
\mathrm{m}=\mathrm{R}_{\text {air }} /(4.34 \mathrm{c}) \text { meters }^{-1}
$$

where $c$ is the velocity of sound in $\dot{\mathrm{m}} / \mathrm{sec}$. 
APPENDIX 3

TABULAR DATA *

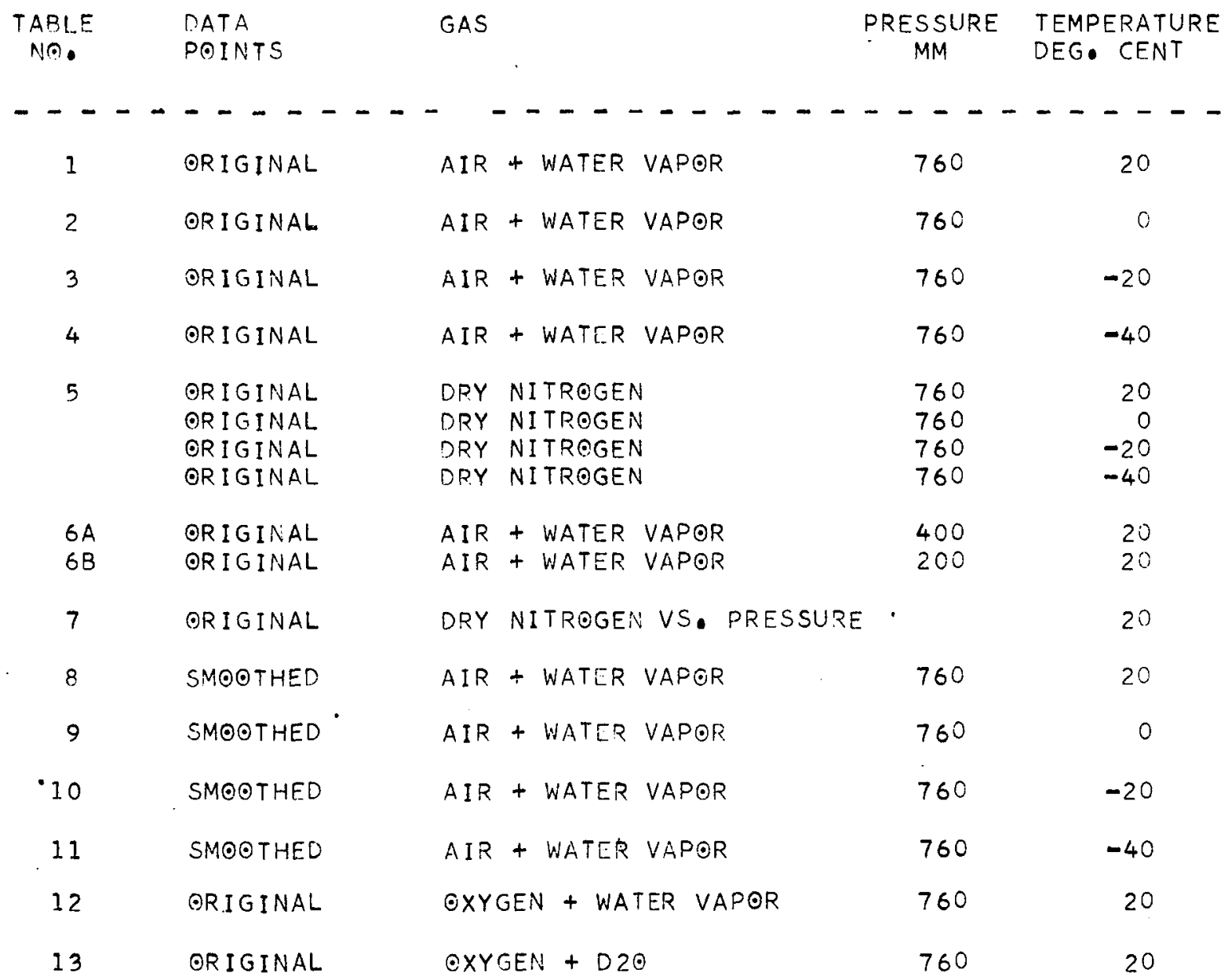

* These tabular data, actually computer run sheets, are graphically illustrated in various figures from 1 through 12 . 
TABLE NO. 1

TABULATION OF ORIGINAL DATA POINTS FOR

ABSORPTION OF SOUND IN AIR VERSUS RELATIVE HUMIDITY AT 20 DEGREES CENTIGRADE

\begin{tabular}{|c|c|c|c|c|c|c|c|c|c|c|c|}
\hline $\mathrm{R} \bullet \mathrm{H} \bullet$ & & FREQ & LAMDA & $\begin{array}{c}\text { AIR } \\
+\end{array}$ & & $\begin{array}{l}\text { AIR } \\
\text { ONLY }\end{array}$ & $\begin{array}{l}\text { AIR } \\
\text { ONLY }\end{array}$ & $\begin{array}{l}\text { AIR } \\
\text { CLAS }\end{array}$ & $\begin{array}{l}\text { AIR } \\
M \odot L\end{array}$ & MU & $\begin{array}{l}3000 * \\
\text { LOG }\end{array}$ \\
\hline PRCT & $\begin{array}{l}\text { DEG } \\
\text { CENT } \\
1\end{array}$ & CPS & $\begin{array}{l}\text { METRS } \\
2 \\
901234\end{array}$ & $\begin{array}{l}\text { WALL } \\
\text { DB/S } \\
3 \\
901236\end{array}$ & $\begin{array}{l}\text { WALL } \\
\mathrm{DB} / \mathrm{S}\end{array}$ & $\begin{array}{l}D B / S \\
4 \\
901234\end{array}$ & $/ M$ & $5^{I M}$ & $/ M$ & 0 & $\mathrm{RH}$ \\
\hline
\end{tabular}

$\begin{array}{rrrr}1.1 & 20 & 136 & 2.520 \\ 1.2 & 20 & 136 & 2.520 \\ 1.4 & 20 & 136 & 2.520 \\ 1.8 & 20 & 136 & 2.520 \\ 2.1 & 20 & 136 & 2.520 \\ 2.7 & 20 & 136 & 2.520 \\ 3.8 & 20 & 136 & 2.520 \\ 5.1 & 20 & 136 & 2.520 \\ 6.1 & 20 & 136 & 2.520 \\ 6.2 & 20 & 136 & 2.520 \\ 7.2 & 20 & 136 & 2.520 \\ 7.4 & 20 & 136 & 2.520 \\ 8.2 & 20 & 136 & 2.520 \\ 9.0 & 20 & 136 & 2.520 \\ 10.1 & 20 & 136 & 2.520 \\ 13.3 & 20 & 136 & 2.520 \\ 16.3 & 20 & 136 & 2.520 \\ 20.0 & 20 & 136 & 2.520 \\ 25.4 & 20 & 136 & 2.520 \\ 31.0 & 20 & 136 & 2.520 \\ 39.0 & 20 & 136 & 2.520\end{array}$

$\begin{array}{rrrrrrrr}2.54 & 1.99 & .55 & 0037 & 0007 & 0037 & 0093 & -2999 \\ 3.18 & 1.99 & 1.19 & 0080 & 0007 & 0080 & 0202 & 238 \\ 3.08 & 1.99 & 1.09 & 0073 & 0007 & 0073 & 0184 & 438 \\ 2.76 & 1.99 & .77 & 0052 & 0007 & 0052 & 0131 & 766 \\ 2.64 & 1.99 & .65 & 0044 & 0007 & 0044 & 0111 & 967 \\ 2.50 & 1.99 & .51 & 0034 & 0007 & 0034 & 0086 & 1294 \\ 2.36 & 1.99 & .37 & 0025 & 0007 & 0025 & 0063 & 1739 \\ 2.30 & 1.99 & .31 & 0021 & 0007 & 0021 & 0053 & 2123 \\ 2.26 & 1.99 & .27 & 0018 & 0007 & 0018 & 0045 & 2356 \\ 2.36 & 1.99 & .37 & 0025 & 0007 & 0025 & 0063 & 2377 \\ 2.32 & 1.99 & .33 & 0022 & 0007 & 0022 & 0056 & 2572 \\ 2.26 & 1.99 & .27 & 0018 & 0007 & 0018 & 0045 & 2608 \\ 2.35 & 1.99 & .36 & 0024 & 0007 & 0024 & 0061 & 2741 \\ 2.22 & 1.99 & .23 & 0015 & 0007 & 0015 & 0038 & 2863 \\ 2.26 & 1.99 & .27 & 0018 & 0007 & 0018 & 0045 & 3013 \\ 2.38 & 1.99 & .39 & 0026 & 0007 & 0026 & 0066 & 3372 \\ 2.35 & 1.99 & .36 & 0024 & 0007 & 0024 & 0061 & 3637 \\ 2.36 & 1.99 & .37 & 0025 & 0007 & 0025 & 0063 & 3903 \\ 2.23 & 1.99 & .24 & 0016 & 0007 & 0016 & 0040 & 4215 \\ 2.22 & 1.99 & .23 & 0015 & 0007 & 0015 & 0038 & 4474 \\ 2.22 & 1.99 & .23 & 0015 & 0007 & 0015 & 0038 & 4773\end{array}$

$\begin{array}{rrrr}.1 & 20 & 293 & 1.170 \\ 1.2 & 20 & 293 & 1.170 \\ 1.4 & 20 & 293 & 1.170 \\ 1.8 & 20 & 293 & 1.170 \\ 2.1 & 20 & 293 & 1.170 \\ 2.7 & 20 & 293 & 1.170 \\ 3.8 & 20 & 293 & 1.170 \\ 5.1 & 20 & 293 & 1.170 \\ 6.1 & 20 & 293 & 1.170 \\ 6.2 & 20 & 293 & 1.170 \\ 7.2 & 20 & 293 & 1.170 \\ 7.4 & 20 & 293 & 1.170 \\ 8.2 & 20 & 293 & 1.170 \\ 9.0 & 20 & 293 & 1.170 \\ 10.1 & 20 & 293 & 1.170 \\ 13.3 & 20 & 293 & 1.170 \\ 16.3 & 20 & 293 & 1.170 \\ 20.0 & 20 & 293 & 1.170 \\ 25.4 & 20 & 293 & 1.170 \\ 31.0 & 20 & 293 & 1.170 \\ 39.0 & 20 & 293 & 1.170\end{array}$

$\begin{array}{rrrrrrrr}1.95 & 1.36 & .59 & 0040 & 0032 & 0040 & 0047-2999 \\ 4.05 & 1.36 & 2.69 & 0181 & 0032 & 0181 & 0212 & 238 \\ 4.17 & 1.36 & 2.81 & 0189 & 0032 & 0189 & 0221 & 438 \\ 4.06 & 1.36 & 2.70 & 0181 & 0032 & 0181 & 0212 & 766 \\ 3.70 & 1.36 & 2.34 & 0157 & 0032 & 0157 & 0184 & 967 \\ 3.18 & 1.36 & 1.82 & 0122 & 0032 & 0122 & 0143 & 1294 \\ 2.86 & 1.36 & 1.50 & 0101 & 0032 & 0101 & 0118 & 1739 \\ 2.43 & 1.36 & 1.07 & 0072 & 0032 & 0072 & 0084 & 2123 \\ 2.22 & 1.36 & .86 & 0058 & 0032 & 0058 & 0068 & 2356 \\ 2.22 & 1.36 & .86 & 0058 & 0032 & 0058 & 0068 & 2377 \\ 2.12 & 1.36 & .76 & 0051 & 0032 & 0051 & 0060 & 2572 \\ 2.09 & 1.36 & .73 & 0049 & 0032 & 0049 & 0057 & 2608 \\ 2.07 & 1.36 & .71 & 0048 & 0032 & 0048 & 0056 & 2741 \\ 2.01 & 1.36 & .65 & 0044 & 0032 & 0044 & 0052 & 2863 \\ 2.03 & 1.36 & .67 & 0045 & 0032 & 0045 & 0053 & 3013 \\ 1.99 & 1.36 & .63 & 0042 & 0032 & 0042 & 0049 & 3372 \\ 1.98 & 1.36 & .62 & 0042 & 0032 & 0042 & 0049 & 3637 \\ 1.98 & 1.36 & .62 & 0042 & 0032 & 0042 & 0049 & 3903 \\ 1.97 & 1.36 & .61 & 0041 & 0032 & 0041 & 0048 & 4215 \\ 1.98 & 1.36 & .62 & 0042 & 0032 & 0042 & 0049 & 4474 \\ 1.96 & 1.36 & .60 & 0040 & 0032 & 0040 & 0047 & 4773\end{array}$


TABLE NO. 1

TABULATION OF ORIGINAL DATA POINTS FOR

ABSORPTION OF SOUND IN AIR VERSUS RELATIVE HUMIDITY AT 20 DEGREES CENTIGRADE

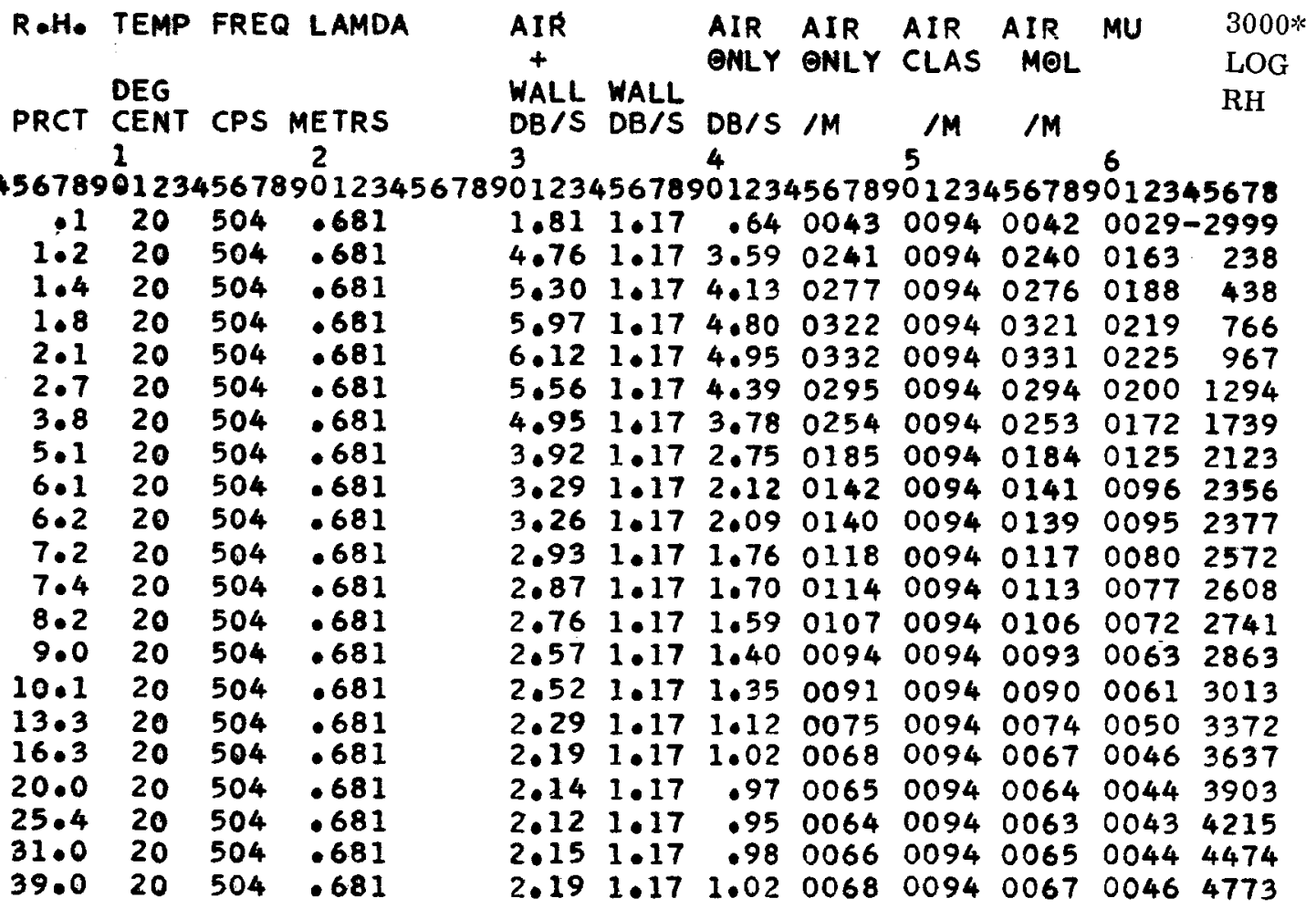

\begin{tabular}{|c|c|c|c|}
\hline $\begin{array}{l}.1 \\
1.2 \\
1.4 \\
1.8 \\
2.1 \\
2.7 \\
3.8 \\
5.1 \\
6.1 \\
6.2 \\
7.2 \\
7.4 \\
8.2 \\
9.0 \\
10.1\end{array}$ & $\begin{array}{l}20 \\
20 \\
20 \\
20 \\
20 \\
20 \\
20 \\
20 \\
20 \\
20 \\
20 \\
20 \\
20 \\
20 \\
20 \\
20 \\
20 \\
20 \\
20 \\
20 \\
20\end{array}$ & $\begin{array}{l}712 \\
712 \\
712 \\
712 \\
712 \\
712 \\
712 \\
712 \\
712 \\
712 \\
712 \\
712 \\
712 \\
712 \\
712 \\
712 \\
712 \\
712 \\
712 \\
712 \\
712\end{array}$ & 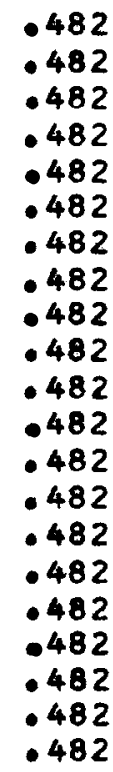 \\
\hline
\end{tabular}

$\begin{array}{lllllllr}2.77 & 2.07 & .70 & 0047 & 0187 & 0045 & 0022-2999 \\ 6.22 & 2.07 & 4.15 & 0279 & 0187 & 0277 & 0134 & 238 \\ 6.97 & 2.07 & 4.90 & 0329 & 0187 & 0327 & 0158 & 438 \\ 8.30 & 2.07 & 6.23 & 0418 & 0187 & 0416 & 0201 & 766 \\ 9.13 & 2.07 & 7.06 & 0474 & 0187 & 0472 & 0228 & 967 \\ 9.26 & 2.07 & 7.19 & 0483 & 0187 & 0481 & 0232 & 1294 \\ 8.73 & 2.07 & 6.66 & 0447 & 0187 & 0445 & 0215 & 1739 \\ 7.30 & 2.07 & 5.23 & 0351 & 0187 & 0349 & 0168 & 2123 \\ 6.21 & 2.07 & 4.14 & 0278 & 0187 & 0276 & 0133 & 2356 \\ 6.19 & 2.07 & 4.12 & 0277 & 0187 & 0275 & 0133 & 2377 \\ 5.54 & 2.07 & 3.47 & 0233 & 0187 & 0231 & 0111 & 2572 \\ 5.41 & 2.07 & 3.34 & 0224 & 0187 & 0222 & 0107 & 2608 \\ 5.13 & 2.07 & 3.06 & 0205 & 0187 & 0203 & 0098 & 2741 \\ 4.79 & 2.07 & 2.72 & 0183 & 0187 & 0181 & 0087 & 2863 \\ 4.74 & 2.07 & 2.67 & 0179 & 0187 & 0177 & 0085 & 3013 \\ 4.15 & 2.07 & 2.08 & 0140 & 0187 & 0138 & 0067 & 3372 \\ 3.89 & 2.07 & 1.82 & 0122 & 0187 & 0120 & 0058 & 3637 \\ 3.72 & 2.07 & 1.65 & 0111 & 0187 & 0109 & 0053 & 3903 \\ 3.62 & 2.07 & 1.55 & 0104 & 0187 & 0102 & 0049 & 4215 \\ 3.61 & 2.07 & 1.54 & 0103 & 0187 & 0101 & 0049 & 4474 \\ 3.65 & 2.07 & 1.58 & 0106 & 0187 & 0104 & 0050 & 4773\end{array}$

* last column employed in computer plotting only. 
TAELE NO. 1

TAOULATIEN SF ORIGINAL DATA PEINTS FET

ABSORPTION OF SOUND IN AIR VERSUS RELATIVE HUAIIITY AT 20 DEGREES CENT IGRADE

R.H. TEMP FREQ LAVBA DEG

PRCT CENT CPS METRÖ 1

$$
2
$$

A I

$+$

WALL WALL

AIR AIR AIR AIR HU

3000*'s DOIS DR/S

ONLY ONLY CLAS YOL

LOG

RH

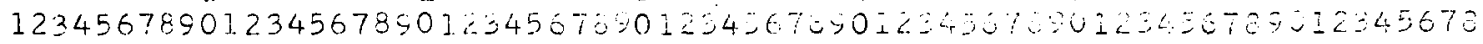

\begin{tabular}{|c|c|c|c|c|c|c|c|c|c|c|c|}
\hline .1 & 20 & 918 & .374 & 5.22 & 4.20 & .72 & $004 i$ & 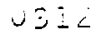 & 0043 & 401 & \\
\hline & 20 & 918 & .374 & 8.55 & 4.30 & 4.05 & 0272 & $\cos 12$ & $0 \% 69$ & $C 101$ & 232 \\
\hline . & 20 & 918 & .374 & 9.26 & 4.50 & 4.76 & 0320 & 0322 & $33: 7$ & $011 \%$ & 433 \\
\hline & 20 & 918 & .374 & II.00 & 4.50 & 6.50 & 0436 & $\cup 312$ & 0433 & $\cup 162$ & 766 \\
\hline & 20 & 918 & .374 & 12.40 & 4.50 & 7.90 & 0530 & $\cup 312$ & $\cup 527$ & 4197 & 96 \\
\hline$\cdot 7$ & 20 & 918 & - 374 & 13.35 & 4.50 & 8.85 & 0594 & 0312 & 0591 & 0221 & 294 \\
\hline 3.5 & 20 & 918 & .274 & 12.20 & 4.50 & 8.70 & 0584 & 0312 & 0581 & 227 & 21 \\
\hline & 20 & 918 & .374 & 11.70 & 4.50 & 7.20 & 0483 & 0312 & 0480 & 0179 & 21.5 \\
\hline . 1 & 20 & 918 & .374 & 10.35 & 4.50 & 5.85 & 0393 & 0312 & 3 & 0146 & \\
\hline $6 \cdot 2$ & 20 & 918 & .374 & 10.30 & 4.50 & 5.80 & 0389 & 312 & 38.6 & 0144 & 237 \\
\hline $7 \cdot 2$ & 20 & 918 & .374 & & 4.50 & 4.48 & 0334 & 312 & 31 & 124 & 25 \\
\hline & 20 & 918 & .374 & 9 & 4.50 & 4.76 & 0320 & 0312 & 17 & 119 & $26 ?$ \\
\hline $8 . ?$ & 20 & 918 & .374 & & 4.50 & 4.35 & 0292 & & & 108 & 272 \\
\hline 9.0 & 20 & 518 & .374 & & 4.50 & 3.83 & 0257 & 12 & 54 & 095 & 28 \\
\hline 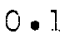 & 20 & 918 & .374 & & & & $025 ?$ & & & 93 & \\
\hline & 20 & 918 & .374 & 7 & 4.50 & 2.75 & 0185 & 12 & 2 & 0069 & 32 \\
\hline 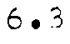 & 20 & 918 & .374 & 6.90 & 4.50 & 2.42 & 0101 & 3212 & 58 & 0056 & 363 \\
\hline & 20 & 918 & .374 & 6.50 & $4 \cdot 30$ & 2.00 & 0134 & $03 i$ & & $60 \ldots$ & $3: 6$ \\
\hline .4 & 20 & 918 & .374 & 6.28 & 4.30 & $=.70$ & $011 \%$ & 0322 & 025 & $\therefore 42$ & $C_{T} Z 7$ \\
\hline & 20 & 918 & .374 & & 4.50 & 1.73 & $0 \pm 15$ & & & $\because \ldots$ & 24 \\
\hline & 20 & 91 & .374 & 6 & 4.50 & $i .78$ & 0129 & 0312 & 1.6 & 0043 & 477 \\
\hline 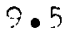 & 20 & 918 & .374 & 6. & 4.50 & 1.53 & 0303 & & & 0.27 & 47 \\
\hline
\end{tabular}

* last column employed in computer plotting only. 
TABLE NO. 2

TABULATION OF ORIGINAL DATA POINTS FOR

ABSORPTION OF SOUND IN AIR VERSUS RELATIVE HUMIDITY AT O DEGREES CENT IGRADE

\begin{tabular}{|c|c|c|c|c|c|c|c|c|c|c|c|}
\hline R•H• & TEMP & FREQ & 2 LAMOA & $\begin{array}{c}\text { AIR } \\
+\end{array}$ & & $\begin{array}{l}\text { AIR } \\
\text { ONLY }\end{array}$ & $\begin{array}{l}\text { AIR } \\
\text { ONLY }\end{array}$ & $\begin{array}{l}\text { AIR } \\
\text { CLAS }\end{array}$ & $\begin{array}{l}\text { AIR } \\
\text { MOL }\end{array}$ & MU & $\begin{array}{l}3000 * \\
\text { LOG }\end{array}$ \\
\hline PRCT & $\begin{array}{l}\text { DEG } \\
\text { CENT }\end{array}$ & CPS 1 & METRS & $\begin{array}{l}\text { WALL } \\
D B / S \\
\end{array}$ & $\begin{array}{l}\text { WALL } \\
\mathrm{DB} / \mathrm{S}\end{array}$ & $D B / S$ & $/ M$ & IM & $/ M$ & & $\mathrm{RH}$ \\
\hline
\end{tabular}

12345678901234567890123456789012345678901234567890123456789012345678

$\begin{array}{llll}.1 & 0 & 132 & 2.520 \\ 3.1 & 0 & 132 & 2.520 \\ 4.1 & 0 & 132 & 2.520 \\ 4.4 & 0 & 132 & 2.520 \\ 5.4 & 0 & 132 & 2.520 \\ 6.2 & 0 & 132 & 2.520 \\ 7.3 & 0 & 132 & 2.520 \\ 8.9 & 0 & 132 & 2.520 \\ 11.1 & 0 & 132 & 2.520 \\ 11.9 & 0 & 132 & 2.520 \\ 12.4 & 0 & 132 & 2.520 \\ 13.0 & 0 & 132 & 2.520 \\ 15.4 & 0 & 132 & 2.520 \\ 17.5 & 0 & 132 & 2.520 \\ 25.1 & 0 & 132 & 2.520 \\ 27.4 & 0 & 132 & 2.520 \\ 33.6 & 0 & 132 & 2.520 \\ 38.4 & 0 & 132 & 2.520 \\ 44.6 & 0 & 132 & 2.520 \\ 54.0 & 0 & 132 & 2.520 \\ 64.0 & 0 & 132 & 2.520 \\ 84.0 & 0 & 132 & 2.520 \\ 91.0 & 0 & 132 & 2.520\end{array}$

$\begin{array}{llllllll}2.10 & 1.88 & .22 & 0015 & 0007 & 0015 & 0038-2999 \\ 2.60 & 1.88 & .72 & 0050 & 0007 & 0050 & 0126 & 1474 \\ 2.67 & 1.88 & .79 & 0055 & 0007 & 0055 & 0138 & 1838 \\ 2.67 & 1.88 & .79 & 0055 & 0007 & 0055 & 0138 & 1930 \\ 2.64 & 1.88 & .76 & 0053 & 0007 & 0053 & 0133 & 2197 \\ 2.53 & 1.88 & .65 & 0045 & 0007 & 0045 & 0113 & 2377 \\ 2.55 & 1.88 & .67 & 0047 & 0007 & 0047 & 0118 & 2590 \\ 2.34 & 1.88 & .46 & 0032 & 0007 & 0032 & 0080 & 2848 \\ 2.37 & 1.88 & .49 & 0034 & 0007 & 0034 & 0085 & 3136 \\ 2.26 & 1.88 & .38 & 0026 & 0007 & 0026 & 0065 & 3227 \\ 2.17 & 1.88 & .29 & 0020 & 0007 & 0020 & 0050 & 3280 \\ 2.10 & 1.88 & .22 & 0015 & 0007 & 0015 & 0038 & 3342 \\ 2.10 & 1.88 & .22 & 0015 & 0007 & 0015 & 0038 & 3563 \\ 2.07 & 1.88 & .19 & 0013 & 0007 & 0013 & 0033 & 3729 \\ 2.04 & 1.88 & .16 & 0011 & 0007 & 0011 & 0028 & 4199 \\ 2.02 & 1.88 & .14 & 0010 & 0007 & 0010 & 0025 & 4313 \\ 2.02 & 1.88 & .14 & 0010 & 0007 & 0010 & 0025 & 4579 \\ 1.97 & 1.88 & .09 & 0006 & 0007 & 0006 & 0015 & 4753 \\ 2.02 & 1.88 & .14 & 0010 & 0007 & 0010 & 0025 & 4948 \\ 1.99 & 1.88 & .11 & 0008 & 0007 & 0008 & 0020 & 5197 \\ 2.03 & 1.88 & .15 & 0010 & 0007 & 0010 & 0025 & 5419 \\ 1.99 & 1.88 & .11 & 0008 & 0007 & 0008 & 0020 & 5773 \\ 2.00 & 1.88 & .12 & 0008 & 0007 & 0008 & 0020 & 5877\end{array}$

$\begin{array}{rlll}.1 & 0 & 282 & 1.170 \\ 3.1 & 0 & 282 & 1.170 \\ 4.1 & 0 & 282 & 1.170 \\ 4.4 & 0 & 282 & 1.170 \\ 5.4 & 0 & 282 & 1.170 \\ 6.2 & 0 & 282 & 1.170 \\ 7.3 & 0 & 282 & 1.170 \\ 8.9 & 0 & 282 & 1.170 \\ 11.1 & 0 & 282 & 1.170 \\ 11.9 & 0 & 282 & 1.170 \\ 12.4 & 0 & 282 & 1.170 \\ 13.0 & 0 & 282 & 1.170 \\ 15.4 & 0 & 282 & 1.170 \\ 17.5 & 0 & 282 & 1.170 \\ 25.1 & 0 & 282 & 1.170 \\ 27.4 & 0 & 282 & 1.170 \\ 33.6 & 0 & 282 & 1.170 \\ 38.4 & 0 & 282 & 1.170 \\ 44.6 & 0 & 282 & 1.170 \\ 54.0 & 0 & 282 & 1.170 \\ 64.0 & 0 & 282 & 1.170 \\ 84.0 & 0 & 282 & 1.170 \\ 91.0 & 0 & 282 & 1.170\end{array}$

$\begin{array}{llllllll}1.63 & 1.34 & .29 & 0020 & 0031 & 0020 & 0024-2999\end{array}$ $2.491 .34 \quad 1.150080 \quad 00310080 \quad 0094 \quad 1474$ $2.75 \quad 1.34 \quad 1.410098 \quad 00310098 \quad 0115 \quad 1838$ $2.92 \quad 1.34 \quad 1.58 \quad 0110 \quad 003101100129 \quad 1930$ $3.051 .34 \quad 1.71 \quad 0119003101190140 \quad 2197$ $\begin{array}{llllllll}3.08 & 1.34 & 1.74 & 0121 & 0031 & 0121 & 0142 & 2377\end{array}$ $\begin{array}{lllllllll}3.14 & 1.34 & 1.80 & 0125 & 0031 & 0125 & 0147 & 2590\end{array}$ $\begin{array}{lllllllll}3.07 & 1.34 & 1.73 & 0120 & 0031 & 0120 & 0141 & 2848\end{array}$ $\begin{array}{llllllll}2.95 & 1.34 & 1.61 & 0112 & 0031 & 0112 & 0132 & 3136\end{array}$ $\begin{array}{llllllll}2.75 & 1.34 & 1.41 & 0098 & 0031 & 0098 & 0115 & 3227\end{array}$ $2.58 \quad 1.34 \quad 1.24 \quad 0086 \quad 00310086 \quad 0101 \quad 3280$ $2.391 .34 \quad 1.05 \quad 0073 \quad 00310073 \quad 0086 \quad 3342$ $\begin{array}{llllllll}2.22 & 1.34 & .88 & 0061 & 0031 & 0061 & 0072 & 3563\end{array}$ $2.06 \quad 1.34 \quad .720050 \quad 00310050 \quad 00593729$ $\begin{array}{llllllll}1.88 & 1.34 & .54 & 0038 & 0031 & 0038 & 0045 & 4199\end{array}$ $\begin{array}{lllllllll}1.87 & 1.34 & .53 & 0037 & 0031 & 0037 & 0043 & 4313\end{array}$ $\begin{array}{llllllll}1.74 & 1.34 & .40 & 0028 & 0031 & 0028 & 0033 & 4579\end{array}$ $\begin{array}{llllllll}1.69 & 1.34 & .35 & 0024 & 0031 & 0024 & 0028 & 4753\end{array}$ $\begin{array}{lllllllll}1.65 & 1.34 & .31 & 0022 & 0031 & 0022 & 0026 & 4948\end{array}$ $\begin{array}{llllllll}1.64 & 1.34 & .30 & 0021 & 0031 & 0021 & 0025 & 5197\end{array}$

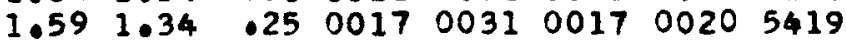
$\begin{array}{llllllll}1.59 & 1.34 & .25 & 0017 & 0031 & 0017 & 0020 & 5773\end{array}$ $\begin{array}{llllllll}1.59 & 1.34 & .25 & 0017 & 0031 & 0017 & 0020 & 5877\end{array}$

* last column employed in computer plotting only. 
TABLE NO. 2

TABULATION OF ORIGINAL DATA POINTS FOR

ABSORPTION OF S@UND IN AIR VERSUS RELATIVE HUMIDITY AT O DEGREES CENTIGRADE

\begin{tabular}{|c|c|c|c|c|c|c|c|c|c|c|c|}
\hline $\mathrm{R} \bullet \mathrm{H} \bullet$ & TEMP & FREQ & LAMDA & $\begin{array}{c}\text { A I R } \\
+\end{array}$ & & $\begin{array}{l}\text { AIR } \\
\text { ONLY }\end{array}$ & $\begin{array}{l}\text { AIR } \\
\text { ONLY }\end{array}$ & $\begin{array}{l}\text { AIR } \\
\text { CLAS }\end{array}$ & $\begin{array}{c}\text { AIR } \\
M O L\end{array}$ & MU & $\begin{array}{l}3000 \\
\text { LOG }\end{array}$ \\
\hline & DEG & CPS & ME TR & $\begin{array}{l}\text { WALL } \\
\text { DB/S }\end{array}$ & $\begin{array}{l}\text { WALL } \\
D B / S\end{array}$ & $08 / 5$ & $/ M$ & $/ M$ & $/ M$ & & $\mathrm{RH}$ \\
\hline & 1 & CPS & 2 & 3 & & 4 & $7 M$ & $5^{/ M}$ & $/ M$ & 6 & \\
\hline
\end{tabular}

12345678901234567890123456789012345678901234567890123456789012345678

\begin{tabular}{|c|c|c|c|c|c|c|c|c|c|c|c|}
\hline$\cdot 1$ & 0 & 486 & - 681 & 1.43 & 1.10 & $\begin{array}{r}33 \\
-30\end{array}$ & 0023 & 0090 & 0022 & $\begin{array}{l}0015 \\
0061\end{array}$ & 4 \\
\hline 3. & $\begin{array}{l}0 \\
0\end{array}$ & $\begin{array}{l}486 \\
486\end{array}$ & $\begin{array}{l}31 \\
31\end{array}$ & $\begin{array}{l}2.40 \\
2.76\end{array}$ & $\begin{array}{l}1.10 \\
1.10\end{array}$ & 30 & $\begin{array}{l}0090 \\
0115\end{array}$ & $\begin{array}{l}0090 \\
0090\end{array}$ & $\begin{array}{l}0089 \\
0114\end{array}$ & $\begin{array}{l}0061 \\
0078\end{array}$ & $\begin{array}{l}1474 \\
1838\end{array}$ \\
\hline $4 \cdot 4$ & 0 & 486 & .681 & 2.98 & 1.10 & & 0131 & 0090 & 0130 & 0089 & 1930 \\
\hline 5. & 0 & 486 & & & & & 0 & 0090 & 53 & 0104 & 2197 \\
\hline 6.2 & 0 & 486 & & 4 & 1 & 2.44 & 0170 & 0090 & 59 & 0115 & ? \\
\hline & 0 & 486 & & & & & 0186 & 0090 & & 0126 & 259 \\
\hline 8 . & 0 & 486 & .6 & 33 & 1 & 2.93 & 0204 & 0090 & 03 & 0138 & 2848 \\
\hline .1 & 0 & 486 & .6 & 4.13 & 1. & 3.03 & 0211 & 0090 & 10 & 0143 & 36 \\
\hline - & 0 & 486 & .68 & 12 & & 2 & 0210 & 0090 & 0209 & 0143 & $2<1$ \\
\hline 2.4 & .0 & 486 & .681 & 37 & 0 & 2 & 0200 & 0090 & 0199 & 0136 & 280 \\
\hline & 0 & 486 & .6 & & & & & 0090 & & 0123 & 34 \\
\hline & 0 & 486 & & & & & 0158 & 0090 & 0157 & 0107 & 563 \\
\hline $7 \cdot 5$ & 0 & 486 & .681 & 5 & 1.10 & 1.95 & 0136 & 0090 & 0135 & 0092 & 729 \\
\hline 5.1 & 0 & 486 & .681 & 58 & 1. & 1 & 0103 & 0090 & 0102 & 0070 & 4199 \\
\hline 7.4 & 0 & 486 & $\cdot \epsilon$ & & & & 0102 & 0090 & 0101 & 0069 & 431 \\
\hline & 0 & 486 & & & & & 0074 & 0090 & & 0050 & 457 \\
\hline & 0 & 486 & .6 & 2 & 1. & .90 & 0063 & 0090 & 0062 & 0042 & 4753 \\
\hline & 0 & 48 & & & & & 0054 & 0090 & 0053 & 0036 & 948 \\
\hline . $c$ & 0 & 486 & .6 & & & & 0049 & 0090 & 0048 & 0033 & 197 \\
\hline+.0 & 0 & 486 & $\cdot c$ & & & 7 & 0040 & 0090 & 0039 & 0027 & +1 \\
\hline .0 & 0 & 48 & & & & & 35 & 00 & 34 & 0023 & \\
\hline & 0 & 486 & & & & & 134 & 0090 & 0 & 0023 & \\
\hline
\end{tabular}

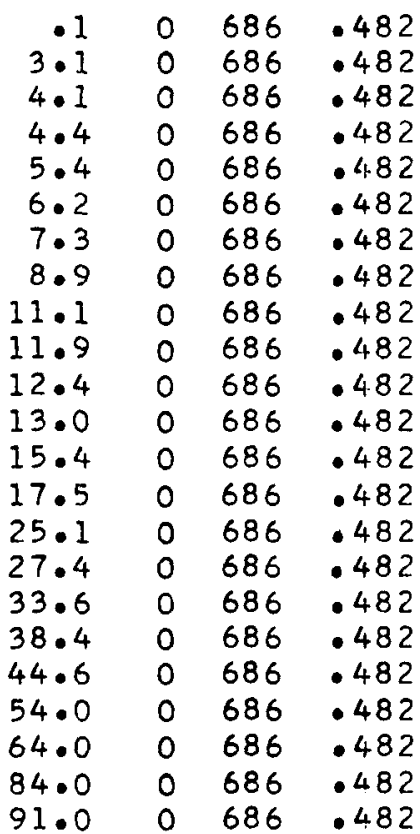

$\begin{array}{llllllll}2.70 & 2.62 & .03 & 0006 & 0179 & 0004 & 0002 & -2999 \\ 3.77 & 2.62 & 1.15 & 0080 & 0179 & 0078 & 0038 & 1474 \\ 4.03 & 2.62 & 1.41 & 0098 & 0179 & 0096 & 0046 & 1838 \\ 4.38 & 2.62 & 1.76 & 0122 & 0179 & 0120 & 0058 & 1930 \\ 4.79 & 2.62 & 2.17 & 0151 & 0179 & 0149 & 0072 & 2197 \\ 5.14 & 2.62 & 2.52 & 0175 & 0179 & 0173 & 0084 & 2377 \\ 5.49 & 2.62 & 2.87 & 0200 & 0179 & 0198 & 0096 & 2590 \\ 5.97 & 2.62 & 3.35 & 0233 & 0179 & 0231 & 0112 & 2848 \\ 6.28 & 2.62 & 3.66 & 0254 & 0179 & 0252 & 0122 & 3136 \\ 6.58 & 2.62 & 3.96 & 0275 & 0179 & 0273 & 0132 & 3227 \\ 6.70 & 2.62 & 4.08 & 0284 & 0179 & 0282 & 0136 & 3280 \\ 6.63 & 2.62 & 4.01 & 0279 & 0179 & 0277 & 0134 & 3342 \\ 6.28 & 2.62 & 3.66 & 0254 & 0179 & 0252 & 0122 & 3563 \\ 5.80 & 2.62 & 3.18 & 0221 & 0179 & 0219 & 0106 & 3729 \\ 5.03 & 2.62 & 2.41 & 0168 & 0179 & 0166 & 0080 & 4199 \\ 5.16 & 2.62 & 2.54 & 0177 & 0179 & 0175 & 0095 & 4313 \\ 4.33 & 2.62 & 1.71 & 0119 & 0179 & 0117 & 0057 & 4579 \\ 4.03 & 2.62 & 1.41 & 0098 & 0179 & 0096 & 0046 & 4753 \\ 3.177 & 2.62 & 1.15 & 0080 & 0179 & 0078 & 0038 & 4948 \\ 3.62 & 2.62 & 1.00 & 0070 & 0179 & 0068 & 0033 & 5197 \\ 3.37 & 2.62 & .75 & 0052 & 0179 & 0050 & 0024 & 5419 \\ 3.26 & 2.62 & .64 & 0044 & 0179 & 0042 & 0020 & 5773 \\ 3.13 & 2.62 & .51 & 0035 & 0179 & 0033 & 0016 & 5877\end{array}$


TABLE NO. 2

TABULATION OF ORIGINAL DATA POINTS FOR

ABSORPTION OF SOUND IN AIR VERSUS RELATIVE HUMIDITY AT O DEGREES CENTIGRADE

\begin{tabular}{|c|c|c|c|c|c|c|c|c|c|c|c|}
\hline$R \bullet H \bullet$ & TEMP & FREQ & LAMDA & $\begin{array}{c}\text { AIR } \\
+\end{array}$ & & $\begin{array}{l}\text { AIR } \\
\text { ONLY }\end{array}$ & $\begin{array}{l}\text { AIR } \\
\text { ONLY }\end{array}$ & $\begin{array}{l}\text { AIR } \\
\text { CLAS }\end{array}$ & $\begin{array}{c}\text { AIR } \\
\text { MOL }\end{array}$ & MU & $\begin{array}{l}3000 * \\
\text { LOG }\end{array}$ \\
\hline PRC & $\begin{array}{l}\text { DEG } \\
\text { CENT } \\
1\end{array}$ & CPS & $\begin{array}{c}\text { ME TRS } \\
2\end{array}$ & $\begin{array}{l}\text { WALL } \\
D B / S \\
3\end{array}$ & $\begin{array}{l}\text { WALL } \\
D B / S\end{array}$ & $\begin{array}{l}D B / S \\
4\end{array}$ & $/ M$ & $5^{/ M}$ & $/ M$ & 6 & RH \\
\hline
\end{tabular}

12345678901234567890123456789012345678901234567890123456789012345678

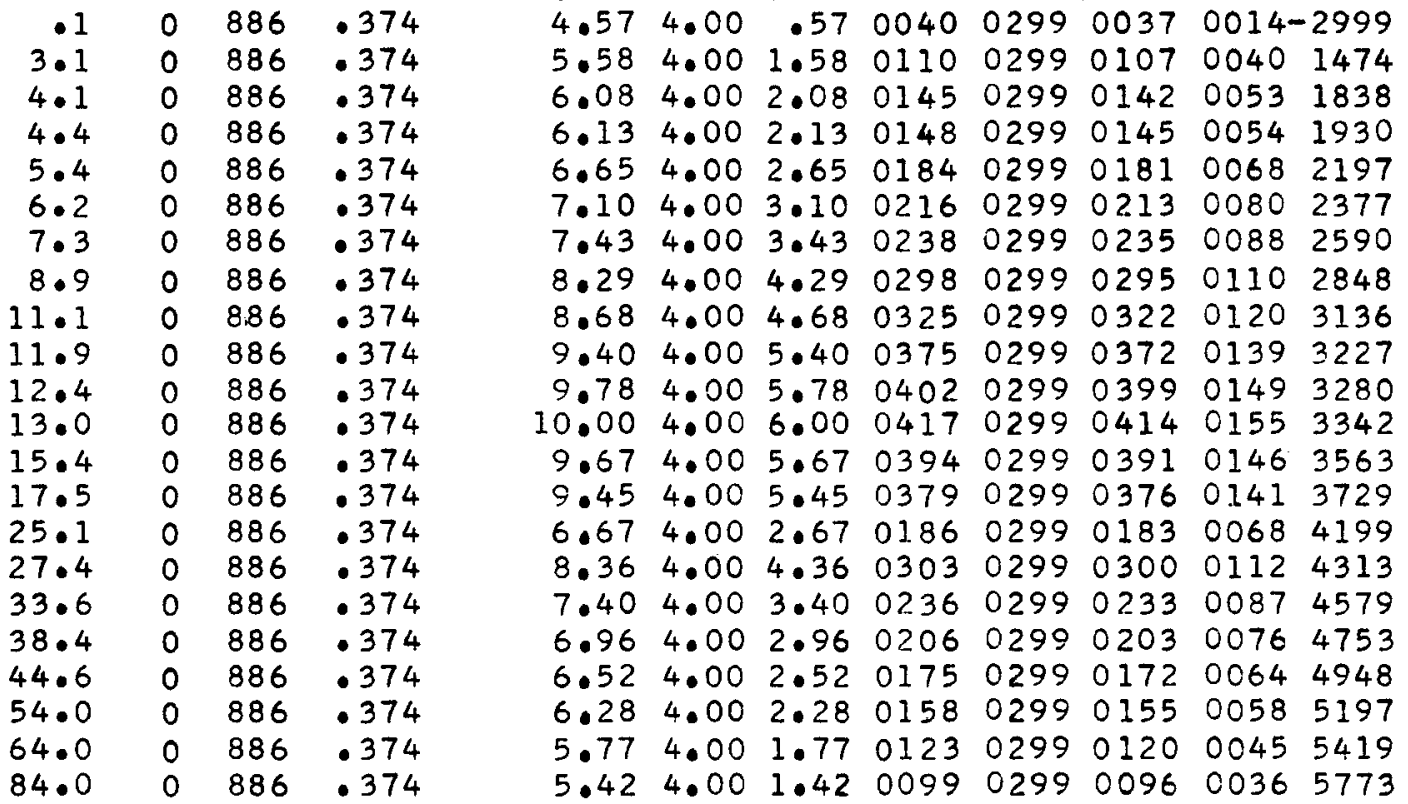

* last column employed in computer plotting only. 
TABLE 3

TABULATION OF ORIGINAL DATA POINTS FOR

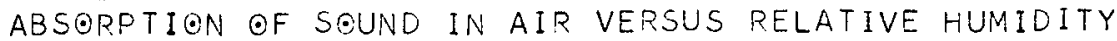
AT -20 DEGREES CENTIGRADE

\begin{tabular}{|c|c|c|c|c|c|c|c|c|c|c|c|}
\hline $\mathrm{R} \bullet \mathrm{H}_{\bullet}$ & & FREQ & LAMDA & $\begin{array}{c}\text { AIR } \\
+\end{array}$ & & $\begin{array}{l}\text { AIR } \\
\text { ONLY }\end{array}$ & $\begin{array}{l}\text { AIR } \\
\text { ONLY }\end{array}$ & $\begin{array}{l}\text { AIR } \\
\text { CLAS }\end{array}$ & $\begin{array}{l}\text { AIR } \\
M \odot L\end{array}$ & MU & $\begin{array}{l}3000 * \\
\text { LOG }\end{array}$ \\
\hline PRCT & $\begin{array}{l}\text { DEG } \\
\text { CENT } \\
1\end{array}$ & CPS & $\begin{array}{c}\text { METRS } \\
2\end{array}$ & $\begin{array}{l}\text { WALL } \\
D R / S \\
3\end{array}$ & $\begin{array}{l}\text { WALL } \\
D R / S\end{array}$ & $\begin{array}{l}D B / S \\
4\end{array}$ & $/ M$ & $5^{/ M}$ & $1 M$ & 6 & $\mathrm{RH}$ \\
\hline
\end{tabular}

12345678901234567890123456789012345678901234567890123456789012345678

\begin{tabular}{|c|c|c|c|}
\hline .0 & -20 & 272 & 1.170 \\
\hline 17.7 & & & \\
\hline 22.2 & -20 & 272 & 1.3 .70 \\
\hline 29.3 & -20 & 272 & 170 \\
\hline $31 \cdot 1$ & -20 & 272 & 170 \\
\hline 37.0 & -20 & 272 & 3.70 \\
\hline 42.8 & -20 & 272 & 170 \\
\hline 46.7 & -2.0 & 272 & 1.170 \\
\hline 58.0 & -20 & 272 & .77 \\
\hline 66.5 & -20 & 272 & 1.170 \\
\hline 72.0 & -20 & 272 & 170 \\
\hline 78.0 & -20 & 272 & 170 \\
\hline 80.0 & -20 & 272 & 170 \\
\hline 98.0 & -20 & 272 & 170 \\
\hline 1.0 & -20 & 468 & . 68,0 \\
\hline 17.7 & -20 & 468 & 80 \\
\hline 2.2 & -20 & 468 & .680 \\
\hline 29.3 & -20 & 468 & .680 \\
\hline $31 \cdot 1$ & -20 & 468 & .680 \\
\hline 37.0 & -20 & 468 & .680 \\
\hline 42.8 & -20 & 468 & .680 \\
\hline 46.7 & -20 & 468 & 680 \\
\hline 58.0 & -20 & 468 & 680 \\
\hline 66.5 & -20 & 468 & .680 \\
\hline 72.0 & -20 & 468 & .680 \\
\hline 78.0 & -20 & 468 & .680 \\
\hline 80.0 & -20 & 468 & .68 \\
\hline 8 . & -20 & 468 & \\
\hline
\end{tabular}

$\begin{array}{lllllllr}1.40 & 1.21 & .19 & 0014 & 0029 & 0014 & 0016 & 0 \\ 1.65 & 1.21 & .44 & 0032 & 0029 & 0032 & 0038 & 3744 \\ 1.90 & 1.21 & .69 & 0050 & 0029 & 0050 & 0059 & 4039 \\ 2.10 & 1.21 & .89 & 0064 & 0029 & 0064 & 0075 & 4401 \\ 2.16 & 1.21 & .95 & 0069 & 0029 & 0069 & 0081 & 4478 \\ 2.16 & 1.21 & .95 & 0069 & 0029 & 0069 & 0081 & 4705 \\ 2.17 & 1.21 & .91 & 0056 & 0029 & 0066 & 0077 & 4894 \\ 2.05 & 1.21 & .84 & 0061 & 0029 & 0061 & 0072 & 5008 \\ 1.95 & 1.21 & .74 & 0053 & 0029 & 0053 & 0062 & 5290 \\ 1.86 & 1.21 & .65 & 0047 & 0029 & 0047 & 0055 & 5468 \\ 1.79 & 1.21 & .58 & 0042 & 0029 & 0042 & 0049 & 5572 \\ 1.73 & 1.21 & .52 & 0038 & 0029 & 0038 & 0045 & 5676 \\ 1.71 & 1.21 & .50 & 0036 & 0029 & 0036 & 0042 & 5709 \\ 1.68 & 1.21 & .47 & 0034 & 0029 & 0034 & 0040 & 5974 \\ 1.19 & .99 & .20 & 0014 & 0086 & 0013 & 0009 & 0 \\ 1.45 & .99 & .46 & 0033 & 0086 & 0032 & 0022 & 3744 \\ 1.78 & .99 & .79 & 0057 & 0086 & 0056 & 0038 & 4039 \\ 2.04 & .99 & 1.05 & 0076 & 0086 & 0075 & 0051 & 4401 \\ 2.34 & .99 & 1.35 & 0098 & 0086 & 0097 & 0066 & 4478 \\ 2.55 & .99 & 1.56 & 0113 & 0086 & 0112 & 0076 & 4705 \\ 2.63 & .99 & 1.64 & 0118 & 0086 & 0117 & 0080 & 4894 \\ 2.64 & .99 & 1.65 & 0119 & 0086 & 0118 & 0080 & 5008 \\ 2.62 & .99 & 1.63 & 0118 & 0086 & 0117 & 0080 & 5290 \\ 2.53 & .99 & 1.54 & 0111 & 0086 & 01110 & 0075 & 5468 \\ 2.42 & .99 & 1.43 & 0103 & 0086 & 0102 & 0070 & 5572 \\ 2.31 & .99 & 1.32 & 0095 & 0086 & 0094 & 0064 & 5676 \\ 2.25 & .99 & 1.26 & 0091 & 0086 & 0090 & 0061 & 5709 \\ 2.10 & .99 & 1.11 & 0080 & 0086 & 0079 & 0054 & 5974\end{array}$

* last column employed in computer plotting only. 
TARLF 3

TAOULATION OF OPIGIMIAL DATA POINTS FOR

APSOPPTION OF SOUND IN AIR VERSUS RELATIVE. HUMIDITY AT -20 DEGRFES CFNTIGRADF

\begin{tabular}{|c|c|c|c|c|c|c|c|c|c|c|c|c|}
\hline $\mathrm{R} \bullet \mathrm{H}_{\bullet}$ & TEMP & FREQ & LAMDA & & $\begin{array}{c}\text { A I R } \\
+\end{array}$ & & $\begin{array}{l}\text { AIR } \\
\text { ONLY }\end{array}$ & $\begin{array}{l}\text { AIR } \\
\text { ONLY }\end{array}$ & $\begin{array}{l}\text { AIR } \\
\text { CLAS }\end{array}$ & $\begin{array}{l}\text { AIR } \\
M O L\end{array}$ & $M U$ & 3000 \\
\hline PR.CT & $\begin{array}{l}\text { DEG } \\
C E N T \\
I\end{array}$ & CPS & $\begin{array}{c}M E T R S \\
2\end{array}$ & $'$ & $\begin{array}{l}\text { WALL } \\
\text { DP, /S } \\
3\end{array}$ & $\begin{array}{l}\text { WALL } \\
D B / S\end{array}$ & $\begin{array}{l}D B / .5 \\
4\end{array}$ & $M$ & $/ M$ & $/ M$ & & $\mathrm{RH}$ \\
\hline
\end{tabular}
12345678901234567890123456789012345678901234567890123456789012345678

\begin{tabular}{|c|c|c|c|c|c|c|c|c|c|c|c|}
\hline 1.0 & -20 & 660 & . 482 & 2.00 & 1.86 & .14 & 0010 & 0170 & 0008 & 0004 & 0 \\
\hline 17.7 & -20 & 660 & .482 & 2.32 & 1.86 & .46 & 0033 & 0170 & 0031 & 0015 & 3744 \\
\hline 22.2 & -20 & 660 & .482 & 2.65 & $1 \cdot 86$ & .80 & 0058 & 0170 & 0056 & 0027 & 4039 \\
\hline $29 \cdot 3$ & -20 & 660 & .482 & 2.98 & 1.86 & 1.12 & 0081 & 0170 & 0079 & 0038 & 4401 \\
\hline $31 \cdot 1$ & -20 & 660 & .482 & 3.40 & 1.86 & 1.54 & 0111 & 0170 & 0109 & 0053 & 4478 \\
\hline 7.0 & -20 & 660 & .482 & 3.73 & 1.86 & 1.87 & 0135 & 0170 & 0133 & 0064 & 4705 \\
\hline $42 \cdot 8$ & -20 & 660 & .482 & 3.99 & $1 \cdot 86$ & 2.13 & 0154 & 0170 & 0152 & 0073 & 4894 \\
\hline 46.7 & -20 & 660 & .432 & 4.14 & 1.86 & 2.28 & 0165 & 0170 & 0163 & 0079 & 5008 \\
\hline 58.0 & -20 & 660 & . 482 & 4.25 & 1.86 & 2.39 & 0173 & 01.70 & 0171 & 0033 & 5290 \\
\hline 66.5 & -20 & 660 & - 482 & 4.23 & 1.86 & 2.37 & 0171 & 0170 & 0169 & 0082 & 5468 \\
\hline 72.0 & -20 & 660 & .482 & 4.20 & 1. .86 & 2.34 & 0169 & 0170 & 0167 & 0081 & 5572 \\
\hline 78.0 & -20 & 660 & $.48 ?$ & 4.08 & 9.86 & 2.22 & 0160 & 0170 & 0158 & 0076 & 5676 \\
\hline 80.0 & -20 & 660 & - 482 & 4.04 & 1.86 & 2.18 & 0157 & 0170 & 0155 & 0075 & 5709 \\
\hline 98.0 & -20 & 660 & .482 & 3.96 & 1.86 & 2.10 & 0152 & 0170 & 0150 & 0072 & 5974 \\
\hline 1.0 & -20 & 853 & .373 & 3.62 & 3.58 & .04 & 0003 & 0288 & 0000 & 0000 & 0 \\
\hline 17.7 & -20 & 853 & .373 & 3.82 & 3.58 & .24 & 0017 & 0288 & 0014 & 0005 & 3744 \\
\hline $22 \cdot 2$ & -20 & 853 & .373 & 4.05 & 3.58 & .47 & 0034 & 0288 & 0031 & 0012 & 4039 \\
\hline $29 \cdot 3$ & -20 & 853 & . 373 & $4 \cdot 38$ & & 80 & 0058 & 0288 & 0055 & 0021 & 4401 \\
\hline $31 \cdot 1$ & -20 & 853 & .373 & 4.83 & 3.58 & 1.25 & 0090 & 0288 & 0087 & 0033 & 4478 \\
\hline 37.0 & -20 & 853 & .373 & 5.27 & 3.58 & 1.69 & 0122 & 0288 & 0119 & 0045 & 4705 \\
\hline 42.8 & -20 & 853 & .373 & 5.67 & 3.58 & 2.09 & 0151 & 0288 & 0148 & 0055 & 4894 \\
\hline 45.7 & -2.0 & 853 & .373 & 5.92 & 3.58 & 2.34 & 0169 & 0288 & 0166 & $\cos 2$ & 5008 \\
\hline 58.0 & -20 & 853 & .373 & $6 . ? 0$ & 3.58 & 2.62 & 0199 & 0280 & 0186 & 0070 & 5290 \\
\hline 66.5 & -20 & 853 & .373 & 6.20 & $3 \cdot 58$ & 2.62 & 0189 & 0288 & 0186 & 0070 & 5468 \\
\hline 72.0 & -20 & 853 & .373 & 6.35 & 3.58 & 2.77 & 0200 & 0288 & 0197 & 0074 & 5572 \\
\hline 78.0 & -20 & 853 & .373 & 6.29 & 3.58 & 2.71 & 0196 & 0288 & 0193 & 0072 & 5676 \\
\hline 80.0 & -20 & 853 & .373 & 6.21 & 3.58 & 2.63 & 0190 & 0288 & 0187 & 0070 & 5709 \\
\hline 98.0 & -20 & 853 & .373 & 6.25 & 3.58 & 2.67 & 0193 & 0288 & 0190 & 0071 & 5974 \\
\hline
\end{tabular}

* last column employed in computer plotting only. 
TABLE 4

TABULATION OF ORIGINAL DATA PEINTS FOR

ABSORPTION OF SOUND IN AIR VERSUS RELATIVE HUMIDITY

AT -40 DEGREES CENTIGRADE

R.H. TEMP FREQ LAMDA

AIR AIR AIR AIR AIR MU 3000*

$+\quad$ ONLY ONLY CLAS MOL LOG

DEG

WALL WALL

PRCT CENT CPS METRS

$D E / S \quad D R / S \quad D B / S / M \quad / M \quad / M$

$\begin{array}{lllllll}1 & 2 & 3 & 4 & 5 & 6\end{array}$

12345678901.234567890123456789012345678901234567890123456789012345678

\begin{tabular}{|c|c|c|c|c|c|c|c|c|c|c|c|}
\hline 9.0 & 40 & 260 & 1.170 & 1.34 & 1.31 & .13 & 0010 & 0083 & 0009 & 0007 & 2680 \\
\hline 32.0 & 40 & 260 & 1.170 & 1.33 & 1.31 & .12 & 0009 & 0033 & 0008 & 0006 & 4510 \\
\hline 43.0 & 40 & 260 & 1.170 & 1.37 & $1 \cdot 31$ & .16 & 0012 & 0083 & 0011 & 0008 & 4910 \\
\hline $74 \cdot 0$ & 40 & 260 & 1.170 & 1.39 & $1 \cdot 31$ & .18 & 0013 & 0083 & 0012 & 0009 & 5510 \\
\hline 88.0 & 40 & 260 & 1.170 & 1.38 & $1 \cdot 31$ & .17 & 0013 & 0083 & 0012 & 0009 & 5830 \\
\hline 97.0 & 40 & 260 & 1.170 & 1.40 & 1.31 & .19 & 0014 & 0083 & 0013 & 0010 & 5970 \\
\hline 9.0 & 40 & 447 & .680 & 1.15 & .95 & .20 & 0015 & 0164 & 0013 & 0010 & 2860 \\
\hline $32 \cdot 0$ & 40 & 447 & .680 & 1.12 & .95 & .17 & 0013 & 0164 & 001.1 & 0009 & 4510 \\
\hline 43.0 & 40 & 447 & .680 & 1.14 & .95 & .19 & 0014 & 0164 & 0013 & 0010 & 4910 \\
\hline 74.0 & 40 & 447 & .680 & 1.17 & .95 & .22 & 0016 & 0164 & 0014 & 0011 & 5610 \\
\hline 88.0 & 40 & 447 & .680 & 1.17 & .95 & .23 & 0016 & 0164 & 0014 & 0011 & 5930 \\
\hline 97.0 & 40 & 447 & .680 & 1.18 & .95 & .24 & 0017 & 0164 & 0015 & 0012 & 5970 \\
\hline 9.0 & 40 & 631 & .482 & .82 & 1.71 & .11 & 0008 & 0277 & 0005 & 0004 & 2860 \\
\hline 32.0 & 40 & 631 & .482 & 1.82 & 1.71 & .11 & 0008 & 0277 & 0005 & 0004 & 4510 \\
\hline 43.0 & 40 & 631 & .482 & 1.83 & 1.71 & .12 & 0009 & 0277 & 0005 & 0004 & 4910 \\
\hline $74 \cdot 0$ & 40 & 631 & .482 & 1.87 & 1.71 & .16 & 0012 & 0277 & 0009 & 0006 & 5610 \\
\hline 88.0 & 40 & 631 & .482 & 1.87 & 1.71 & .16 & 0012 & 0277 & 0009 & 0006 & 5830 \\
\hline 97.0 & 40 & 631 & .482 & 1.87 & 1.71 & .16 & 0012 & 0277 & 0009 & 0006 & 5970 \\
\hline
\end{tabular}




\author{
TABLE NO• 5 \\ TABULATION OF DATA POINTS FOR \\ ABSORPTION OF SOUND IN DRY NITROGEN \\ AT 20 DEGREES CENTIGRADE \\ FOR THE COMPUTATION OF WALL LOSSES
}

\begin{tabular}{|c|c|c|c|c|c|c|c|c|}
\hline MICROPHN & 1111 & 111111 & 2222 & 2222222 & 2222 & 2222222 & 111 & 1111111 \\
\hline TEMP & $+20 \mathrm{C}$ & & $O C$ & & $-20 c$ & & -40 & \\
\hline MODE & $F$ & $\mathbf{R}$ & $F$ & $\mathbf{R}$ & $\mathbf{F}$ & $\mathbf{R}$ & $F$ & $\mathbf{R}$ \\
\hline 11 & 139 & $2 \cdot 14$ & 134 & $1 \cdot 88$ & 129 & 1.92 & - & $-\infty$ \\
\hline 01 & 298 & 1.39 & 288 & $1 \cdot 34$ & 277 & 1.28 & 265 & 1.21 \\
\hline 02 & 512 & 1.20 & 495 & $1 \cdot 10$ & 475 & 1.02 & 456 & 0.95 \\
\hline 03 & 724 & 2.06 & 698 & $2 \cdot 62$ & 673 & 1.93 & 644 & 1.71 \\
\hline 04 & 933 & 4.31 & 900 & 4.00 & 867 & 3.49 & 830 & 3.13 \\
\hline
\end{tabular}


TABLE 6A----400 MIM PRESSURF

TABULATION OF ORIGINAL DATA POINTS FOR

ABSORPTION OF SOUNO IN AIR VERSUS RELATIVE HUMIDITY

AT 20 DEGREES CENTIGRADE

\begin{tabular}{|c|c|c|c|c|c|c|c|c|c|c|c|}
\hline $\mathrm{R} \bullet \mathrm{H} \bullet$ & TEMP & $F R E Q$ & $\checkmark$ LAMLA & $\begin{array}{c}\text { AIR } \\
+\end{array}$ & & AIR & AIR & $A I R$ & $A I R$ & MU & $3000 \%$ \\
\hline PRCT & $\begin{array}{l}\text { DEG } \\
\text { CENT }\end{array}$ & CDS & METRS & $\begin{array}{l}\text { WALL } \\
D B / S\end{array}$ & $\begin{array}{l}\text { WALL } \\
D E / S\end{array}$ & $D B / S$ & $/ M$ & $/ M$ & liM & & $\begin{array}{l}\text { LOG } \\
\text { RH }\end{array}$ \\
\hline
\end{tabular}

\begin{tabular}{|c|c|c|c|c|c|c|c|c|c|c|c|c|}
\hline 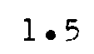 & 0 & 36 & & 00 & 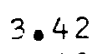 & 5 & 0 & & & & & \\
\hline & & 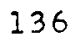 & & 0 & & 42 & .73 & 0052 & 0013 & & & \\
\hline & 20 & & & 400 & 11 & $?$ & .69 & 46 & 13 & +6 & 6 & \\
\hline 2. & 20 & 36 & & 400 & & 42 & .54 & 36 & 0013 & 0036 & & 0 \\
\hline & & & & 00 & & & .38 & & & & 6 & \\
\hline & 20 & & & 400 & & & .33 & & & 22 & & \\
\hline 4 . & ? & & & 40 & & & & & & & & \\
\hline & & & & & & & & & & & & \\
\hline & 20 & & & 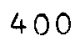 & & & & & & & 0 & \\
\hline & 2 & 36 & & 40 & & & & & & & & \\
\hline & & & & & & & & & & & & \\
\hline & $<$ & & & & & & & & & & & \\
\hline & 20 & & & 40 & & & 2 & & & & & \\
\hline & 20 & 36 & & 40 & & 2. & .18 & & & & 0 & \\
\hline & 20 & 36 & & 40 & & & .2 & & & & & \\
\hline & 20 & & & 40 & & & ? & & & & & \\
\hline & 20 & & & 40 & & & $? ?$ & & & & & \\
\hline & 20 & & & 40 & & & 18 & & & & 10 & 455 \\
\hline & 20 & & & & & & & & & & 30 & \\
\hline & & & & & & & & & & & & \\
\hline & & & & 0 & 29 & & .54 & & & & & 76 \\
\hline & 20 & & & & & & & & & & & \\
\hline & 20 & & & & & & & & & & & 0 \\
\hline & 20 & 9 & & 40 & & & 1.65 & & & & & 102 \\
\hline & 20 & 3 & & 40 & & & .32 & & & & & 134. \\
\hline & 20 & 93 & & 40 & 6 & & 1.11 & & & & 87 & 04 \\
\hline & 20 & 29 & & 40 & & & 9 & & & & & \\
\hline & 20 & & & & & & & & & & & \\
\hline & 20 & 9 & & & & & - 62 & & & & 48 & 24 \\
\hline & $?$ & 29 & & & & & 54 & & & & 41 & 70 \\
\hline & 20 & 9 & & & & & 47 & & & & & \\
\hline & 20 & 29 & & & & & $04 t$ & & & & 34 & \\
\hline & 20 & 19 & & 40 & & & .4 & & & & 0034 & 341 \\
\hline & 20 & 293 & & 40 & & & & & & & 0 & \\
\hline & 20 & 10 & & 400 & & & .4 & & & & & \\
\hline & 20 & & & & & & .5 & & & & 0039 & \\
\hline & 2 & 93 & & 40 & & & & & & & 039 & 455 \\
\hline & & 7 & & 400 & & & & & & & & 41 \\
\hline
\end{tabular}

* last column employed in computer plotting only. 
TABLE 6A- - - 400 MM PRESSURE

TABULATION OF ORIGINAL DATA POINTS FOR

ABSORPTION OF SOUND IN AIR VERSUS RELATIVE HUMIDITY

AT 20 DEGREES CENTIGRADE

\begin{tabular}{|c|c|c|c|c|c|c|c|c|c|c|c|}
\hline $\mathrm{R} \bullet \mathrm{H} \bullet$ & TEMP & FREQ & LAMDA & $\begin{array}{c}\text { AIR } \\
+\end{array}$ & & $\begin{array}{l}\text { AIR } \\
\text { ONLY }\end{array}$ & $\begin{array}{l}\text { AIR } \\
\text { ONLY }\end{array}$ & $\begin{array}{l}\text { AIR } \\
\text { CLAS }\end{array}$ & $\begin{array}{l}A I R \\
M \odot L\end{array}$ & MU & $\begin{array}{l}3000 * \\
\text { LOG }\end{array}$ \\
\hline PRCT & $\begin{array}{l}\text { DEG } \\
\text { CENT }\end{array}$ & CPS & METRS & $\begin{array}{l}\text { WALL } \\
\mathrm{DB} / \mathrm{S}\end{array}$ & $\begin{array}{l}\text { WALL } \\
D B / S\end{array}$ & $D B / S$ & $/ M$ & $/ M$ & $/ M^{\circ}$ & & $\mathrm{RH}$ \\
\hline
\end{tabular}

12345678901234567890123456789012345678901234567890123456789012345678

\begin{tabular}{|c|c|c|c|c|c|c|c|c|c|c|c|c|}
\hline $\begin{array}{l}1.5 \\
1.8\end{array}$ & $\begin{array}{l}20 \\
20\end{array}$ & $\begin{array}{l}04 \\
04\end{array}$ & $\begin{array}{r}.681 \\
.681\end{array}$ & $\begin{array}{l}400 \\
400\end{array}$ & $\begin{array}{l}5.52 \\
6.33\end{array}$ & $\begin{array}{l}1.68 \\
1.68\end{array}$ & $\begin{array}{l}3.84 \\
4.65\end{array}$ & $\begin{array}{l}0258 \\
0312\end{array}$ & $\begin{array}{l}0180 \\
0180\end{array}$ & $\begin{array}{l}0256 \\
0310\end{array}$ & $\begin{array}{l}0174 \\
0211\end{array}$ & $\begin{array}{l}528 \\
766\end{array}$ \\
\hline 1.5 & 20 & 04 & 81 & 400 & 42 & 68 & $4 \cdot 74$ & 0318 & 0180 & 0316 & 0215 & 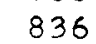 \\
\hline $2 \cdot$ & 20 & 04 & 81 & 400 & 23 & 68 & 4.55 & 05 & 80 & & 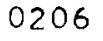 & \\
\hline $2 \cdot 2$ & 20 & 04 & 681 & 400 & & 8 & 4.08 & 0274 & 180 & 272 & 185 & 102 \\
\hline $2 \cdot 8$ & 20 & 04 & & 400 & & & 3.49 & 234 & 80 & 32 & & 34 \\
\hline 4. & 20 & 504 & & 400 & 4.65 & & 97 & 0199 & & & & \\
\hline 5. & 20 & 504 & & 400 & & & & & & & & \\
\hline 5. & 20 & 504 & & 400 & & & 1.98 & 33 & & & & 29 \\
\hline 6.7 & 20 & 504 & & 40 & & & & & & & & 247 \\
\hline 8.0 & 2 & 0 & - & 40 & & & 1.26 & & & & & 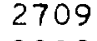 \\
\hline - & 2 & 04 & & 40 & & & 1.00 & & & 0065 & 044 & 30 \\
\hline & 20 & 04 & & 40 & & & .87 & & & & & 325 \\
\hline 8 & 20 & 504 & & & & & 83 & & & & & \\
\hline 3 & 20 & 504 & $\cdot$ & 40 & . 48 & 1.68 & . 80 & 4 & & 0 & 35 & 3802 \\
\hline & 20 & 04 & & 40 & & & .77 & $2<$ & & 0050 & 34 & 40 \\
\hline$\bullet$ & 20 & 504 & & 40 & & & .79 & & & & & $42\}$ \\
\hline & 20 & 504 & & 40 & & & .82 & & & & 6 & \\
\hline . & 20 & 0 & & & & & .83 & & & & & \\
\hline $1 \cdot$ & & 12 & & 40 & & & .38 & & & & 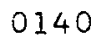 & 52 \\
\hline 1.8 & 2 & 712 & .482 & 400 & & & 5.98 & 0401 & & 03 & 91 & 76 \\
\hline 1. & 20 & 712 & .482 & 0 & 38 & & 6.46 & 0434 & 0 & 0430 & 207 & 836 \\
\hline $2 \cdot c$ & 20 & 712 & .482 & 40 & 0 & & 6.78 & 0455 & 8 & 0451 & 17 & 90 \\
\hline $2 \cdot 2$ & 20 & 712 & .4 & 40 & & & 6 & 0 & & 04 & & 02 \\
\hline 2. & 20 & 712 & .48 & 40 & & & & & & & & 1 \\
\hline $4 \cdot 8$ & 20 & 12 & .482 & 40 & & & & 0362 & 0 & 0358 & 73 & 204 \\
\hline 5.2 & 20 & 712 & .482 & 40 & 1 & & 4.59 & 0308 & 03 & 0304 & 0147 & 2148 \\
\hline .8 & 20 & 712 & .482 & 40 & 75 & & 3.83 & 02 & 0 & 0253 & 22 & 2 \\
\hline .7 & 20 & 712 & .48 & 40 & & & 3.08 & & & 02 & & 247 \\
\hline 8 & 20 & 71 & 48 & 40 & & & & & & & & 2709 \\
\hline 0.0 & 20 & 712 & 482 & 40 & 4.80 & & 1.88 & 0126 & 0 & 0122 & 0059 & 300 \\
\hline & 20 & 712 & .48 & 40 & 2 & & 1.60 & 0107 & 03 & 0103 & 0050 & 325 \\
\hline & 20 & 712 & 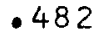 & 40 & & & .46 & 0098 & & & & \\
\hline & 20 & 1 & 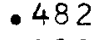 & 40 & 2 & & .29 & & & & 0040 & 380 \\
\hline & 20 & 712 & .48 & 40 & +.08 & & 1.16 & 0078 & & 0074 & 0036 & 4091 \\
\hline & 20 & 712 & .4 & 40 & .07 & & 10 & 0077 & 0368 & 0073 & 0035 & 428 \\
\hline & 20 & 12 & & 40 & & & .12 & 0075 & 03 & 0071 & 0034 & \\
\hline & & 12 & & 0 & & & 15 & 077 & 68 & 1073 & 035 & \\
\hline
\end{tabular}

* last column employed in computer plotting only. 
TABLE GA-m-400 MM PRESSURE

TABULATION OF ORIGINAL DATA POINTS FOR

ABSORPTION OF SOUND IN AIR VERSUS RELATIVE HUMIDITY AT 20 DEGREES CENTIGRADF

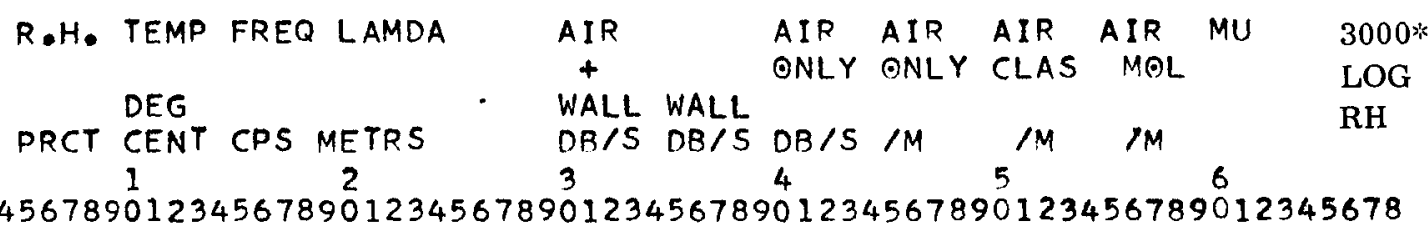

$\begin{array}{rrrllllllllll}1.5 & 20 & 918 & .374 & 400 & 11.00 & 6.98 & 4.02 & 0270 & 0618 & 0264 & 0099 & 528 \\ 1.8 & 20 & 918 & .374 & 400 & 13.00 & 6.98 & 6.02 & 0404 & 0618 & 0398 & 0149 & 766 \\ 1.9 & 20 & 918 & .374 & 400 & 13.00 & 6.98 & 6.62 & 0444 & 0618 & 0438 & 0164 & 836 \\ 2.0 & 20 & 918 & .374 & 400 & 14.70 & 6.98 & 7.72 & 0518 & 0618 & 0512 & 0191 & 903 \\ 2.2 & 20 & 918 & .374 & 400 & 14.70 & 6.98 & 7.72 & 0518 & 0618 & 0512 & 0191 & 1027 \\ 2.8 & 20 & 918 & .374 & 400 & 14.70 & 6.98 & 7.72 & 0518 & 0618 & 0512 & 0191 & 1341 \\ 4.8 & 20 & 918 & .374 & 400 & 14.10 & 6.98 & 7.12 & 0478 & 0618 & 0472 & 0176 & 2044 \\ 5.2 & 20 & 918 & .374 & 400 & 13.30 & 6.98 & 6.32 & 0424 & 0618 & 0418 & 0156 & 2148 \\ 5.8 & 20 & 918 & .374 & 400 & 12.10 & 6.98 & 5.12 & 0344 & 0618 & 0338 & 0126 & 2290 \\ 6.7 & 20 & 918 & .374 & 400 & 11.20 & 6.98 & 4.22 & 0283 & 0618 & 0277 & 0104 & 2478 \\ 8.0 & 20 & 918 & .374 & 400 & 10.20 & 6.98 & 3.22 & 0216 & 0618 & 0210 & 0079 & 2709 \\ 10.0 & 20 & 918 & .374 & 400 & 9.20 & 6.98 & 2.22 & 0149 & 0618 & 0143 & 0053 & 3000 \\ 12.2 & 20 & 918 & .374 & 400 & 8.79 & 6.98 & 1.81 & 0122 & 0618 & 0116 & 0043 & 3259 \\ 13.7 & 20 & 918 & .374 & 400 & 8.79 & 6.98 & 1.81 & 0122 & 0618 & 0116 & 0043 & 3410 \\ 18.5 & 20 & 918 & .374 & 400 & 8.53 & 6.98 & 1.55 & 0104 & 0618 & 0098 & 0037 & 3802 \\ 23.1 & 20 & 918 & .374 & 400 & 8.30 & 6.98 & 1.32 & 0089 & 0618 & 0083 & 0031 & 4091 \\ 26.8 & 20 & 918 & .374 & 400 & 8.03 & 6.98 & 1.05 & 0070 & 0618 & 0064 & 0024 & 4284 \\ 33.0 & 20 & 918 & .374 & 400 & 8.11 & 6.98 & 1.13 & 0076 & 0618 & 0070 & 0026 & 4556 \\ 37.0 & 20 & 918 & .374 & 400 & 8.11 & 6.98 & 1.13 & 0076 & 0618 & 0070 & 0026 & 4705\end{array}$

* last column employed in computer plotting only. 
TABLE 6E----200 MM PRESSURE

TABULATION OF ORIGINAL DATA POINTS FOR

ABSORPTION OF SOUND IN AIR VERSUS RELATIVE HUMIDITY AT 20 DEGREES CENTIGRADE.

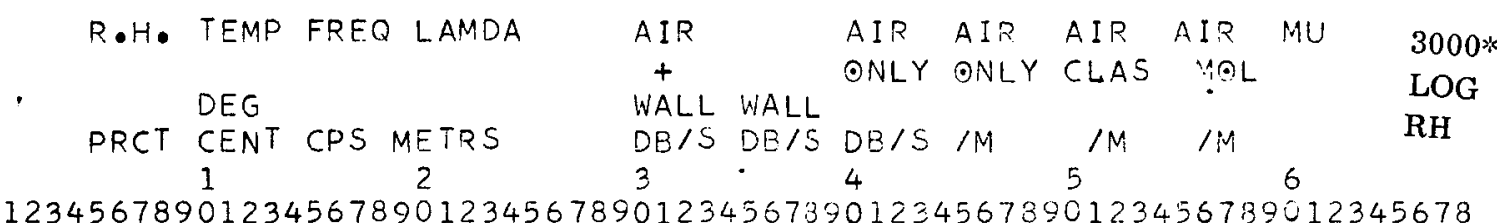

\begin{tabular}{|c|c|c|c|c|}
\hline 1.4 & 20 & 136 & 2.520 & 200 \\
\hline $1 \cdot 5$ & 20 & 135 & 2.520 & 200 \\
\hline 1.7 & 20 & 136 & 2.520 & 200 \\
\hline $1 \cdot 8$ & 20 & 136 & 2.520 & 200 \\
\hline 1.9 & 20 & 136 & 2.520 & 200 \\
\hline 2.1 & 20 & 136 & 2.520 & 200 \\
\hline 2.4 & 20 & 136 & 2.520 & 200 \\
\hline $3 \cdot 1$ & 20 & 136 & 2.520 & 200 \\
\hline $4 \cdot 7$ & 20 & 136 & 2.520 & 200 \\
\hline $6 \cdot 4$ & 20 & 136 & 2.520 & 200. \\
\hline $8 \cdot 3$ & 20 & 136 & 2.520 & 200 \\
\hline 16.7 & 20 & 136 & 2.520 & 200 \\
\hline 22.0 & 20 & 136 & 2.520 & 200 \\
\hline $26 \cdot 3$ & 20 & 136 & 2.520 & 200 \\
\hline 1.4 & 20 & 293 & 1.170 & 200 \\
\hline 1.5 & 20 & 293 & 1.170 & 200 \\
\hline 1.7 & 20 & 293 & 1.170 & 200 \\
\hline $1 \cdot 8$ & 20 & 293 & 170 & 200 \\
\hline $1 \cdot 9$ & 20 & 293 & 1.170 & 200 \\
\hline $2 \cdot 1$ & 20 & 293 & 1.170 & 200 \\
\hline $2 \cdot 4$ & 20 & 293 & 1.170 & 200 \\
\hline - 1 & 20 & 293 & 1.170 & 200 \\
\hline .7 & 20 & 293 & .170 & 200 \\
\hline 6.4 & 20 & 293 & 1.170 & 2.00 \\
\hline .3 & 20 & 293 & 1.170 & 200 \\
\hline 16.7 & 20 & 293 & 1.170 & 200 \\
\hline $22 \cdot 0$ & 20 & 293 & 1.170 & 200 \\
\hline & 20 & 293 & 170 & 0 \\
\hline
\end{tabular}

$\begin{array}{ll}3.52 & 3.05 \\ 3.59 & 3.05 \\ 3.36 & 3.05 \\ 3.30 & 3.05 \\ 3.33 & 3.05 \\ 3.30 & 3.05 \\ 3.20 & 3.05 \\ 3.10 & 3.05 \\ 3.13 & 3.05 \\ 3.05 & 3.05 \\ 3.11 & 3.05 \\ 3.22 & 3.05 \\ 3.23 & 3.05 \\ 3.2 .6 & 3.05 \\ & \\ 4.58 & 2.38 \\ 4.28 & 2.38 \\ 3.78 & 2.38 \\ 3.63 & 2.38 \\ 3.48 & 2.38 \\ 3.31 & 2.38 \\ 3.23 & 2.38 \\ 3.10 & 2.38 \\ 2.95 & 2.38 \\ 2.81 & 2.38 \\ 2.72 & 2.38 \\ 2.74 & 2.38 \\ 2.77 & 2.38 \\ 2.83 & 2.38\end{array}$

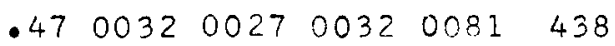

$.540036002700360031 \quad 523$

$.310021002700210053 \quad 691$

$.2500170027 \quad 00170043 \quad 766$

$.280019002700190048 \quad 836$

$.2500170027 \quad 0017 \quad 0043 \quad 967$

$.15001000270010 \quad 00251141$

$.050003002700030008 \quad 1474$

$.080005002700050013 \quad 2016$

$.000000 \quad 0027000000002419$

.0600040027000400102757

$\begin{array}{lllllll}.17 & 0011 & 0027 & 0011 & 0028 & 3668\end{array}$

$\begin{array}{lllllll}.18 & 0012 & 0027 & 00120030 & 4027\end{array}$

. $210014002700140035 \quad 4260$

$\begin{array}{llllllllll}4.58 & 2.38 & 2.20 & 0148 & 0122 & 0147 & 0172 & 438\end{array}$

$\begin{array}{lllllllll}4.28 & 2.38 & 1.90 & 0128 & 0122 & 0127 & 0149 & 528\end{array}$

$\begin{array}{lllllllll}3.78 & 2.38 & 1.40 & 0094 & 0122 & 0093 & 0109 & 591\end{array}$

$\begin{array}{lllllllll}3.63 & 2.38 & 1.25 & 0084 & 0122 & 0083 & 0007 & 766\end{array}$

$\begin{array}{llllllll}3.48 & 2.38 & 1.10 & 0074 & 0122 & 0073 & 0086 & 836\end{array}$

$\begin{array}{lllllllll}3.31 & 2.38 & .93 & 0062 & 0122 & 0061 & 0071 & 967\end{array}$

$\begin{array}{lllllll}.85 & 0057 & 0122 & 0056 & 0066 & 1141\end{array}$

$.720048 \quad 0122 \quad 00470055 \quad 1474$

$.570038012200370043 \quad 2016$

$\begin{array}{llllll}.43 & 0029 & 0122 & 0028 & 0033 & 2419\end{array}$

$\begin{array}{lllllll}.34 & 0023 & 0122 & 0022 & 0026 & 2757\end{array}$

. $360024012200230027 \quad 3668$

. 3900260122002500294027

$.450030012200290034 \quad 4260$

* last column employed in computer plotting only. 
TABLE 6B----200 MM PRESSIUPE

TABULATION OF ORIGINAL DATA POINTS FOR

ABSORPTION OF SOUND IN AIR VERSUS RELATIVE HUMIDITY AT 20 DEGREES CENTIGRADF

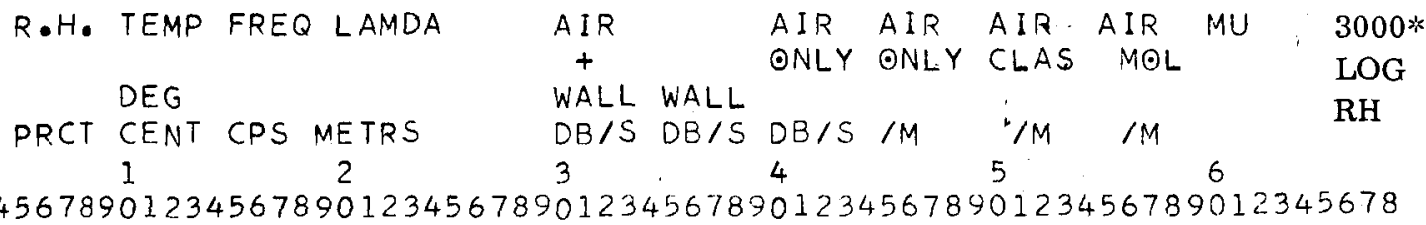

\begin{tabular}{|c|c|c|c|c|c|c|c|c|c|c|c|c|}
\hline 1. & & & & & & 0 & .72 & $\begin{array}{l}7 \\
8\end{array}$ & & & & \\
\hline & 0 & & $i$ & 20 & & & $\begin{array}{l}44 \\
68\end{array}$ & 0247 & & 02 & & \\
\hline & & & & 20 & & & & 0228 & & 24 & & \\
\hline & 20 & & & 20 & & & 0 & 0208 & & 4 & & \\
\hline & & & & 20 & & & & & & & & \\
\hline$c$ & & & & 20 & & & & & & & & \\
\hline & & & & & & & & & & & & \\
\hline & & & & 20 & 10 & 0 & 1. & & & & & \\
\hline & & & & 20 & & & .09 & & & & & \\
\hline & & & & 20 & & & .78 & & & & 3 & \\
\hline & & & & 20 & & & & & & & & \\
\hline & & & & & & & $\theta$ & & & & & \\
\hline & & & & & & & & & & & & \\
\hline & & & & & & & & & & & & \\
\hline & & 71 & 4 & 200 & & & & 40 & & & 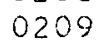 & \\
\hline 1. & 20 & 712 & 482 & 200 & & 14 & & 0407 & 0736 & & 93 & \\
\hline 1. & 20 & 712 & 482 & 200 & & 4 & & 0393 & 36 & & & \\
\hline & 20 & 71 & .4 & 20 & & & & & & & & \\
\hline & 20 & 71 & .48 & 20 & & & & & & & & \\
\hline & 2 & 712 & .482 & 20 & & & 9 & 01 & 36 & & 42 & \\
\hline & 20 & 712 & .482 & 20 & & & & 0257 & 07 & & & \\
\hline & 20 & 71 & 48 & 20 & & & & 0.207 & & & & \\
\hline & 2 & 71 & 4 & 20 & & & & & & & & \\
\hline & 20 & 712 & .482 & 20 & & & 1.44 & 97 & 0736 & & 43 & \\
\hline & 2 & 71 & .48 & 20 & & & & & 0736 & & 027 & \\
\hline & 2 & 71 & 18 & 20 & & & & 0059 & 0736 & & & \\
\hline & & 71 & 4 & & & & & & & & & \\
\hline
\end{tabular}

* last column employed in computer plotting only. 
TABLE 6B- - 200 MM PRESSURE

TABULATION OF ORIGINAL DATA POINTS FOR

ABSORPTI ON OF SOUND IN AIR VERSUS RELATIVE HUMIDITY AT 20 DEGREES CENTIGRADE

\begin{tabular}{|c|c|c|c|c|c|c|c|c|c|c|c|}
\hline $\mathrm{R} \bullet \mathrm{H}$. & TEMP & FREQ & LAMDA & $\begin{array}{c}\text { AIR } \\
+\end{array}$ & & $\begin{array}{l}\text { AIR } \\
\text { ONLY }\end{array}$ & $\begin{array}{l}\text { AIR } \\
\text { ONLY }\end{array}$ & $\begin{array}{l}\text { AIR } \\
\text { CLAS }\end{array}$ & $\begin{array}{l}A I R \\
\text { MOL }\end{array}$ & $M U$ & $\begin{array}{l}3000 * \\
\text { LOG }\end{array}$ \\
\hline PRCT & $\begin{array}{l}\text { DEG } \\
\text { CENT }\end{array}$ & CPS & METRS & $\begin{array}{l}\text { WALL } \\
D R / S \\
3\end{array}$ & $\begin{array}{l}\text { WALL } \\
D B / S\end{array}$ & $\begin{array}{l}D B / S \\
4\end{array}$ & $/ M$ & $5^{/ M}$ & $/ M$ & 6 & $\mathrm{RH}$ \\
\hline
\end{tabular}

12345678901234567890123456789012345678901234567890123456789012345678

$\begin{array}{rrrrrrrrrrrrr}1.4 & 20 & 918 & .374 & 200 & 15.20 & 8.02 & 7.18 & 0482 & 1236 & 0470 & 0176 & 438 \\ 1.5 & 20 & 918 & .374 & 200 & 15.80 & 8.02 & 7.78 & 0522 & 1236 & 0510 & 0191 & 528 \\ 1.7 & 20 & 918 & .374 & 200 & 15.70 & 8.02 & 7.68 & 0516 & 1236 & 0504 & 0188 & 691 \\ 1.8 & 20 & 918 & .374 & 200 & 16.00 & 8.02 & 7.98 & 0536 & 1236 & 0524 & 0196 & 766 \\ 1.9 & 20 & 918 & .374 & 200 & 15.50 & 8.02 & 7.48 & 0502 & 1236 & 0490 & 0183 & 836 \\ 2.1 & 20 & 918 & .374 & 200 & 14.70 & 8.02 & 6.58 & 0448 & 1236 & 0436 & 0163 & 967 \\ 2.4 & 20 & 918 & .374 & 200 & 14.40 & 8.02 & 6.38 & 0428 & 1236 & 0416 & 0155 & 1141 \\ 3.1 & 20 & 918 & .374 & 200 & 13.40 & 8.02 & 5.38 & 0361 & 1236 & 0349 & 0130 & 1474 \\ 4.7 & 20 & 918 & .374 & 200 & 12.60 & 8.02 & 4.58 & 0307 & 1236 & 0295 & 0110 & 2016 \\ 6.4 & 20 & 918 & .374 & 200 & 11.00 & 8.02 & 2.98 & 0200 & 1236 & 0188 & 0070 & 2419 \\ 8.3 & 20 & 918 & .374 & 200 & 10.00 & 8.02 & 1.98 & 0133 & 1236 & 0121 & 0045 & 2757 \\ 16.7 & 20 & 918 & .374 & 200 & 9.20 & 8.02 & 1.18 & 0079 & 1236 & 0067 & 0025 & 3668 \\ 22.0 & 20 & 918 & .374 & 200 & 9.04 & 8.02 & 1.02 & 0068 & 1236 & 0056 & 0021 & 4027 \\ 26.3 & 20 & 918 & .374 & 200 & 9.00 & 8.02 & .98 & 0066 & 1236 & 0054 & 0020 & 4260\end{array}$

* last column employed in computer plotting only. 
TABLE NO. 7

TABULATION OF ORIGINAL DATA POINTS FOR

ABSORPTION OF SOUND IN DRY NITROGEN

AT 20, DEGREES CENTIGRADE

SPHERE CONTAINS TWO MICROPHONES++++NOMINAL VALUES OF FREQUENCY ARE LISTED

FREQUENCY 139 CPS, 298 CPS 512 CPS 724 CPS 933 CPS

PRESSURE RIN DB/SR IN DB/S R IN DB/SR IN DB/S R/IN DB/S

$\begin{array}{llllll}765 \mathrm{MM} & 2.14 & 1.39 & 1.20 & 2.06 & 4.31 \\ 493 \mathrm{MM} & 2.31 & 1.61 & 1.50 & 2.66 & 6.23 \\ 399 \mathrm{MM} & 2.42 & 1.75 & 1.68 & 2.92 & 6.98 \\ 298 \mathrm{MM} & 2.68 & 1.99 & 1.94 & 3.36 & 7.60 \\ 193 \mathrm{MM} & 3.08 & 2.41 & 2.44 & 4.20 & 8.05 \\ 148 \mathrm{MM} & 3.55 & 2.77 & 2.82 & 4.78 & 7.98\end{array}$


TABLE NO. 8

TABULATION OF SMOOTHED DATA POINTS FOR

ABSORPTION OF SOUND IN AIR VERSUS RELATIVE HUMIDITY

AT 20 DEGREES CENTIGRADE

\begin{tabular}{|c|c|c|c|c|c|c|c|c|c|c|c|}
\hline $\mathrm{R} \bullet \mathrm{H} \bullet$ & TEMP & FREQ & LAMDA & $\begin{array}{c}\text { AIR } \\
+\end{array}$ & & $\begin{array}{l}\text { AIR } \\
\text { ONLY }\end{array}$ & $\begin{array}{l}\text { AIR } \\
\text { ONLY }\end{array}$ & $\begin{array}{l}\text { AIR } \\
\text { CLAS }\end{array}$ & $\begin{array}{l}\text { AIR } \\
M O L\end{array}$ & MU & $\begin{array}{l}3000 * \\
\text { LOG }\end{array}$ \\
\hline PRCT & $\begin{array}{l}\text { DEG } \\
\text { CENT } \\
1\end{array}$ & CPS & $\begin{array}{l}\text { METRS } \\
2\end{array}$ & $\begin{array}{l}\text { WALL } \\
D B / S \\
3\end{array}$ & $\begin{array}{l}\text { WALL } \\
D B / S\end{array}$ & $\begin{array}{l}D B / S \\
4\end{array}$ & $/ M$ & $5^{/ M}$ & $/ M$ & 6 & $\mathrm{RH}$ \\
\hline
\end{tabular}

12345678901234567890123456789012345678901234567890123456789012345678

$\begin{array}{rlll}.1 & 20 & 136 & 2.520 \\ 1.2 & 20 & 136 & 2.520 \\ 1.4 & 20 & 136 & 2.520 \\ 1.8 & 20 & 136 & 2.520 \\ 2.1 & 20 & 136 & 2.520 \\ 2.7 & 20 & 136 & 2.520 \\ 3.8 & 20 & 136 & 2.520 \\ 5.1 & 20 & 136 & 2.520 \\ 6.1 & 20 & 136 & 2.520 \\ 6.2 & 20 & 136 & 2.520 \\ 7.2 & 20 & 136 & 2.520 \\ 7.4 & 20 & 136 & 2.520 \\ 8.2 & 20 & 136 & 2.520 \\ 9.0 & 20 & 136 & 2.520 \\ 10.1 & 20 & 136 & 2.520 \\ 13.3 & 20 & 136 & 2.520 \\ 16.3 & 20 & 136 & 2.520 \\ 20.0 & 20 & 136 & 2.520 \\ 25.4 & 20 & 136 & 2.520 \\ 31.0 & 20 & 136 & 2.520 \\ 39.0 & 20 & 136 & 2.520 \\ & & & \\ 1.2 & 20 & 293 & 1.170 \\ 1.4 & 20 & 293 & 1.170 \\ 1.8 & 20 & 293 & 1.170 \\ 2.1 & 20 & 293 & 1.170 \\ 2.7 & 20 & 293 & 1.170 \\ 3.8 & 20 & 293 & 1.170 \\ 5.1 & 20 & 293 & 1.170 \\ 6.1 & 20 & 293 & 1.170 \\ 6.2 & 20 & 293 & 1.170 \\ 7.2 & 20 & 293 & 1.170 \\ 7.4 & 20 & 293 & 1.170 \\ 8.2 & 20 & 293 & 1.170 \\ 9.0 & 20 & 293 & 1.170 \\ 10.1 & 20 & 293 & 1.170 \\ 13.3 & 20 & 293 & 1.170 \\ 16.3 & 20 & 293 & 1.170 \\ 20.0 & 20 & 293 & 1.170 \\ 25.4 & 20 & 293 & 1.170 \\ 31.0 & 20 & 293 & 1.170 \\ 39.0 & 20 & 293 & 1.170 \\ & & & \\ 103 & & \end{array}$

\begin{tabular}{|c|c|c|c|c|c|c|c|}
\hline 4 & 99 & 55 & 009 & 007 & 209 & & 00 \\
\hline 08 & & $\begin{array}{l}1.19 \\
1.09\end{array}$ & $\begin{array}{l}0085 \\
0083\end{array}$ & $\begin{array}{l}0007 \\
0007\end{array}$ & $\begin{array}{l}0085 \\
0083\end{array}$ & $\begin{array}{l}0202 \\
0184\end{array}$ & $\begin{array}{l}238 \\
438\end{array}$ \\
\hline & & .77 & 0074 & 0007 & 074 & & \\
\hline & & $\begin{array}{l}65 \\
51\end{array}$ & $\begin{array}{l}0065 \\
0048\end{array}$ & $\begin{array}{l}0007 \\
0007\end{array}$ & $\begin{array}{l}0065 \\
0048\end{array}$ & 0086 & $\begin{array}{r}96 \\
129\end{array}$ \\
\hline & & & 0032 & 0007 & 1032 & & 173 \\
\hline & & & $\begin{array}{l}23 \\
20\end{array}$ & $\begin{array}{l}0007 \\
0007\end{array}$ & $\begin{array}{l}23 \\
20\end{array}$ & & \\
\hline & & .37 & 0019 & 0007 & 0019 & 0063 & 2377 \\
\hline & & $\begin{array}{r}.33 \\
.27\end{array}$ & $\begin{array}{l}0017 \\
0017\end{array}$ & & & & $\begin{array}{l}2572 \\
2608\end{array}$ \\
\hline & & .36 & .7 & 17 & & 1 & 2741 \\
\hline & & & & & & & \\
\hline & & & & & 7 & & 301 \\
\hline & & .39 & 0017 & 0007 & 0017 & 0 & 3372 \\
\hline & & . 36 & & & & & 36 \\
\hline & & & & & & & \\
\hline & & .24 & 0017 & 0007 & 17 & & 4215 \\
\hline & & .23 & & $O C$ & & & 447 \\
\hline & & & & & & & 47 \\
\hline & & & & & & & \\
\hline & & & & & & & 43 \\
\hline & & & & & & & \\
\hline & & 1.82 & & 0032 & 0137 & & 12 \\
\hline & 36 & 1.50 & 0092 & 0 & & & 17 \\
\hline & & 1.07 & 0 & & & & \\
\hline & & .8 & 0054 & 00 & $O C$ & & 23 \\
\hline & & .86 & 0052 & 0032 & 0052 & 0 & 2377 \\
\hline & & 7 & 0045 & & & & 25 \\
\hline & & & 3 & 08 & 3 & & 2608 \\
\hline & & .71 & 0039 & 0032 & 0039 & 0 & 2741 \\
\hline & & & 0037 & & & & 286 \\
\hline & & & 0037 & & & & 3013 \\
\hline & & .6 & 0037 & 0032 & 0037 & & 3372 \\
\hline & & 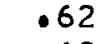 & 0037 & 0032 & 0037 & 00 & 3637 \\
\hline & & & 0037 & 0032 & 0037 & 00 & 3903 \\
\hline & & & & & 0037 & & 4215 \\
\hline & & & & $\begin{array}{l}0032 \\
0032\end{array}$ & & & 4474 \\
\hline & & & & & & & \\
\hline
\end{tabular}

* last column employed in computer plotting only. 
TABLE NO. 8

TABULATION OF SMOOTHED DATA POINTS FOR

ABSORPTION OF SOUND IN AIR VERSUS RELATIVE HUMIDITY AT 20 DEGREES CENTIGRADE

R.H. TEMP FREQ LAMDA DEG

PRCT CENT CPS METRS 1
A I R

$+$

WALL WALL $D B / S$ DB/S DB/S /M /M /M $34 \quad 5 \quad 5 \quad 6$
$3000 \%$

LOG

$\mathrm{RH}$

12345678901234567890123456789012345678901234567890123456789012345678

$\begin{array}{rrrr}.1 & 20 & 504 & .681 \\ 1.2 & 20 & 504 & .681 \\ 1.4 & 20 & 504 & .681 \\ 1.8 & 20 & 504 & .681 \\ 2.1 & 20 & 504 & .681 \\ 2.7 & 20 & 504 & .681 \\ 3.8 & 20 & 504 & .681 \\ 5.1 & 20 & 504 & .681 \\ 6.1 & 20 & 504 & .681 \\ 6.2 & 20 & 504 & .681 \\ 7.2 & 20 & 504 & .681 \\ 7.4 & 20 & 504 & .681 \\ 8.2 & 20 & 504 & .681 \\ 9.0 & 20 & 504 & .681 \\ 10.1 & 20 & 504 & .681 \\ 13.3 & 20 & 504 & .681 \\ 16.3 & 20 & 504 & .681 \\ 20.0 & 20 & 504 & .681 \\ 25.4 & 20 & 504 & .681 \\ 31.0 & 20 & .504 & .681 \\ 39.0 & 20 & 504 & .681 \\ & & & \\ 1.1 & 20 & 712 & .482 \\ 1.2 & 20 & 712 & .482 \\ 1.4 & 20 & 712 & .482 \\ 1.8 & 20 & 712 & .482 \\ 2.1 & 20 & 712 & .482 \\ 2.7 & 20 & 712 & .482 \\ 3.8 & 20 & 712 & .482 \\ 5.1 & 20 & 712 & .482 \\ 6.1 & 20 & 712 & .482 \\ 6.2 & 20 & 712 & .482 \\ 7.2 & 20 & 712 & .482 \\ 7.4 & 20 & 712 & .482 \\ 8.2 & 20 & 712 & .482 \\ 9.0 & 20 & 712 & .482 \\ 10.1 & 20 & 712 & .482 \\ 13.3 & 20 & 712 & .482 \\ 16.3 & 20 & 712 & .482 \\ 20.0 & 20 & 712 & .482 \\ 31.0 & 20 & 712 & .482 \\ 39.0 & 20 & 712 & .482\end{array}$

$1.81 \quad 1.17 \quad .64002000940020 \quad 00290000$

$4.761 .17 \quad 3.590232009402320163 \quad 238$

$5.301 .174 .130274009402740188 \quad 438$

$5.971 .174 .800320009403200219 \quad 766$

$6.121 .174 .95 \quad 032300940323 \quad 0225 \quad 967$

$5.56 \quad 1.17 \quad 4.390304009403040200 \quad 1294$

$\begin{array}{lllllllll}4.95 & 1.17 & 3.78 & 0229 & 0094 & 0229 & 0172 & 1739\end{array}$

$\begin{array}{lllllllll}3.92 & 1.17 & 2.75 & 0160 & 0094 & 0160 & 0125 & 2123\end{array}$

$\begin{array}{lllllllll}3.29 & 1.17 & 2.12 & 0131 & 0094 & 0131 & 0096 & 2356\end{array}$

$\begin{array}{lllllllll}3.26 & 1.17 & 2.09 & 0127 & 0094 & 0127 & 0095 & 2377\end{array}$

$2.931 .17 \quad 1.760108 .009401080080 \quad 2572$

$2.871 .17 \quad 1.70 \quad 0104009401040077 \quad 2608$

$2.761 .17 \quad 1.590091009400910072 \quad 2741$

$2.571 .17 \quad 1.40 \quad 0085009400850063 \quad 2853$

$2.521 .17 \quad 1.3500750094007500613013$

$2.291 .17 \quad 1.120055009400650050 \quad 3372$

$2.191 .17 \quad 1.02 \quad 0055 \quad 00940065 \quad 0046 \quad 3637$

$2.141 .17 \quad .9700550094006500443903$

2.121 .17 .9500650094006500434215

$2.151 .17 \quad .990055009400650044 \quad 4474$

$2.191 .17 \quad 1.02 \quad 0065 \quad 0094 \quad 00650046 \quad 4773$

$2.772 .07 \quad .70 \quad 00240187002300220000$

$\begin{array}{lllllllll}6.22 & 2.07 & 4.15 & 0259 & 0187 & 0258 & 0134 & 238\end{array}$

$\begin{array}{llllllllll}6.97 & 2.07 & 4.90 & 0301 & 0187 & 0300 & 0158 & 438\end{array}$

$8.30 \quad 2.07 \quad 6.23 \quad 03790187 \quad 0378 \quad 0201 \quad 766$

$\begin{array}{lllllllll}9.13 & 2.07 & 7.06 & 0429 & 0187 & 0428 & 0228 & 967\end{array}$

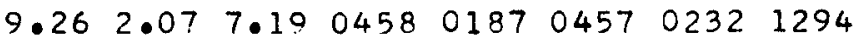

$\begin{array}{llllllllll}8.73 & 2.07 & 6.66 & 0411 & 0187 & 0410 & 0215 & 1739\end{array}$

$7.302 .075 .23 \quad 0315018703140168 \quad 2123$

$\begin{array}{lllllllll}6.21 & 2.07 & 4.14 & 0250 & 0187 & 0249 & 0133 & 2356\end{array}$

$\begin{array}{lllllllllll}6.19 & 2.07 & 4.12 & 0245 & 0187 & 0244 & 0133 & 2377\end{array}$

$\begin{array}{llllllllll}5.54 & 2.07 & 3.47 & 0204 & 0187 & 0203 & 0111 & 2572\end{array}$

$\begin{array}{lllllllllll}5.41 & 2.07 & 3.34 & 0199 & 0187 & 0198 & 0107 & 2608\end{array}$

$5.132 .073 .06 \quad 0176 \quad 018701750098 \quad 2741$

$\begin{array}{llllllllll}4.79 & 2.07 & 2.72 & 0158 & 0187 & 0157 & 0087 & 2863\end{array}$

$\begin{array}{llllllllll}4.74 & 2.07 & 2.67 & 0139 & 0187 & 0138 & 0085 & 3013\end{array}$

4.152 .072 .0801020187010100673372

$\begin{array}{lllllllll}3.89 & 2.07 & 1.82 & 0093 & 0187 & 0092 & 0058 & 3637\end{array}$

$\begin{array}{lllllllll}3.72 & 2.07 & 1.65 & 0093 & 0187 & 0092 & 0053 & 3903\end{array}$

$3.62 \quad 2.07 \quad 1.550093 \quad 0187 \quad 00920049 \quad 4215$

$\begin{array}{lllllllll}3.61 & 2.07 & 1.54 & 0093 & 0187 & 0092 & 0049 & 4474\end{array}$

3.652 .071 .5800930187009200504773

* last column employed in computer plotting only. 
TABLE NO. 8

TABULATION OF SMOOTHED DATA POINTS FOR

ABSORPTION OF SOUND IN AIR VERSUS RELATIVE HUMIDITY

AT 20 DEGREES CENTIGRADE

R.H. TEMP FREQ LAMDA

DEG

PRCT CENT CPS METRS 12

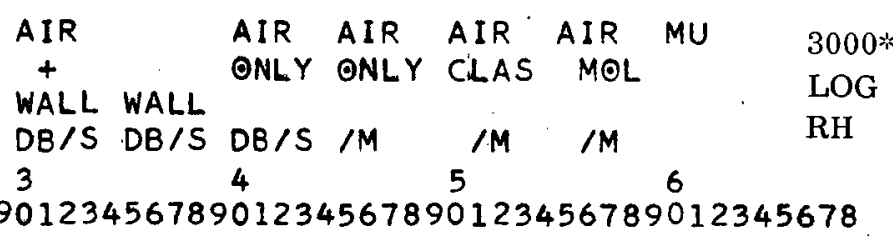

12345678901234567890123456789012345678901234567890123456789012345678

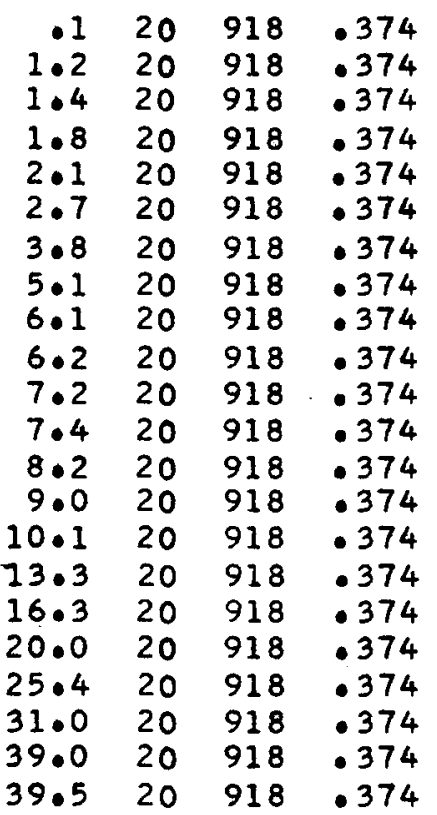

$\begin{array}{rrrllllr}5.22 & 4.50 & .72 & 0027 & 0312 & 0024 & 0017 & 0000 \\ 8.55 & 4.50 & 4.05 & 0291 & 0312 & 0288 & 0101 & 238 \\ 9.26 & 4.50 & 4.76 & 0333 & 0312 & 0330 & 0119 & 438 \\ 11.00 & 4.50 & 6.50 & 0417 & 0312 & 0414 & 0162 & 766 \\ 12.40 & 4.50 & 7.90 & 0489 & 0312 & 0486 & 0197 & 967 \\ 13.35 & 4.50 & 8.85 & 0585 & 0312 & 0582 & 0221 & 1294 \\ 13.20 & 4.50 & 8.70 & 0591 & 0312 & 0588 & 0217 & 1739 \\ 11.70 & 4.50 & 7.20 & 0483 & 0312 & 0480 & 0179 & 2123 \\ 10.35 & 4.50 & 5.85 & 0405 & 0312 & 0402 & 0146 & 2356 \\ 10.30 & 4.50 & 5.80 & 0389 & 0312 & 0386 & 0144 & 2377 \\ 9.48 & 4.50 & 4.98 & 0327 & 0312 & 0324 & 0124 & 2572 \\ 9.26 & 4.50 & 4.76 & 0315 & 0312 & 0312 & 0119 & 2608 \\ 8.85 & 4.50 & 4.35 & 0285 & 0312 & 0282 & 0108 & 2741 \\ 8.33 & 4.50 & 3.83 & 0255 & 0312 & 0252 & 0095 & 2863 \\ 8.25 & 4.50 & 3.75 & 0225 & 0312 & 0222 & 0093 & 3013 \\ 7.25 & 4.50 & 2.75 & 0165 & 0312 & 0162 & 0068 & 3372 \\ 6.90 & 4.50 & 2.40 & 0135 & 0312 & 0132 & 0059 & 3637 \\ 6.50 & 4.50 & 2.00 & 0123 & 0312 & 0120 & 0049 & 3903 \\ 6.28 & 4.50 & 1.78 & 0123 & 0312 & 0120 & 0043 & 4215 \\ 6.23 & 4.50 & 1.73 & 0123 & 0312 & 0120 & 0042 & 4474 \\ 6.28 & 4.50 & 1.78 & 0123 & 0312 & 0120 & 0043 & 4773 \\ 6.03 & 4.50 & 1.53 & 0123 & 0312 & 0120 & 0037 & 4790\end{array}$

* last column employed in computer plotting only. 
TABLE NO. 9

TABULATION OF SMOOTHED DATA POINTS FOR

ABSORPTION OF SOUND IN AIR VERSUS RELATIVE HUMIDITY AT O DEGREES CENTIGRADE

\begin{tabular}{|c|c|c|c|c|c|c|c|c|c|c|c|}
\hline $\mathrm{R} \cdot \mathrm{H}=$ & TEMP & FREQ & LAMOA & $\begin{array}{c}\text { AIR } \\
+\end{array}$ & $\therefore$ & $\begin{array}{l}\text { AIR } \\
\text { ONLY }\end{array}$ & $\begin{array}{l}\text { AIR } \\
\text { ONLY }\end{array}$ & $\begin{array}{l}\text { AIR } \\
\text { CLAS }\end{array}$ & $\begin{array}{l}A I R \\
M \odot L\end{array}$ & $M U$ & $\begin{array}{l}3000 \% \\
\text { LOG }\end{array}$ \\
\hline$P R C T$ & $\begin{array}{l}\text { DEG } \\
\text { CENT }\end{array}$ & CPS & $\begin{array}{c}\text { METRS } \\
2\end{array}$ & $\begin{array}{l}\text { WALL } \\
D B / S \\
3\end{array}$ & $\begin{array}{l}\text { WALL } \\
D B / S\end{array}$ & $\begin{array}{l}D B / S \\
4\end{array}$ & $/ M$ & $5^{/ M}$ & $/ M$ & 6 & $\mathrm{RH}$ \\
\hline
\end{tabular}

12345678901234567890123456789012345678901234567890123456789012345678

\begin{tabular}{|c|c|c|c|c|c|c|c|c|c|c|c|}
\hline $3 \cdot 1$ & 0 & 132 & 2.520 & 2.60 & 1.88 & .72 & 0053 & 0007 & 0053 & 0126 & 1474 \\
\hline $4 \cdot 1$ & 0 & 132 & 2.520 & 2.67 & 1.88 & .79 & 0060 & 0007 & 0060 & 0138 & 1838 \\
\hline $4 \cdot 4$ & 0 & 132 & 2.520 & 2.67 & 1.88 & .79 & 0050 & 0007 & 0060 & 0138 & 1930 \\
\hline $5 \cdot 4$ & 0 & 132 & 2.520 & 2.64 & 1.88 & .76 & 0058 & 0007 & 0058 & 0133 & 2197 \\
\hline $6 \cdot 2$ & 0 & 132 & 2.520 & 2.53 & 1.88 & .65 & 0053 & 0007 & 0053 & 0113 & 2377 \\
\hline $7 \cdot 3$ & 0 & 132 & 2 & & 8 & .67 & 0046 & 0007 & 0046 & 0118 & 2590 \\
\hline $8 \cdot 9$ & 0 & 132 & 2.520 & 2.34 & 1.88 & .46 & 0037 & 0007 & 0037 & 0080 & 2848 \\
\hline $11 \cdot 1$ & 0 & 132 & 2.520 & 2.37 & 1.88 & .49 & 0028 & 0007 & 0028 & 0085 & 3136 \\
\hline 1.9 & 0 & 132 & 520 & 2.26 & 1.88 & .38 & 0026 & 0007 & 0026 & 0065 & 3227 \\
\hline 12 & 0 & 132 & 2 & 2 & 8 & .29 & 0 & 0007 & 5 & 150 & 3280 \\
\hline 13.0 & 0 & 132 & 2.520 & 2.10 & 1.88 & .22 & 0023 & 0007 & 0023 & 0038 & 3342 \\
\hline 15.4 & 0 & 132 & 2.520 & 2.10 & 1.88 & .22 & 0019 & 0007 & 0019 & 0038 & 3563 \\
\hline 17.5 & 0 & 132 & 2.520 & 2.07 & 1.88 & .19 & 0017 & 0007 & 0017 & 0033 & 3729 \\
\hline $25 \cdot 1$ & 0 & 132 & 20 & 2.04 & 8 & .16 & 0012 & 0007 & 0012 & 0028 & 4199 \\
\hline 27.4 & 0 & 132 & 2.520 & $? .0 ?$ & 1.88 & .14 & 0012 & 0007 & 0012 & 0 & 4313 \\
\hline 33.6 & 0 & 132 & 2.520 & 2.02 & 1.88 & .14 & 0012 & 0007 & 0012 & 0025 & 4579 \\
\hline 38.4 & 0 & 132 & 2.520 & 1.97 & 1.88 & .09 & 0012 & 0007 & 0012 & 0015 & 4753 \\
\hline $44 \cdot 6$ & 0 & 132 & 20 & 2.02 & 1.88 & .14 & 0012 & 0007 & 0012 & 0025 & 4948 \\
\hline $54 \cdot 0$ & 0 & 132 & 2.520 & 1.99 & 1.88 & .11 & 0012 & 0007 & 0012 & 0020 & 5197 \\
\hline 64.0 & 0 & 132 & 20 & 3 & 1.88 & .15 & 0012 & 0007 & 0012 & 0025 & 5419 \\
\hline 84.0 & 0 & 132 & 20 & 1.99 & 1.88 & .11 & 0012 & 0007 & 0012 & 0020 & 5773 \\
\hline 91.0 & 0 & 132 & 20 & 2.00 & 1.88 & .12 & 0012 & 0007 & 0012 & 0020 & 5877 \\
\hline - 1 & 0 & $28 ?$ & 1.170 & 1.63 & ]. .34 & .29 & 0001 & 0031 & 0001 & 0024 & 0000 \\
\hline $3 \cdot 1$ & 0 & 282 & 1.170 & 2.49 & 1.34 & 1.15 & 0070 & 0031 & 0070 & 0094 & 1474 \\
\hline $\begin{array}{l}4 \cdot 1 \\
4.4\end{array}$ & $\begin{array}{l}0 \\
0\end{array}$ & $\begin{array}{l}282 \\
282\end{array}$ & $\begin{array}{l}1.170 \\
1.170\end{array}$ & & $\begin{array}{l}1.34 \\
1.34\end{array}$ & $\begin{array}{l}1.41 \\
1.58\end{array}$ & $\begin{array}{l}0089 \\
0096\end{array}$ & $\begin{array}{l}0031 \\
0031\end{array}$ & $\begin{array}{l}0089 \\
0096\end{array}$ & $\begin{array}{l}01.15 \\
0129\end{array}$ & $\begin{array}{l}1838 \\
1930\end{array}$ \\
\hline 5.4 & 0 & 282 & 1.170 & 3.05 & $1 \cdot 34$ & 1.71 & 0106 & 0031 & 0106 & 0140 & 2197 \\
\hline 6.2 & 0 & 282 & 1. & 3.08 & 1.34 & $1 \cdot 74$ & 0123 & 0031 & 0123 & 0142 & 2377 \\
\hline 7. & 0 & 282 & 1. & .14 & 1.34 & 1.80 & 0123 & 0031 & 0123 & 0147 & 2590 \\
\hline 8.9 & 0 & 282 & 1.170 & 3.07 & 1.34 & 1.73 & 0119 & 0031 & 0119 & 0141 & 2848 \\
\hline 11.1 & 0 & 282 & 1.170 & 2.95 & 1.34 & 1.61 & 0101 & 0031 & 0101 & 0132 & 3136 \\
\hline 11.9 & 0 & 282 & 1. & & $1 \cdot 34$ & 1.41 & 0095 & 0031 & 0095 & 0115 & 3227 \\
\hline 12.4 & 0 & 282 & 1.170 & 2.58 & 1.34 & 1.24 & 0091 & 0031 & 0091 & 0101 & 3280 \\
\hline 13.0 & 0 & 282 & 1.170 & .39 & 1.34 & 1.05 & 0087 & 0031 & 0087 & 0086 & 3342 \\
\hline 15.4 & 0 & 282 & .70 & 22 & $1 \cdot 34$ & .88 & 0070 & 0031 & 0070 & 0072 & 3563 \\
\hline - 5 & 0 & 282 & 0 & 6 & 1.34 & .72 & 0060 & 0031 & 0060 & 0059 & 372 \\
\hline $25 \cdot 1$ & 0 & 282 & 1.170 & 8 & $1 \cdot 34$ & . 54 & 0040 & 0031 & 0040 & 0045 & 4199 \\
\hline 27.4 & 0 & 282 & 1.170 & 37 & 1.34 & .53 & 0036 & 0031 & 0036 & 0043 & 4313 \\
\hline 33.6 & 0 & 282 & .170 & 4 & 1.34 & .40 & 0029 & 0031 & 0029 & 0033 & 4579 \\
\hline 38.4 & 0 & 282 & .170 & 8 & .34 & .35 & 0026 & 0031 & 0026 & 0028 & 4753 \\
\hline 4 & 0 & 282 & 1.170 & 5 & 1.34 & .31 & 0025 & 0031 & 0025 & 0026 & 4948 \\
\hline 54.0 & 0 & 282 & 1.170 & 34 & $1 \cdot 34$ & .30 & 0025 & 0031 & 0025 & 0025 & 5197 \\
\hline 64.0 & 0 & 282 & .170 & & $1 \cdot 34$ & $\bullet<5$ & 0025 & 0031 & 0025 & 0020 & 5419 \\
\hline 84.0 & 0 & 282 & 1.170 & & $1 \cdot 34$ & .2 & 0025 & 0031 & 0025 & 0020 & 5773 \\
\hline 91.0 & 0 & 2.82 & 1.170 & 1.59 & 1.34 & & 0025 & 0031 & 0025 & 0020 & 5877 \\
\hline
\end{tabular}


TABLE NO. 9

TABULATION OF SMOOTHED DATA POINTS FOR

ABSORPTION OF SOUND IN AIR VERSUS RELATIVE HUMIDITY

AT O DEGREES CENTIGRADE

$\mathrm{R} \bullet H \bullet T E M P$ FREQ LAMDA

DEG

PRCT CENT CPS METRS 112

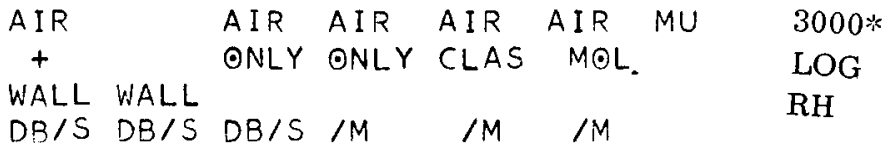
$3 \quad 4 \quad 5 \quad 6$

12345678901234567890123456789012345678901234567890123456789012345678

\begin{tabular}{|c|c|c|c|c|c|c|c|c|c|c|c|}
\hline - 1 & 0 & 486 & .681 & 1.43 & $1 \cdot 10$ & .33 & 0002 & 0090 & 0006 & & \\
\hline $3 \cdot 1$ & 0 & 486 & .681 & 2.40 & 1.10 & $1 \cdot 30$ & 0083 & 0090 & 0083 & 0061 & 1474 \\
\hline $4 \cdot 1$ & 0 & 486 & 681 & 2.76 & 1.10 & 1.66 & 0109 & 0090 & 0109 & 78 & 838 \\
\hline 4.4 & 0 & 486 & .681 & 2.98 & 1.10 & 1.88 & 0117 & 0090 & 117 & 0089 & 33 \\
\hline 5.4 & 0 & 486 & 681 & 3.32 & 1.10 & 2.22 & 0143 & 0090 & 143 & 0104 & \\
\hline 6.2. & 0 & 486 & .681 & 3.54 & 1.10 & 2.44 & 0160 & 0090 & 160 & 0115 & 3 \\
\hline $7 \cdot 3$ & 0 & 486 & .68 & 3.78 & 1.10 & 2.68 & 0192 & 0090 & 2 & 0126 & \\
\hline 8.9 & 0 & 486 & .681 & 4.03 & 1.10 & $2 \cdot 93$ & 0209 & 0090 & 0209 & 0138 & 284 \\
\hline $1 \cdot 1$ & 0 & 486 & .6 & 4.13 & 1.10 & 3.03 & 0209 & 0090 & 0209 & 0143 & 12 \\
\hline 1.9 & 0 & 486 & .681 & 4.12 & 1.10 & 3.02 & 0207 & 0090 & 0207 & 0143 & 322 \\
\hline $2 \cdot 4$ & 0 & 486 & . 681 & 3.97 & 1.10 & 2.87 & 0204 & 0090 & 0204 & 0136 & $\therefore$ \\
\hline 3.0 & $0^{\circ}$ & 486 & .6 & 3.71 & 1.10 & 2.61 & 0200 & 0 & 00 & 0123 & 334 \\
\hline $5 \cdot 4$ & 0 & 486 & .681 & 3.37 & 1.10 & $2 \cdot 27$ & 0177 & 0090 & 7 & 0107 & 356 \\
\hline $7 \cdot 5$ & 0 & 486 & .6 & 3.05 & 1.10 & 1.95 & 0158 & 0090 & 58 & 0092 & 372 \\
\hline $5 \cdot 1$ & 0 & 486 & .681 & 2.58 & 1.10 & 1.48 & 0102 & 0090 & 2 & 0070 & 419 \\
\hline $7 \cdot 4$ & 0 & 486 & .6 & 2.57 & $1 \cdot 10$ & 1.47 & 0092 & 0090 & 2 & 0069 & 431 \\
\hline 33.6 & 0 & 486 & .681 & 2.16 & 1.10 & 1.06 & 0072 & 0090 & 0072 & 0050 & 457 \\
\hline $38 \cdot 4$ & 0 & 486 & .6 & 2.00 & 1.10 & .90 & 0062 & 0090 & 0062 & 0042 & 475 \\
\hline $44 \cdot 6$ & 0 & 486 & .681 & & 1.10 & .78 & 0053 & 0090 & 0053 & 0036 & 494 \\
\hline 54.0 & 0 & 486 & .681 & 1.81 & 1.10 & .71 & 0045 & 0090 & 0045 & 0033 & 519 \\
\hline 64.0 & 0 & 486 & .6 & 1.67 & 1.10 & .57 & 0042 & 0090 & 0042 & 0027 & 541 \\
\hline 84 & 0 & 486 & - 681 & 61 & $1 \cdot 10$ & .51 & 0042 & 0090 & 0042 & 0023 & 577 \\
\hline 1.0 & 0 & 486 & .68 & 59 & $1 \cdot 10$ & .49 & 0042 & 00 & & 0023 & 587 \\
\hline$\cdot 1$ & 0 & 686 & .4 & & $2 \cdot 62$ & .08 & 0004 & 19 & 03 & 0002 & 000 \\
\hline $3 \cdot 1$ & 0 & 686 & .482 & 3.77 & 2.62 & 1.15 & 0094 & 0179 & 0093 & 0038 & 147 \\
\hline $4 \cdot 1$ & 0 & 686 & .482 & 4.03 & 2.62 & 1.41 & 0125 & 0179 & 0124 & 0046 & 183 \\
\hline $4 \cdot 4$ & 0 & 686 & .432 & $4 \cdot 38$ & 2.62 & 1.76 & 0131 & 0179 & 0130 & 0058 & 193 \\
\hline $5 \cdot 4$ & 0 & 686 & .482 & 4.79 & 2.62 & 2.17 & 0158 & 0179 & 0157 & 0072 & 219 \\
\hline $6 \cdot 2$ & 0 & 686 & .482 & $5 \cdot 14$ & 2.62 & 2.52 & 0182 & 0179 & 0181 & 0084 & 237 \\
\hline $7 \cdot 3$ & 0 & 686 & .482 & 5.49 & 2.62 & 2.87 & 0209 & 0179 & 0208 & 0096 & 259 \\
\hline $8 \cdot 9$ & 0 & 685 & .482 & 5.97 & $2 \cdot 62$ & $3 \cdot 35$ & 0255 & 0179 & 0254 & 0112 & 284 \\
\hline $1 \cdot 1$ & 0 & 686 & .482 & 6.28 & 2.62 & 3.66 & 0294 & 0179 & 0293 & 0122 & 313 \\
\hline 1.9 & 0 & 686 & .482 & 6.58 & 2.62 & 3.96 & 0300 & 0179 & 0299 & 0132 & 322 \\
\hline $12 \cdot 4$ & 0 & 686 & .482 & 6.70 & 2.62 & 4.08 & 0303 & 0179 & 0302 & 0136 & 328 \\
\hline 13.0 & 0 & 686 & .482 & 6.63 & 2.52 & 4.01 & 0308 & 0179 & 0307 & 0134 & 334 \\
\hline 15.4 & 0 & 686 & .482 & 6.28 & 2.62 & 3.66 & 0295 & 0179 & 0296 & 0.122 & 356 \\
\hline 17.5 & 0 & 686 & .482 & 5.80 & $2 \cdot 62$ & 3.18 & 0275 & 0179 & 02.74 & 0106 & 372 \\
\hline 25.1 & 0 & 685 & .482 & 5.03 & 2.62 & 2.41 & 0200 & 0179 & 0199 & 0080 & 419 \\
\hline 27.4 & 0 & 686 & .482 & 5.16 & 2.62 & 2.54 & 0176 & 0179 & 0175 & 0085 & 431 \\
\hline 33.6 & 0 & 686 & .482 & 4.33 & 2.62 & 1.71 & 0140 & 0179 & 0139 & 0057 & 457 \\
\hline $8 \cdot 4$ & 0 & 686 & .482 & 4.03 & 2.62 & 1.41 & 0122 & 0179 & 0121 & 0046 & 475 \\
\hline $4 \cdot 6$ & 0 & 686 & .482 & & 2.62 & 1.15 & 0101 & 0179 & 0100 & 0038 & 494 \\
\hline 54.0 & 0 & 686 & .482 & 3.62 & 2.62 & 1.00 & 0083 & 0179 & 0082 & 0033 & 519 \\
\hline 64.0 & 0 & 686 & .482 & 3.37 & 2.62 & .75 & 0070 & 0179 & 0069 & 0024 & 541 \\
\hline 34.0 & 0 & 686 & .482 & 3.26 & 2.62 & .64 & 0061 & 0179 & 0060 & 0020 & 577 \\
\hline 1.0 & 0 & 686. & .482 & 3.13 & 2.62 & .51 & 0061 & 0179 & 0060 & 0016 & 587 \\
\hline
\end{tabular}

* last column employed in computer plotting only. 
TARLE NO. 9

TABULATIGN OF SMOQTHFD DATA POINTS FOR

ABSORPTION OF SOUND IN AIR VERSUS RELATIVE HUMIDITY

AT O DFGREFS CFNTIGPADE

\begin{tabular}{|c|c|c|c|c|c|c|c|c|c|c|c|}
\hline $\mathrm{R} . \mathrm{H}$. & TEMP & FREQ & $\angle \triangle M D A$ & $A I P$ & & AIR & $A I R$ & $A I R$ & $A I R$ & MU & $3000 *$ \\
\hline & DEG & & & $\stackrel{+}{\text { WALL }}$ & WALL & $\odot N L Y$ & ONLY & CLAS & IAOL & & $\begin{array}{l}\mathrm{LOG} \\
\mathrm{RH}\end{array}$ \\
\hline PRCT & $\begin{array}{l}\text { CENT } \\
1\end{array}$ & CPS & $\begin{array}{l}\text { METPS } \\
2\end{array}$ & $\begin{array}{l}D E / S \\
3\end{array}$ & $D E / S$ & $D B / S$ & $/ M$ & $-1 M$ & $\operatorname{lin}$ & & \\
\hline
\end{tabular}

12345678901234567890123456789012345678901234567890123456789012345678

\begin{tabular}{|c|c|c|c|c|c|c|c|c|c|c|c|}
\hline .1 & 0 & 886 & .374 & 4.57 & 4.00 & .57 & 0006 & 0299 & 0004 & 0014 & 0000 \\
\hline $3 \cdot 1$ & 0 & 886 & .374 & 5.58 & 4.00 & 1.58 & 0115 & 0299 & 0113 & 0040 & 474 \\
\hline $4 \cdot 1$ & 0 & 886 & .374 & 6.08 & 4.00 & 2.08 & 01.46 & 0299 & 0144 & 0053 & 1838 \\
\hline 4.4 & 0 & 885 & 374 & 6.13 & 4.00 & 2.13 & 0158 & 0299 & 0156 & 0054 & 930 \\
\hline 5.4 & 0 & 886 & 374 & 6.65 & 4.00 & $2 \cdot 65$ & 0193 & 0.299 & 0191 & 068 & 197 \\
\hline 6.2 & 0 & 886 & .374 & 7.10 & 4.00 & 3.10 & $02 ? 1$ & 0299 & 0219 & 0090 & 377 \\
\hline $7 \cdot 3$ & 0 & 886 & .374 & & 4.00 & 3.43 & 0252 & 0299 & $0 \geq 50$ & 088 & 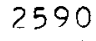 \\
\hline 8.9 & 0 & 886 & .374 & 8.29 & 4.00 & 4.29 & 0,306 & 0299 & 0304 & 110 & 848 \\
\hline $1 \cdot 1$ & 0 & 886 & .374 & 8.69 & 4.00 & 4.68 & 0372 & 0299 & 0370 & & 136 \\
\hline $1 \cdot 9$ & 0 & 886 & .374 & 9.40 & 4.00 & 5.40 & 0378 & 0299 & 0378 & 9 & 227 \\
\hline 2.4 & 0. & 886 & .374 & 9.78 & 4.00 & 5.78 & 03 & 0299 & 386 & & 280 \\
\hline 3.0 & 0 & 886 & - 374 & 10.00 & 4.00 & 6.00 & 0392 & 0299 & 0390 & & 34 \\
\hline $5 \cdot 4$ & 0 & 886 & .374 & & 4.00 & 5.67 & & 99 & 86 & 46 & 56 \\
\hline $7 \cdot 5$ & 0 & 886 & - 374 & & 4.00 & 5.45 & 03 & 02 & 90 & & 72 \\
\hline 27.4 & 0 & 886 & .374 & & 4.00 & 4.36 & 0256 & 0299 & $0 ? 54$ & 12 & 4313 \\
\hline 33.6 & 0 & 886 & .374 & 7.40 & 4.00 & 3.40 & 0197 & $029 \circ$ & 0195 & 87 & +57 \\
\hline 38.4 & 0 & 886 & .374 & 6.96 & 4.00 & 2.95 & 0170 & 0299 & 0168 & 0076 & 4753 \\
\hline $44 \cdot 6$ & 0 & 886 & .374 & & 4.00 & 2.52 & $01<3$ & 0299 & 0141 & 0064 & 4948 \\
\hline 54.0 & 0 & 886 & . 274 & 6.28 & 4.00 & 2.29 & 0115 & 0299 & 0113 & 058 & 5197 \\
\hline 4.0 & 0 & 886 & .374 & & 4.00 & 1.77 & 0009 & 0259 & 0007 & 0045 & 5419 \\
\hline $34 \cdot 0$ & 0 & 886 & . 374 & 5.42 & 4.00 & 1.42 & 0080 & 6299 & 078 & 36 & 578 \\
\hline 91.0 & 0 & 886 & 374 & 5.3 & 4.0 & $1 \cdot 36$ & 009 & 0299 & 0078 & 34 & 87 \\
\hline
\end{tabular}

* last column employed in computer plotting only. 
TARLF NO. 10

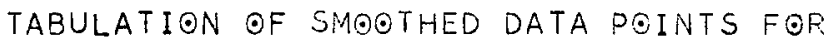

ABSORPTION OF SOUND IN AIR VERSUS RELATIVE HUMIDITY AT -20 DEGREES CENTIGRADE .

\begin{tabular}{|c|c|c|c|c|c|c|c|c|c|c|c|}
\hline . H. & TEMP & FREQ & LAMDA & $\begin{array}{c}\text { AIR } \\
+\end{array}$ & & $\begin{array}{l}\text { AIR } \\
\text { ONLY }\end{array}$ & $\begin{array}{l}\text { AIR } \\
\text { ONLY }\end{array}$ & $\begin{array}{l}\text { AIR } \\
\text { CLAS }\end{array}$ & $\begin{array}{l}\text { AIR } \\
\text { MOL }\end{array}$ & MU & $\begin{array}{l}3000 * \\
\text { LOG }\end{array}$ \\
\hline 0 & $\begin{array}{l}\text { DEG } \\
\text { CENT }\end{array}$ & CPS & $\begin{array}{l}\text { METRS } \\
2\end{array}$ & $\begin{array}{l}\text { WALL } \\
D B / S \\
3\end{array}$ & $\begin{array}{l}\text { WALL } \\
D B / S\end{array}$ & $\begin{array}{l}D B / S \\
4\end{array}$ & $/ M$ & $5^{/ M}$ & $/ M$ & 6 & $\mathrm{RH}$ \\
\hline
\end{tabular}

12345678901234567890123456789012345678901234567890123456789012345678

$\begin{array}{llll}17.7 & -20 & 272 & 1.170 \\ 22.2 & -20 & 272 & 1.170 \\ 29.3 & -20 & 272 & 1.170 \\ 31.1 & -20 & 272 & 1.170 \\ 37.0 & -20 & 272 & 1.170 \\ 42.8 & -20 & 272 & 1.170 \\ 46.7 & -20 & 272 & 1.170 \\ 58.0 & -20 & 272 & 1.170 \\ 66.5 & -20 & 272 & 1.170 \\ 72.0 & -20 & 272 & 1.170 \\ 78.0 & -20 & 272 & 1.170 \\ 80.0 & -20 & 272 & 1.170 \\ 98.0 & -20 & 272 & 1.170\end{array}$

$17.7-20 \quad 468 \quad .680$

$22.2-20 \quad 468 \quad .580$

$29.3-20 \quad 468 \quad .680$

$31 \cdot 1-20 \quad 468 \quad .680$

$37.0 \quad-20 \quad 468 \quad \cdot 680$

$42.8-20 \quad 468 \quad .680$

$\begin{array}{llll}46.7 & -20 & 468 & .680\end{array}$

$58.0 \quad-20 \quad 468 \quad .680$

$66.5 \quad-20 \quad 468 \quad .680$

$\begin{array}{llll}72.0 & -20 & 468 & .680\end{array}$

$\begin{array}{llll}78.0 & -20 & 468 & .680\end{array}$

$80.0 \quad-20 \quad 468 \quad .680$

$98 \cdot 0 \quad-20 \quad 468 \quad .680$
1.651 .21

1.901 .21

2.101 .21

2.161 .21

2.161 .21

2.121 .21

2.051 .21

1.951 .21

1.861 .21

1.791 .21

$1.73 \quad 1.21$

1.711 .21

$1.68 \mathrm{i} .21$

$\begin{array}{llllll}.44 & 0044 & 0029 & 0044 & 0038 & 3744 \\ .69 & 0058 & 0029 & 0058 & 0059 & 4039 \\ .89 & 0066 & 0029 & 0066 & 0075 & 4401 \\ .95 & 0067 & 0029 & 0067 & 0081 & 4478 \\ .95 & 0057 & 0029 & 0067 & 0081 & 4705 \\ .91 & 0062 & 0029 & 0062 & 0077 & 4894 \\ .84 & 0058 & 0029 & 0058 & 0072 & 5008 \\ .74 & 0049 & 0029 & 0049 & 0062 & 5290 \\ .65 & 0044 & 0029 & 0044 & 0055 & 5468 \\ .58 & 0042 & 0029 & 0042 & 0049 & 5572 \\ .52 & 0039 & 0029 & 0039 & 0045 & 5676 \\ .50 & 0038 & 0029 & 0038 & 0042 & 5709 \\ .47 & 0032 & 0029 & 0032 & 0040 & 5974\end{array}$

$\begin{array}{lllllllll}1.45 & .99 & .46 & 0037 & 0086.0036 & 0022 & 3744\end{array}$

$\begin{array}{llllllll}1.78 & .99 & .79 & 0056 & 0086 & 0055 & 0038 & 4039\end{array}$

2.04 .991 .0500900086008900514401

$\begin{array}{lllllllll}2.34 & .99 & 1.35 & 0098 & 0086 & 0097 & 0066 & 4478\end{array}$

$\begin{array}{llllllllll}2.55 & .99 & 1.56 & 0113 & 0086 & 0112 & 0076 & 4705\end{array}$

$2.63 \quad .991 .5401190086011800804894$

$\begin{array}{lllllllll}2.64 & .99 & 1.65 & 0121 & 0086 & 0120 & 0080 & 5008\end{array}$

$2.62 \quad .99 \quad 1.63 \quad 01180086 \quad 0117 \quad 0080 \quad 5290$

$2.53 \quad .991 .540110008601090075 \quad 5468$

$2.42 \quad .991 .43010300860102 \quad 00705572$

$2.31 \quad .991 .3200980086 \quad 009700645676$

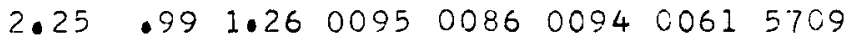

$2.10 \quad .991 .1100820086008100545974$

* last column employed in computer plotting only. 
TARLE NO. 10

TABULATION OF SMOOTHED DATA POINTS FOR .

ABSORPTION OF SQUND IN AIF VERSUS RELATIVE HUMIOITY AT -20 DEGREES CENTIGRADE

\begin{tabular}{|c|c|c|c|c|c|c|c|c|c|c|c|}
\hline $\mathrm{R} \bullet \mathrm{H} \bullet$ & TEMP & FREQ & LAMDA & $\begin{array}{c}\text { AIR } \\
+\end{array}$ & & $\begin{array}{l}\text { AIR } \\
\text { ONLY }\end{array}$ & $\begin{array}{l}\text { AIR } \\
\text { ONLY }\end{array}$ & $\begin{array}{l}\text { AIR } \\
\text { CLAS }\end{array}$ & $\begin{array}{l}A I P \\
\because \odot L\end{array}$ & $\forall U$ & $\begin{array}{l}3000 * \\
\text { LOG }\end{array}$ \\
\hline & $\begin{array}{l}\text { DEG } \\
\text { CENT }\end{array}$ & CPS & METRS & $\begin{array}{l}W A L L \\
D B / S\end{array}$ & $\begin{array}{l}\text { WALL } \\
D B / S\end{array}$ & $3 / 5$ & $\lim$ & $/ M$ & $/ M$ & & $\mathrm{RH}$ \\
\hline
\end{tabular}

12345678901234567890123456789012345678701234567890123456789012345678

\begin{tabular}{|c|c|c|c|c|c|c|c|c|c|c|c|}
\hline 17.7 & -20 & 660 & .482 & 2.32 & 1.86 & .46 & 0033 & 0170 & 0031 & 0015 & 3744 \\
\hline $22 . ?$ & -20 & 660 & .482 & 2.66 & 1.86 & .80 & 0052 & 0170 & 0050 & 0027 & 4039 \\
\hline $29 \cdot 3$ & -20 & 660 & .482 & 2.98 & 1.86 & 1.12 & 0096 & 0170 & 0094 & 0038 & 4401 \\
\hline $1 \cdot 1$ & -20 & 660 & .482 & 3.40 & 1.86 & 1.54 & 0110 & 0170 & 0108 & 0053 & 4478 \\
\hline 7.0 & -20 & 660 & .482 & 3.73 & 1.85 & 1.87 & 0139 & 0170 & 0137 & 0064 & 4705 \\
\hline $42 \cdot 8$ & -20 & 660 & .482 & 3.99 & 1.86 & 2.13 & 0159 & 0170 & 0157 & $\cot 3$ & 4894 \\
\hline 46.7 & -20 & 660 & .482 & 4.14 & 1.86 & 2.28 & 0166 & 0170 & 0164 & 0079 & 5008 \\
\hline 58.0 & -20 & 660 & .482 & 4.25 & 1.86 & 2.39 & 0174 & 0170 & 0172 & 0083 & 5290 \\
\hline 66.5 & -20 & 660 & .482 & 4.23 & 1.86 & 2.37 & 0172 & 0170 & 0170 & 0082 & 5468 \\
\hline 72.0 & -20 & 660 & .482 & $4 \cdot 20$ & 1.36 & 7.34 & 01.67 & 0170 & 0165 & 0081 & 5572 \\
\hline & -20 & 660 & .482 & 08 & 1.86 & 2.22 & 0159 & 0170 & 0157 & 0076 & 5576 \\
\hline 80.0 & -20 & 660 & .482 & 4.04 & 1.86 & 2.28 & 0157 & 0170 & 0155 & 0075 & 5709 \\
\hline 98 & -20 & 660 & .487 & 36 & 1.36 & 2.10 & 0135 & 0170 & 0133 & $\mathrm{C} 072$ & 5974 \\
\hline 17.7 & -20 & 853 & .373 & 3.82 & 3 & .24 & 0021 & 0288 & 001.8 & 0005 & 3744 \\
\hline 22 & -20 & 853 & $\begin{array}{r}37 \\
-37\end{array}$ & 5 & 8 & .47 & 0045 & 0288 & 0042 & $\mathrm{CO} 12$ & 4039 \\
\hline & -20 & 853 & .37 & 38 & & .80 & 0080 & 0288 & 0077 & 0021 & 4401 \\
\hline $31 \cdot 1$ & -20 & 853 & .373 & 4.83 & 3.58 & 1.25 & 0089 & 0288 & 0086 & 0033 & 4478 \\
\hline 37.0 & -20 & 853 & .373 & 5.27 & 3.58 & 1.69 & 0123 & 0288 & 0120 & 0045 & 4705 \\
\hline 42.8 & -20 & 853 & .373 & 5.67 & 3.58 & 2.09 & 0152 & 0288 & 0149 & 0055 & 4894 \\
\hline 46.7 & -20 & 853 & .373 & 5.92 & 3.58 & 2.34 & 0170 & 0288 & 0167 & 0062 & 5008 \\
\hline 58.0 & -20 & 853 & .373 & 6.20 & 3.58 & 2.62 & 0194 & 0288 & 0191 & 0070 & 5290 \\
\hline 66.5 & -20 & 853 & .373 & 5.20 & 3.58 & 2.62 & 0199 & 0288 & 0196 & 0070 & 5468 \\
\hline 72.0 & -20 & 853 & .373 & 6.35 & .58 & 2.77 & 0201 & 0288 & 0198 & 0074 & 5572 \\
\hline 78.0 & -20 & 853 & .37 & 5.29 & 3.58 & 2.71 & 0199 & 0288 & 0196 & $007 ?$ & 5676 \\
\hline 80. & $\begin{array}{l}-20 \\
-20\end{array}$ & $\begin{array}{r}853 \\
852\end{array}$ & $\begin{array}{r}.373 \\
273\end{array}$ & 6.21 & 3.58 & 2.63 & 0197 & 0288 & 0194 & 0070 & 5709 \\
\hline & & 853 & .37 & 25 & 58 & 2.67 & 0179 & 0288 & 0176 & 0071 & 5974 \\
\hline
\end{tabular}

* last column employed in computer plotting only. 
TABULATION OF SMOOTHED DATA POINTS FOR

ABSORPTION OF SQUND IN AIR VERSUS RELATIVE HUMIDITY

AT -40 DEGREES CENTIGRADE

\begin{tabular}{|c|c|c|c|c|c|c|c|c|c|c|c|}
\hline $\mathrm{R} \bullet \mathrm{H} \bullet$ & TEMP & FREQ & LAMDA & $\begin{array}{c}\text { AIR } \\
+\end{array}$ & & $\begin{array}{l}\text { AIR } \\
O N L Y\end{array}$ & $\begin{array}{l}\text { AIR } \\
\text { ONLY }\end{array}$ & $\begin{array}{l}\text { AIR } \\
\text { CLAS }\end{array}$ & $\begin{array}{l}\text { AIR } \\
\forall O L\end{array}$ & MU & $\begin{array}{l}3000 * \\
\text { LOG }\end{array}$ \\
\hline$P R C T$ & $\begin{array}{l}\text { DEG } \\
\text { CENT }\end{array}$ & CPS & METRS & $\begin{array}{l}\text { WALL } \\
D B / S\end{array}$ & $\begin{array}{l}\text { WALL } \\
D B / S\end{array}$ & $D E / 5$ & $/ M$ & $/ M$ & $/ M$ & & $\mathrm{RH}$ \\
\hline
\end{tabular}

$12345678901234567890^{2} 1234567890^{3} 012345678901234567890123456789012345678$

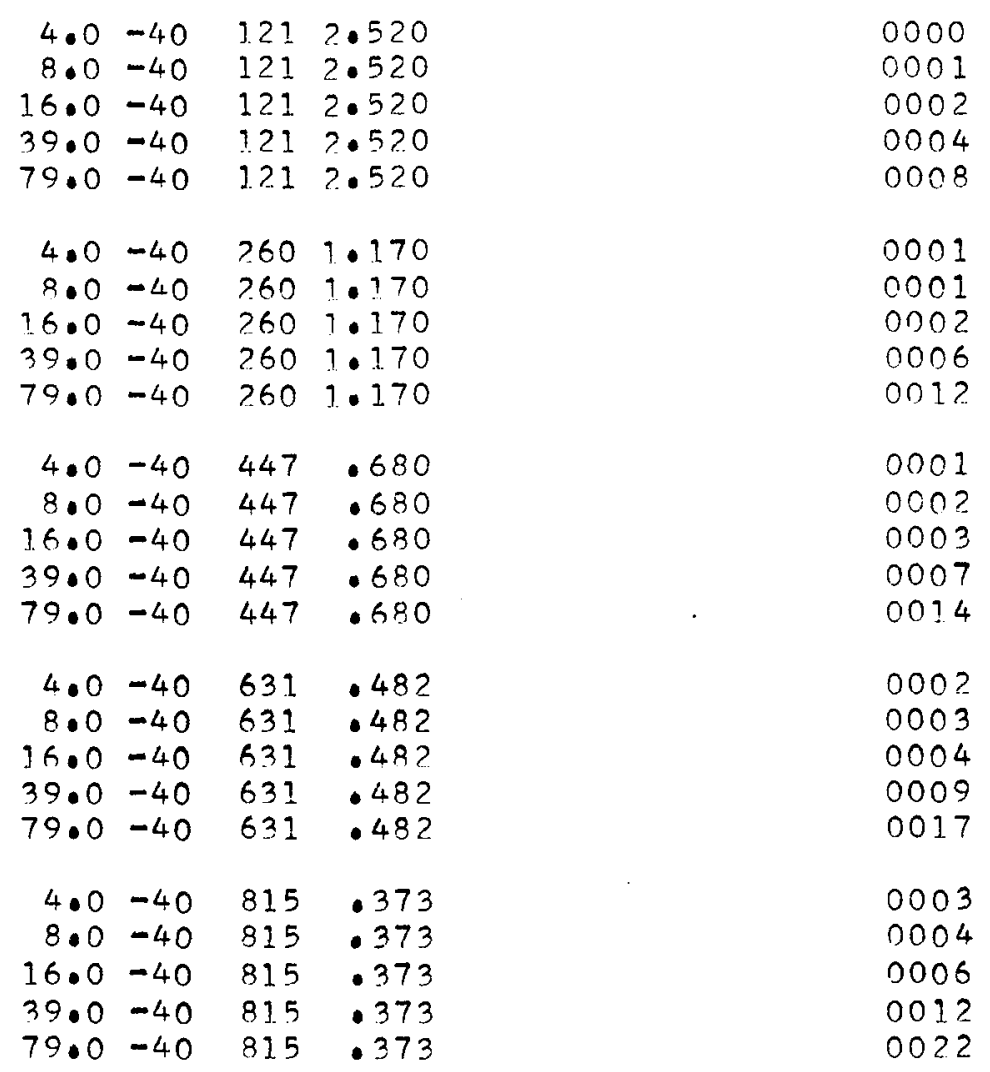

* last column employed in computer plotting only. 
TABLE NO. 12

TABULATION OF ORIGINAL DATA POINTS FOR

ABSORPTION OF SOUND IN OXYGEN VERSUS RELATIVE HUMIDITY AT 20 DEGREES CENTIGRADE

\begin{tabular}{|c|c|c|c|c|c|c|c|c|c|c|c|}
\hline R.H. & TEMP & FREQ & LAMDA & $\begin{array}{r}02 \\
+\end{array}$ & & $\begin{array}{l}02 \\
\text { ONLY }\end{array}$ & $\begin{array}{l}02 \\
\text { ONLY }\end{array}$ & $\begin{array}{l}02 \\
\text { CLAS }\end{array}$ & ${ }^{02}$ & MU & $3000^{*}$ \\
\hline RCT & $\begin{array}{l}\text { DEG } \\
\text { CENT }\end{array}$ & CPS & METRS & $\begin{array}{l}\text { WALL } \\
\mathrm{DB} / \mathrm{S}\end{array}$ & $\begin{array}{l}\text { WALL } \\
\text { DB/S }\end{array}$ & $D B / S$ & $/ M$ & $/ M$ & $/ M$ & & $\begin{array}{l}\text { RUG } \\
\text { RH }\end{array}$ \\
\hline
\end{tabular}

12345678901234567890123456789012345678901234567890123456789012345678

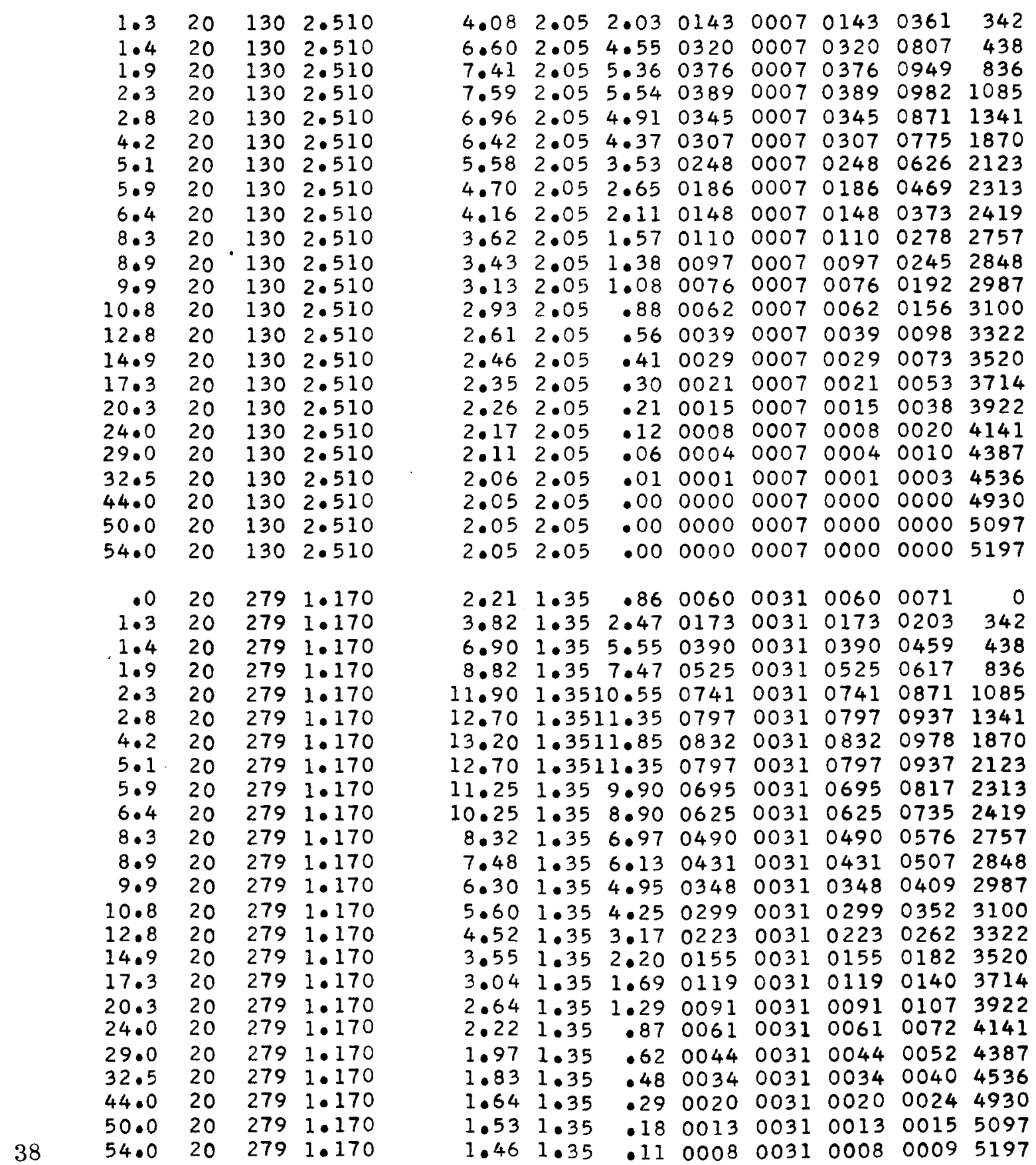

$\approx$ last column employed in computer plotting only. 
TABLE NO• 12

TABULATION OF ORIGINAL DATA POINTS FOR

ABSORPTION OF SOUND IN OXYGEN VERSUS RELATIVE HUMIDITY

\begin{tabular}{|c|c|c|c|c|c|c|c|c|c|c|c|}
\hline $\mathrm{R} \bullet \mathrm{H} \bullet$ & TEMP & FREQ & LAMDA & $\begin{array}{c}\text { AT } 20 \\
02 \\
+\end{array}$ & DEOH & $\begin{array}{l}\text { REES } \\
\text { O2 } \\
\text { ONLY }\end{array}$ & $\begin{array}{l}\text { CENTI } \\
02 \\
\text { ONLY }\end{array}$ & $\begin{array}{l}\text { GRADE } \\
02 \\
\text { CLAS }\end{array}$ & $\stackrel{02}{\mathrm{MOL}}$ & $M U$ & $3000 \%$ \\
\hline PRCT & $\begin{array}{l}\text { DEG } \\
\text { CENT } \\
1\end{array}$ & CPS & $\underset{2}{\operatorname{METRS}}$ & $\begin{array}{l}\text { WALL } \\
D B / S \\
3\end{array}$ & $\begin{array}{l}\text { WALL } \\
D B / S\end{array}$ & $\begin{array}{l}D B / S \\
4\end{array}$ & $/ M$ & $5^{/ M}$ & $/ M$ & 6 & $\begin{array}{l}\mathrm{LUU} \\
\mathrm{RH}\end{array}$ \\
\hline
\end{tabular}

12345678901234567890123456789012345678901234567890123456789012345678

\begin{tabular}{|c|c|c|c|c|c|c|c|c|c|c|c|}
\hline $\begin{array}{l}.0 \\
1.3\end{array}$ & $\begin{array}{l}20 \\
20\end{array}$ & $\begin{array}{l}479 \\
479\end{array}$ & $\begin{array}{l}.680 \\
.680\end{array}$ & $\begin{array}{l}1.94 \\
3.52\end{array}$ & $\begin{array}{l}1.07 \\
1.07\end{array}$ & $\begin{array}{r}.87 \\
2.45\end{array}$ & $\begin{array}{l}0061 \\
0172\end{array}$ & $\begin{array}{l}0092 \\
0092\end{array}$ & $\begin{array}{l}0060 \\
0171\end{array}$ & $\begin{array}{l}0041 \\
0117\end{array}$ & 342 \\
\hline 1.4 & 20 & 479 & & 28 & 1.07 & 6.21 & 0436 & 0092 & 0435 & 0298 & 438 \\
\hline $\begin{array}{l}1 \cdot 9 \\
2 \cdot 3\end{array}$ & $\begin{array}{l}20 \\
20\end{array}$ & $\begin{array}{l}479 \\
479\end{array}$ & $\begin{array}{l}.680 \\
.680\end{array}$ & $\begin{array}{r}9.45 \\
12.40\end{array}$ & $\begin{array}{l}1.07 \\
1.071\end{array}$ & $\begin{array}{l}8.38 \\
1.33\end{array}$ & $\begin{array}{l}0589 \\
0796\end{array}$ & $\begin{array}{l}0092 \\
0092\end{array}$ & $\begin{array}{l}0588 \\
0795\end{array}$ & $\begin{array}{l}0403 \\
0544\end{array}$ & $\begin{array}{r}836 \\
1085\end{array}$ \\
\hline $\begin{array}{l}2 \cdot 8 \\
4 \cdot 2 \\
5 \cdot 1\end{array}$ & $\begin{array}{l}20 \\
20 \\
20\end{array}$ & $\begin{array}{l}479 \\
479 \\
479\end{array}$ & $\begin{array}{l}.680 \\
.680 \\
.680\end{array}$ & $\begin{array}{l}15.70 \\
17.90 \\
20.60\end{array}$ & $\begin{array}{l}1.071 \\
1.071 \\
1.071\end{array}$ & & $\begin{array}{l}1028 \\
1182 \\
1372\end{array}$ & $\begin{array}{l}0092 \\
0092 \\
0092\end{array}$ & $\begin{array}{l}1027 \\
1181 \\
1371\end{array}$ & $\begin{array}{l}0703 \\
0809 \\
0939\end{array}$ & $\begin{array}{l}1341 \\
1870 \\
2123\end{array}$ \\
\hline $\begin{array}{l}5.9 \\
6.4 \\
8.3\end{array}$ & $\begin{array}{l}20 \\
20 \\
20\end{array}$ & $\begin{array}{l}479 \\
479 \\
479\end{array}$ & $\begin{array}{l}.680 \\
.680 \\
.680\end{array}$ & & & 20.33 & $\begin{array}{l}1428 \\
1372 \\
1217\end{array}$ & $\begin{array}{l}0092 \\
0092 \\
0092\end{array}$ & & $\begin{array}{l}0977 \\
0939 \\
0833\end{array}$ & $\begin{array}{l}2313 \\
2419 \\
2757\end{array}$ \\
\hline 8.9 & $\begin{array}{l}20 \\
20 \\
20\end{array}$ & $\begin{array}{l}479 \\
479 \\
479\end{array}$ & $\begin{array}{l}.680 \\
.680 \\
.680\end{array}$ & & & $\begin{array}{l}5.68 \\
3.38 \\
1.73\end{array}$ & $\begin{array}{l}1101 \\
0940 \\
0824\end{array}$ & $\begin{array}{l}0092 \\
0092 \\
0092\end{array}$ & & $\begin{array}{l}0753 \\
0643 \\
0564\end{array}$ & $\begin{array}{l}2848 \\
2987 \\
3100\end{array}$ \\
\hline .8 & 20 & 479 & - 68 & $10 \cdot 30$ & 1.07 & 9.23 & 0648 & 0092 & 0647 & 443 & $\begin{array}{l}3322 \\
3520\end{array}$ \\
\hline 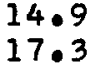 & 20 & $\begin{array}{l}479 \\
479\end{array}$ & & & & & & $\begin{array}{l}0092 \\
0092\end{array}$ & & $\begin{array}{l}0318 \\
0250\end{array}$ & $\begin{array}{l}3520 \\
3714\end{array}$ \\
\hline 1.3 & 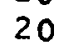 & 479 & .6 & 5.12 & 7 & 4.05 & 0284 & 0092 & 83 & 0194 & 3922 \\
\hline & & 479 & & 8 & 1 & & 0197 & & & 34 & $414 ?$ \\
\hline & 20 & 479 & & & 07 & & 0145 & & & 99 & 4387 \\
\hline & 20 & 479 & - 6 & 72 & 1.07 & 1.65 & 0116 & 0092 & 15 & 0079 & 4536 \\
\hline .0 & 20 & 479 & & 2.22 & 1. & 1.15 & 0 & 2 & 30 & 55 & 4930 \\
\hline 0 & 20 & 479 & .6 & 1.89 & 1.07 & .82 & 58 & 92 & 0 & 39 & 5097 \\
\hline - & 20 & 479 & & 78 & 1.07 & $\bullet 71$ & 50 & 32 & & 34 & 5197 \\
\hline .0 & 0 & 677 & 6 & 3.16 & 1.93 & 1.23 & 0086 & 33 & & 41 & V \\
\hline $1 \cdot 3$ & 20 & 67 & .48 & $4 \cdot 73$ & 1.93 & 2.80 & 0197 & 33 & 95 & 94 & 342 \\
\hline 3 & & 7 & & 72 & 1 & 6.79 & 77 & & & & 438 \\
\hline & & 67 & & & & 07 & 0637 & 83 & 0635 & 0308 & 836 \\
\hline $2 \cdot 3$ & 20 & 677 & .48 & $14 \cdot 10$ & 1. & 17 & 0855 & 0183 & 0853 & 0413 & 1085 \\
\hline 2.8 & $\begin{array}{l}20 \\
20\end{array}$ & $\begin{array}{l}677 \\
677\end{array}$ & $\begin{array}{l}.48 \\
.48\end{array}$ & & & & & & & $\begin{array}{l}0563 \\
0669\end{array}$ & $\begin{array}{l}1341 \\
1870\end{array}$ \\
\hline $5 \cdot 1$ & 20 & 677 & .48 & & & 25.27 & 1775 & 0183 & 1773 & 0859 & 2123 \\
\hline & 20 & 677 & .4 & & & & 2000 & & & 0968 & 2313 \\
\hline - 4 & 20 & 677 & - 48 & 60 & 1 & 67 & 2084 & 0183 & 32 & 09 & 2419 \\
\hline & 20 & 67 & & & & & & & & 1 & 2757 \\
\hline 8 & 20 & 677 & .4 & & & & 1873 & 83 & 71 & 0907 & 2848 \\
\hline .8 & 20 & 677 & .48 & & ? & 17 & 1698 & 0183 & 1696 & 0822 & 2987 \\
\hline & 20 & 677 & & & & & & & & 0726 & 3100 \\
\hline & 20 & 67 & & & & & 1238 & 0 & 36 & 0599 & 3322 \\
\hline & 3 & 67 & & & & & 09 & & 23 & 0447 & 3520 \\
\hline & 20 & 677 & & & & & & & & 0352 & 3714 \\
\hline & $\begin{array}{l}20 \\
20\end{array}$ & $\begin{array}{l}677 \\
677\end{array}$ & $\begin{array}{r}.482 \\
.482\end{array}$ & & & & $\begin{array}{l}0581 \\
0410\end{array}$ & $\begin{array}{l}0183 \\
0183\end{array}$ & $\begin{array}{l}0579 \\
0408\end{array}$ & $\begin{array}{l}0281 \\
0198\end{array}$ & $\begin{array}{l}3922 \\
4141\end{array}$ \\
\hline & 20 & 677 & .48 & 0 & 3 & 4.37 & 0307 & 0183 & 05 & 0148 & 4387 \\
\hline & 20 & 67 & & & & & & & & 0119 & 4536 \\
\hline & 20 & 67 & & & & & 0179 & & & 0086 & 4930 \\
\hline & 20 & 677 & 4 & & & & 0134 & & 32 & 0064 & 509 \\
\hline & & 677 & .482 & & & & 0115 & 0183 & 113 & 0055 & $1=$ \\
\hline
\end{tabular}

* last column employed in computer plotting only. 
TABLE NO. 12

TABULATION OF ORIGINAL DATA POINTS FOR

ABSORPTION OF SOUND IN OXYGEN VERSUS RELATIVE HUMIDITY

\begin{tabular}{|c|c|c|c|c|c|c|c|c|c|c|c|}
\hline$H_{0}$ & & FREQ & LAMDA & $\begin{array}{r}A 1 \\
02 \\
+\end{array}$ & & $\begin{array}{l}\text { ONLY } \\
\text { ONL }\end{array}$ & ONLY & $\begin{array}{l}\text { OL } \\
\text { CLAS }\end{array}$ & 02 & MU & $3000 \%$ \\
\hline$R C T$ & $\begin{array}{l}\text { DEG } \\
\text { CENT }\end{array}$ & CPS & METRS & $\begin{array}{l}\text { WALL } \\
\text { DB/S }\end{array}$ & $\begin{array}{l}\text { WALL } \\
D B / S\end{array}$ & $D B / S$ & $/ M$ & $/ M$ & $/ M$ & & $\begin{array}{l}\text { LOG } \\
R H\end{array}$ \\
\hline
\end{tabular}

12345678901234567890123456789012345678901234567890123456789012345678

$\begin{array}{rrrlrlllllll}.0 & 20 & 874 & .373 & 5.32 & 4.00 & 1.32 & 0093 & 0310 & 0090 & 0034 & 0 \\ 1.3 & 20 & 874 & .373 & 6.86 & 4.00 & 2.86 & 0201 & 0310 & 0198 & 0074 & 342 \\ 1.4 & 20 & 874 & .373 & 10.70 & 4.00 & 6.70 & 0471 & 0310 & 0468 & 0176 & 438 \\ 1.9 & 20 & 874 & .373 & 13.00 & 4.00 & 9.00 & 0632 & 0310 & 0629 & 0236 & 836 \\ 2.3 & 20 & 874 & .373 & 16.30 & 4.0012 .30 & 0864 & 0310 & 0861 & 0323 & 1085 \\ 2.8 & 20 & 874 & .373 & 21.00 & 4.0017 .00 & 1194 & 0310 & 1191 & 0447 & 1341 \\ 4.2 & 20 & 874 & .373 & 24.60 & 4.0020 .60 & 1447 & 0310 & 1444 & 0542 & 1870 \\ 5.1 & 20 & 874 & .373 & 32.70 & 4.0028 .70 & 2016 & 0310 & 2013 & 0756 & 2123 \\ 5.9 & 20 & 874 & .373 & 37.10 & 4.0033 .10 & 2325 & 0310 & 2322 & 0872 & 2313 \\ 6.4 & 20 & 874 & .373 & 39.60 & 4.0035 .60 & 2501 & 0310 & 2498 & 0938 & 2419 \\ 8.3 & 20 & 874 & .373 & 40.30 & 4.0036 .30 & 2550 & 0310 & 2547 & 0956 & 2757 \\ 8.9 & 20 & 874 & .373 & 41.40 & 4.0037 .40 & 2627 & 0310 & 2624 & 0985 & 2848 \\ 9.9 & 20 & 874 & .373 & 36.80 & 4.0032 .80 & 2304 & 0310 & 2301 & 0864 & 2987 \\ 10.8 & 20 & 874 & .373 & 34.60 & 4.0030 .60 & 2149 & 0310 & 2146 & 0805 & 3100 \\ 12.8 & 20 & 874 & .373 & 31.20 & 4.0027 .20 & 1911 & 0310 & 1908 & 0716 & 3322 \\ 14.9 & 20 & 874 & .373 & 25.00 & 4.0021 .00 & 1475 & 0310 & 1472 & 0552 & 3520 \\ 17.3 & 20 & 874 & .373 & 20.80 & 4.0016 .80 & 1180 & 0310 & 1177 & 0442 & 3714 \\ 20.3 & 20 & 874 & .373 & 17.20 & 4.0013 .20 & 0927 & 0310 & 0924 & 0347 & 3922 \\ 24.0 & 20 & 874 & .373 & 13.60 & 4.00 & 9.60 & 0674 & 0310 & 0671 & 0252 & 4141 \\ 29.0 & 20 & 874 & .373 & 11.10 & 4.00 & 7.10 & 0499 & 0310 & 0496 & 0186 & 4387 \\ 32.5 & 20 & 874 & .373 & 9.76 & 4.00 & 5.76 & 0405 & 0310 & 0402 & 0151 & 4536 \\ 44.0 & 20 & 874 & .373 & 7.85 & 4.00 & 3.85 & 0270 & 0310 & 0267 & 0100 & 4930 \\ 50.0 & 20 & 874 & .373 & 7.05 & 4.00 & 3.05 & 0214 & 0310 & 0211 & 0079 & 5097 \\ 54.0 & 20 & 874 & .373 & 6.69 & 4.00 & 2.69 & 0189 & 0310 & 0186 & 0070 & 5197\end{array}$

* last column employed in computer plotting only. 
TABLE NO. 13

TABULATION OF ORIGINAL DATA POINTS FOR

ABSORPTION OF SOUND IN OXYGEN VS CONCENTRATION OF DEUTERIUM OXIDE
R•H. TEMP FREQ LAMDA
02
+
$\begin{array}{lllll}02 & 02 & 02 & 02 & M U\end{array}$
$3000 *$
DEG
WALL WALL
ONLY ONLY CLAS HOL
LOG
PRCT CENT CPS METRS DB/S DB/S DB/S /M /M /M R R

12345678901234567890123456789012345678901234567890123456789012345678

\begin{tabular}{|c|c|c|c|c|c|c|c|c|c|c|c|}
\hline $\begin{array}{l}1.2 \\
1.3\end{array}$ & 20 & $\begin{array}{l}120 \\
130\end{array}$ & 2.510 & $\begin{array}{l}6.73 \\
6.88\end{array}$ & 2.05 & $\begin{array}{l}4.08 \\
4.83\end{array}$ & 0340 & 0007 & 0340 & 0855 & 342 \\
\hline 1.4 & 20 & 130 & $2 \cdot 510$ & 7.08 & 2.05 & 5.03 & 0354 & 0007 & 0354 & & 43 \\
\hline - & 20 & 130 & 2 & 89 & 2.05 & 4.84 & 0341 & 0007 & 0341 & & 528 \\
\hline 1. & 20 & 130 & & 63 & 2.05 & $4 \cdot 58$ & 0 & 07 & 0323 & & $61 ?$ \\
\hline$\bullet$ & 20 & $1=$ & & 6.36 & 2 & $4 \cdot 31$ & 0304 & 007 & 0304 & 5 & 691. \\
\hline & & & & & & 4.04 & 0285 & 007 & 0285 & 7 & 66 \\
\hline & 20 & & & & & $4 \cdot 35$ & 0307 & 007 & 0307 & & \\
\hline & 20 & 130 & & .08 & 2 & 4.03 & 0284 & 0007 & 0284 & 4 & 02 \\
\hline & 20 & 130 & & & & 3.58 & $0 \leq 22$ & 007 & 0252 & & - \\
\hline - & 20 & 13 & & 2.03 & 2.05 & 3.03 & 0214 & 007 & 0214 & & 835 \\
\hline & & & & & & & 0200 & 1007 & 0200 & & 90 \\
\hline & 20 & 130 & & 43 & & 2.38 & 0168 & 0007 & 016 & 3 & 34 \\
\hline • & 20 & 130 & & 12 & 2 & 1.97 & 9 & 0007 & 0127 & & 2290 \\
\hline$\bullet$ & 20 & 130 & 2 & 3.31 & 2.05 & 1.26 & 0039 & 0007 & 0089 & 4 & 255 \\
\hline & 20 & 130 & & 2.95 & & .90 & 0063 & 0007 & 0063 & 58 & 274 \\
\hline & 20 & 130 & & 78 & 2. & .73 & 0051. & 0007 & 0051 & & 311 \\
\hline & 20 & 13 & & & 2 & .44 & 003 & 7 & 0031 & 8 & 3438 \\
\hline & 20 & 13 & & 2.34 & 2 & . 29 & 0020 & 0007 & 0020 & 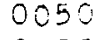 & 376 \\
\hline & 20 & 130 & & 2.24 & 2. & .19 & 0013 & 0007 & 0013 & & 405 \\
\hline & 20 & 130 & & & 2. & .14 & 0010 & 0007 & 0010 & 25 & 4.37 \\
\hline & 20 & 13 & & & & .12 & 0008 & 0007 & 0008 & 0 & 4515 \\
\hline & 20 & 130 & & 1 & 2.05 & .06 & 0004 & 7 & 0004 & 0 & 4705 \\
\hline & 20 & 13 & & 2.10 & 2. & .05 & 0004 & 0007 & 0004 & 10 & 4900 \\
\hline & 20 & 13 & & 2 & 2 & .04 & 0003 & 0007 & 0003 & & 501 \\
\hline & 20 & 27 & & & & 1.07 & 0075 & 0031 & 0075 & & -299 \\
\hline & 20 & 279 & & & & 6.08 & 0428 & 0031 & 0428 & 2 & 238 \\
\hline 1. & 20 & 27 & & 8.40 & 5 & 7.05 & 0497 & 0031 & 0497 & 583 & 342 \\
\hline , & 20 & 279 & 1 & 9.01 & 5 & 7.66 & 0540 & 0031 & 0540 & 3 & 438 \\
\hline & 20 & 27 & & 9. & & 8.0 & 0569 & & & & 52 \\
\hline & 20 & 27 & & & & & 05 & & & & 612 \\
\hline & 20 & 27 & & & & & 0700 & 0031 & 0700 & 320 & 691 \\
\hline 1. & 20 & 27 & & 10 & & 11 。 & 0828 & 0031 & 0328 & 970 & 756 \\
\hline 1. & 20 & 279 & & 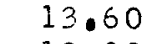 & & 2 & 0863 & 0031 & 0863 & 1 & 83 \\
\hline & 20 & 27 & & & & & 0877 & 31 & & & 1027 \\
\hline & 20 & 2.79 & & & & & 0856 & 0031 & 0856 & 203 & 1194 \\
\hline & 20 & 279 & & & $1 \bullet$ & 11. & 0800 & 0031 & 0800 & 0938 & 1838 \\
\hline & 20 & 279 & & & & & 0779 & 003.1 & 0779 & 913 & 1900 \\
\hline & 20 & 279 & & & & 8 & 0620 & 0031 & 0620 & 727 & 2044 \\
\hline & 20 & 279 & & & & 6.88 & 0485 & 0031 & 35 & 8 & 2290 \\
\hline & 20 & 279 & & & & & 0391 & 31 & 0391 & 458 & 2554 \\
\hline & 20 & 279 & & & . & $4 \cdot 16$ & 0293 & 0031 & 0293 & 0343 & 2741 \\
\hline & 20 & 279 & & 4 & & 3. & 0221 & 0031 & 022.1 & 259 & 3112 \\
\hline & 20 & 279 & & & & & 0154 & 0031 & & 180 & 343 \\
\hline & 20 & 279 & 0.17 & & & & 0117 & 0031 & 0117 & 0137 & 3766 \\
\hline & 20 & 279 & & & & 1.1 & 0078 & 0031 & 0078 & $\cos 1$ & 4051 \\
\hline & 20 & 279 & & & & .80 & 0056 & 0031 & 0056 & 066 & 4378 \\
\hline & 20 & 279 & & & & & 0047 & 003 & 0047 & & 4515 \\
\hline & 20 & 279 & & & & 5 & 0039 & 0031 & 0039 & 46 & \\
\hline & 20 & 79 & 17 & & & & & 0031 & 0032 & J) 38 & 110 \\
\hline
\end{tabular}

* last column employed in computer plotting only. 
TABLE NO. 13

TABULATION OF ORIGINAL DATA POINTS FOR

ABSORPTION OF SOUND IN OXYGEN VS CONCENTRATION OF DEUTERIUM OXIDE

\begin{tabular}{|c|c|c|c|c|c|c|c|c|c|c|c|}
\hline - $H_{0}$ & FMP & FREQ & LAMDA & $\begin{array}{r}02 \\
+\end{array}$ & & $\begin{array}{l}02 \\
\text { ONLY }\end{array}$ & $\begin{array}{l}02 \\
\text { ONLY }\end{array}$ & $\begin{array}{l}02 \\
\text { CLAS }\end{array}$ & 02 & MU & $\begin{array}{l}3000^{*} \\
\text { LOG }\end{array}$ \\
\hline & $D E G$ & & & WALL & WALL & & & & & & $\mathrm{RH}$ \\
\hline$R C T$ & CENT & CPS & METRS & $D B / S$ & $D B / S$ & $D B / S$ & $/ M$ & $/ M$ & $/ M$ & & \\
\hline . 1 & 20 & 479 & & 2.37 & I. 35 & 1.02 & 0072 & 0092 & 0071 & $0048-$ & 999 \\
\hline $\begin{array}{l}1 \cdot 2 \\
1 \cdot 3\end{array}$ & $\begin{array}{l}20 \\
20\end{array}$ & $\begin{array}{l}479 \\
479\end{array}$ & $\begin{array}{l}.680 \\
.680\end{array}$ & $\begin{array}{l}7.60 \\
9.23\end{array}$ & $\begin{array}{l}1.35 \\
1.35\end{array}$ & $\begin{array}{l}6.25 \\
7.88\end{array}$ & $\begin{array}{l}0440 \\
0555\end{array}$ & $\begin{array}{l}0092 \\
0092\end{array}$ & $\begin{array}{l}0439 \\
0554\end{array}$ & $\begin{array}{l}0300 \\
0378\end{array}$ & $\begin{array}{l}238 \\
342\end{array}$ \\
\hline 1.4 & 20 & 479 & .680 & 10.20 & 1.35 & 8.85 & 0624 & 0092 & 0623 & 0425 & 438 \\
\hline 1.5 & 20 & 479 & & .20 & 1.35 & 9.85 & 0694 & 0092 & 0693 & 0473 & 528 \\
\hline $1 \cdot 6$ & 20 & 479 & & 2.70 & 1.351 & 11.35 & 0800 & 0092 & 0799 & 0545 & 612 \\
\hline $1: 7$ & 20 & 479 & & 14.00 & 1.351 & 12.65 & 0891 & 0092 & 0890 & 0608 & 691 \\
\hline & 20 & 479 & & 17.30 & 1.351 & L 5.95 & $\begin{array}{l}0994 \\
1124\end{array}$ & $\begin{array}{l}0092 \\
0092\end{array}$ & $\begin{array}{l}0993 \\
1123\end{array}$ & $\begin{array}{l}0.678 \\
0767\end{array}$ & $\begin{array}{l}166 \\
836\end{array}$ \\
\hline & 20 & & & & & & & & & & \\
\hline 2.5 & 20 & 479 & .6 & 0.80 & 1.351 & 9.45 & 1371 & 0092 & 1370 & 0935 & 1194 \\
\hline $4 \cdot 1$ & 20 & 479 & & 20 & 1 . & 35 & 1399 & 0092 & 1398 & 0954 & 1838 \\
\hline $\begin{array}{l}4 \cdot 3 \\
4 \cdot 8\end{array}$ & $\begin{array}{l}20 \\
20\end{array}$ & $\begin{array}{l}479 \\
479\end{array}$ & & $\begin{array}{l}21.50 \\
20.80\end{array}$ & & $\begin{array}{l}0.15 \\
9.45\end{array}$ & $\begin{array}{l}1420 \\
1371\end{array}$ & $\begin{array}{l}0092 \\
0092\end{array}$ & $\begin{array}{l}1419 \\
1370\end{array}$ & $\begin{array}{l}0969 \\
0935\end{array}$ & $\begin{array}{l}1000 \\
2044\end{array}$ \\
\hline 5.8 & 20 & 479 & .6 & 18.70 & 1.351 & .7 .35 & 1223 & 0092 & 1222 & 0334 & 2290 \\
\hline $7 \cdot 1$ & 20 & 479 & & 15.60 & 1.351 & $4 \cdot 25$ & 1004 & 0092 & 1003 & 0685 & 2554 \\
\hline $8 \cdot 2$ & 20 & 479 & & 13.25 & & 1.90 & 0839 & 0092 & 0838 & 0572 & 2741 \\
\hline 1.9 & 20 & 479 & & 1.0 .70 & & 9.35 & 0659 & 0092 & 0658 & 49 & 3112 \\
\hline 1 & 20 & 47 & - & 07 & 35 & 6 & 4 & 2 & 04 & 3 & 3438 \\
\hline 18.0 & 20 & 479 & - 6 & 6.52 & ]. .35 & 5. & 0364 & 0092 & 0363 & 48 & 3766 \\
\hline & 20 & 4 & & 4.85 & 1.35 & 3.50 & 0247 & 0092 & 0246 & & 405 \\
\hline 8 & 20 & 479 & & 3.98 & 1.35 & 2.63 & 0185 & 0092 & 0184 & & 437 \\
\hline .0 & 20 & 479 & & 3.60 & 35 & 2.25 & 0159 & 0092 & 0158 & 08 & 4515 \\
\hline 37.0 & 20 & 479 & .6 & 3.24 & 1.35 & 2.89 & 0133 & 0092 & 0132 & 0090 & 4705 \\
\hline 3.0 & 20 & 479 & & 3.08 & 1.35 & 1.73 & 0122 & 0092 & 0121 & 0083 & 4900 \\
\hline & 20 & 479 & & & & & 0111 & 0092 & 0110 & 75 & 016 \\
\hline 1 & 20 & 677 & & 69 & & 1.76 & 0124 & Ci183 & 0122 & $59-$ & -299 \\
\hline 1.2 & 20 & 677 & .2 & 9.08 & 93 & $7 \cdot 15$ & 0504 & 0183 & 0502 & 0242 & 238 \\
\hline 1.3 & 20 & 677 & .4 & 10.80 & 1.93 & 8.87 & 0625 & 0183 & 0623 & 0301 & 342 \\
\hline 1.4 & 20 & 677 & .2 & 1.85 & 1.93 & 9.92 & 0699 & 0183 & 0697 & 37 & 438 \\
\hline 1.5 & 20 & 677 & & 10 & 1.93 & 1.17 & 0787 & 0183 & 0785 & 79 & 28 \\
\hline 1.6 & 20 & 677 & & 70 & & 77 & 0900 & 83 & 0898 & & 612 \\
\hline 1.7 & 20 & 6 & & 40 & & 7 & 1020 & 83 & 1018 & 92 & 691 \\
\hline 1.8 & 20 & 677 & & .20 & 093 & 6.27 & 1146 & 0183 & 1144 & 0553 & 766 \\
\hline 1.9 & 20 & 677 & .4 & 0.70 & 1.93 & 8.77 & 1323 & 0183 & 1321 & 0638 & 836 \\
\hline 2.2 & 20 & 6 & & 70 & & 77 & 1534 & 0183 & 1532 & 40 & 2 \\
\hline & 20 & 677 & & & & 17 & 1774 & 83 & 1772 & & 94 \\
\hline $4 \cdot 1$ & 20 & 677 & .4 & 28 & 1.9 & 7 & 1900 & 0183 & 1898 & 17 & 1838 \\
\hline $4 \cdot 3$ & 20 & 677 & & 29.80 & - & 27.87 & 1964 & 0183 & 1962 & 0948 & 1900 \\
\hline $4 \cdot 8$ & 20 & 677 & .48 & 31.70 & .93 & 9.77 & 2098 & 0183 & 2096 & 1012 & 2044 \\
\hline $5 \cdot 8$ & 20 & 677 & .48 & 1.30 & 1.93 & 9.37 & 2070 & 0183 & 2068 & 0999 & 2290 \\
\hline $7 \cdot 1$ & 20 & $6^{\circ}$ & & 90 & 1.9 & .5 .97 & 1830 & 0183 & 1828 & 0883 & 2554 \\
\hline 8 & 20 & $6^{\circ}$ & & 40 & 1 & 2.47 & 1583 & 0183 & 1581 & 64 & 27 \\
\hline 10.9 & 20 & 677 & .482 & 20.40 & 1.93 & 8.47 & 1301 & 0183 & 1299 & 0627 & 3112 \\
\hline 14.0 & 20 & 677 & .48 & 10 & - & 4.17 & 0998 & 0183 & 0996 & 0481 & 3438 \\
\hline 0 & 20 & 677 & & 12.90 & & & 0773 & 0183 & 0771. & 0372 & 3766 \\
\hline 22.4 & 20 & 677 & & & 1.93 & 7.9 & 0558 & 0183 & 0556 & & 4051 \\
\hline . 8 & 20 & 677 & .4 & 8.15 & 1.93 & 6.22 & 0438 & 0133 & 0436 & () 211 & 4378 \\
\hline & 20 & 677 & 14 & 43 & 1.93 & 50 & 0388 & 0183 & 0386 & 0186 & 4524 \\
\hline & 20 & 677 & .482 & 64 & 93 & 1 & 0332 & 0183 & 0330 & 0159 & 4705 \\
\hline 43.0 & 20 & 677 & .482 & 6.38 & 1.93 & 4.45 & 0314 & 0183 & 0312 & 0151 & 4900 \\
\hline .0 & 20 & 677 & .482 & 6.05 & 1.93 & $4 \cdot 12$ & 0290 & 0183 & 0288 & 0139 & 501 \\
\hline
\end{tabular}

* last column employed in computer plotting only. 
TABLE NO, 13

TABULATION OF ORIGINAL DATA POINTS FOR

ABSORPTION OF SOUND IN OXYGEN VS CONCENTRATION $\odot F$ DEUTERIUM OXIDE AT 20 DEGREES CENTIGRADE

\begin{tabular}{|c|c|c|c|c|c|c|c|c|c|c|c|}
\hline $\mathrm{Q} . \mathrm{H}$. & TEMP & FREQ & LAMDA & $\begin{array}{r}02 \\
+\end{array}$ & & $\begin{array}{l}02 \\
\text { ONLY }\end{array}$ & $\begin{array}{l}02 \\
\text { ONLY }\end{array}$ & $\begin{array}{l}02 \\
\text { CLAS }\end{array}$ & 02 & MU & $3000 \%$ \\
\hline $\mathrm{S} R \mathrm{PCT}$ & $\begin{array}{l}\text { DEG } \\
\text { CENT } \\
1\end{array}$ & CPS & $\frac{\text { METRS }}{2}$ & $\begin{array}{l}\text { WALL } \\
D B / S \\
3\end{array}$ & $\begin{array}{l}\text { WALL } \\
D B / S\end{array}$ & $\begin{array}{l}D B / S \\
4\end{array}$ & $/ M$ & ${ }_{5} / M$ & $/ M$ & 6 & $\begin{array}{l}\text { LUG } \\
\text { RH }\end{array}$ \\
\hline
\end{tabular}

12345678901234567890123456789012345678901234567890123456789012345678

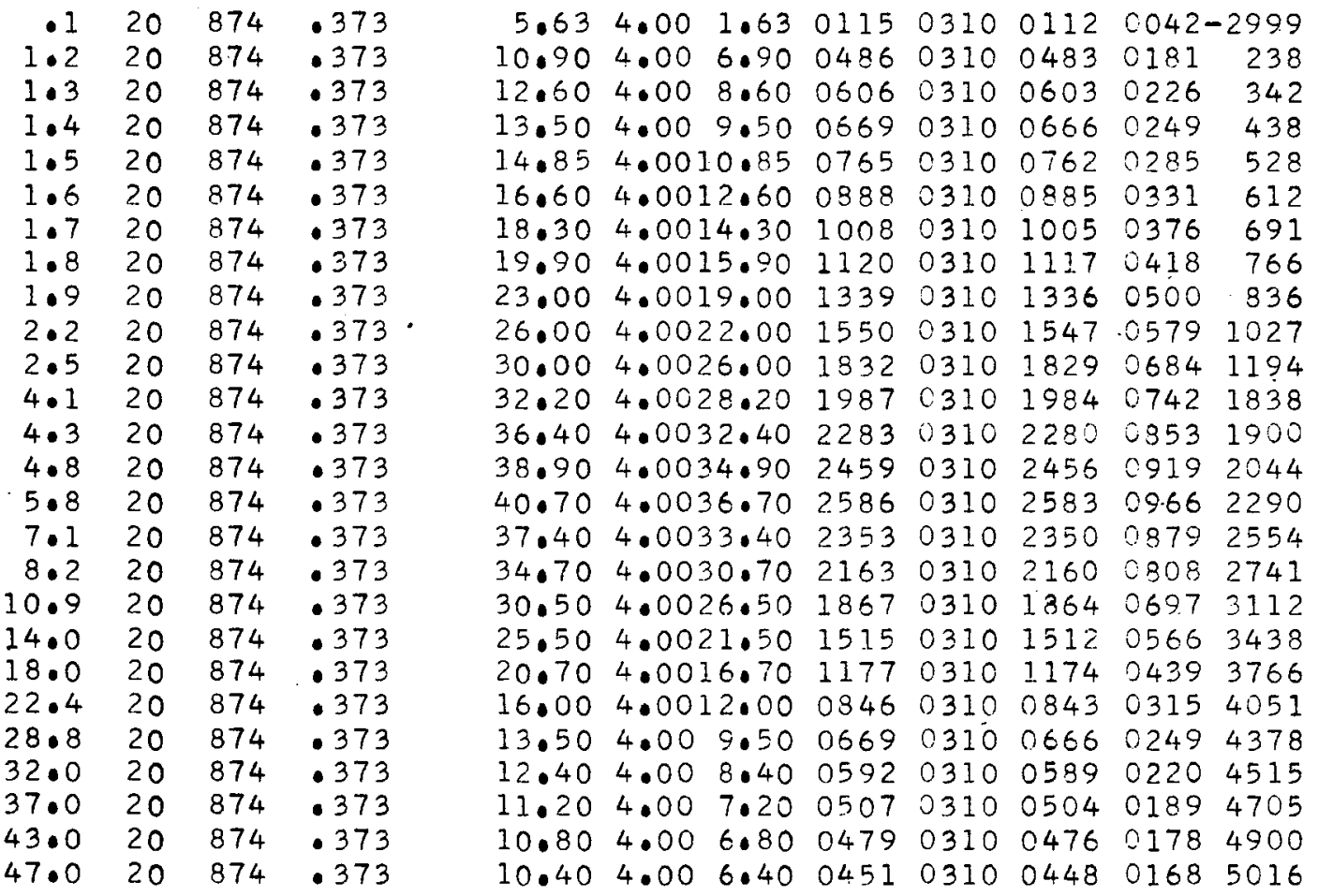

* last column employed in computer plotting only. 


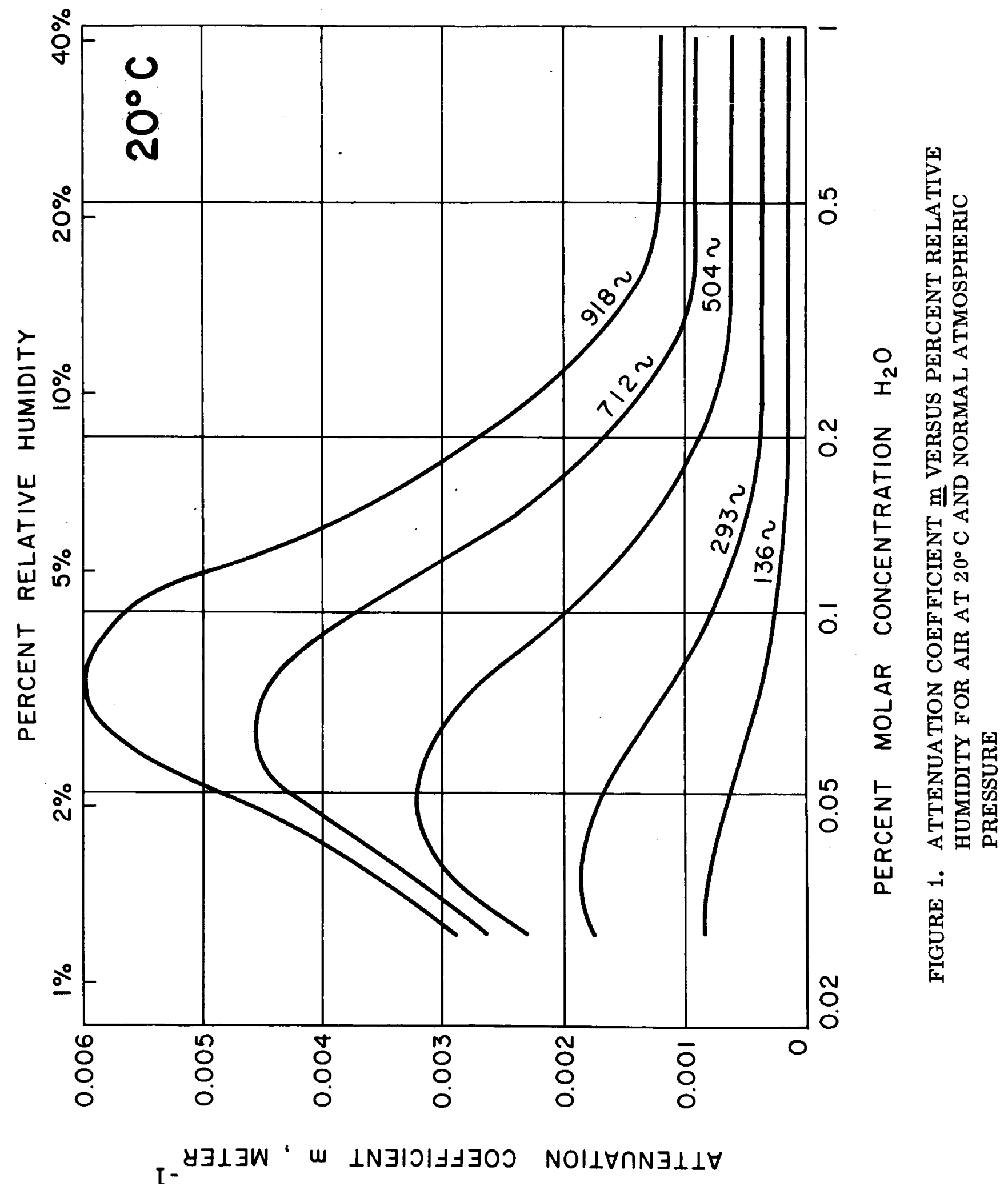




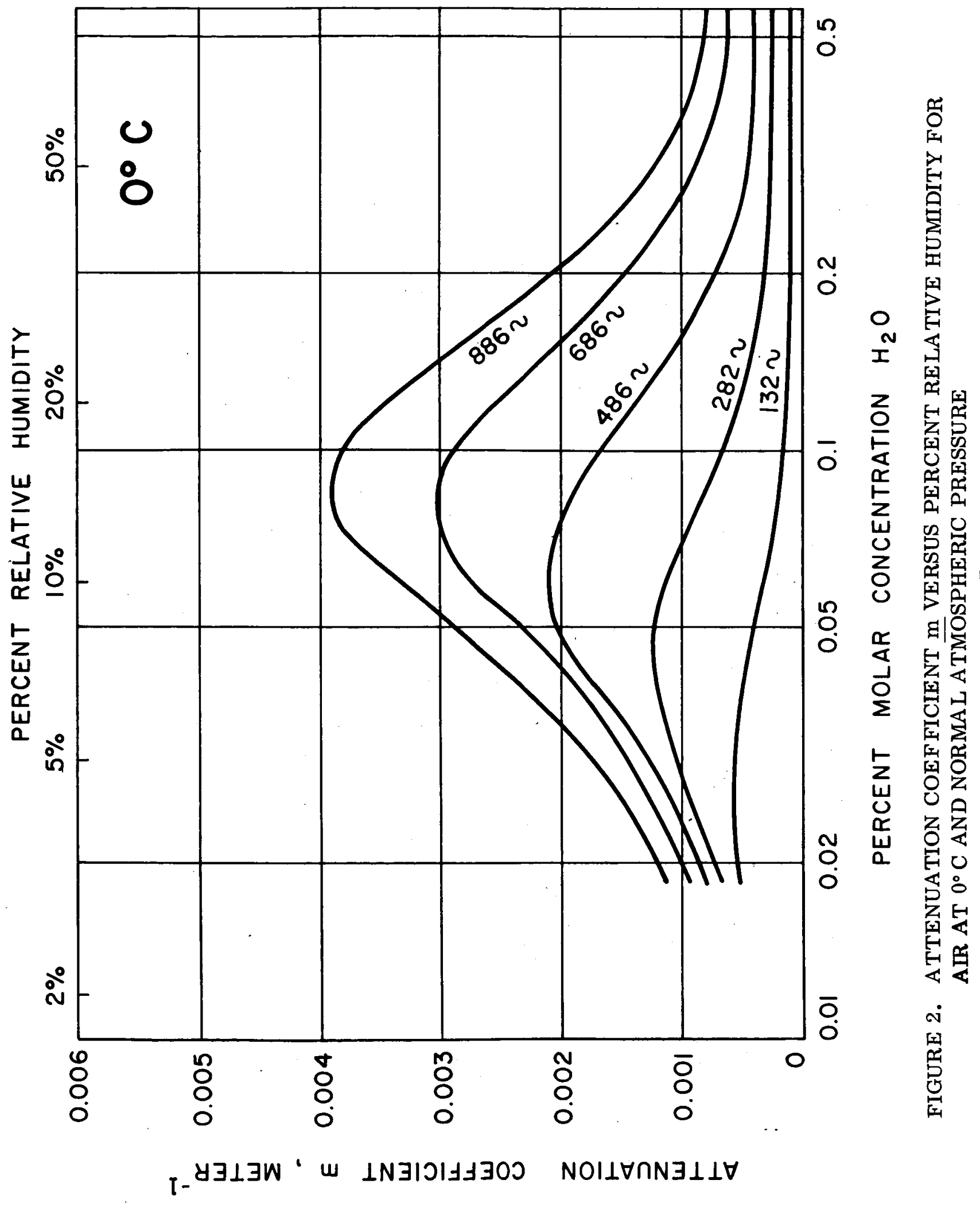




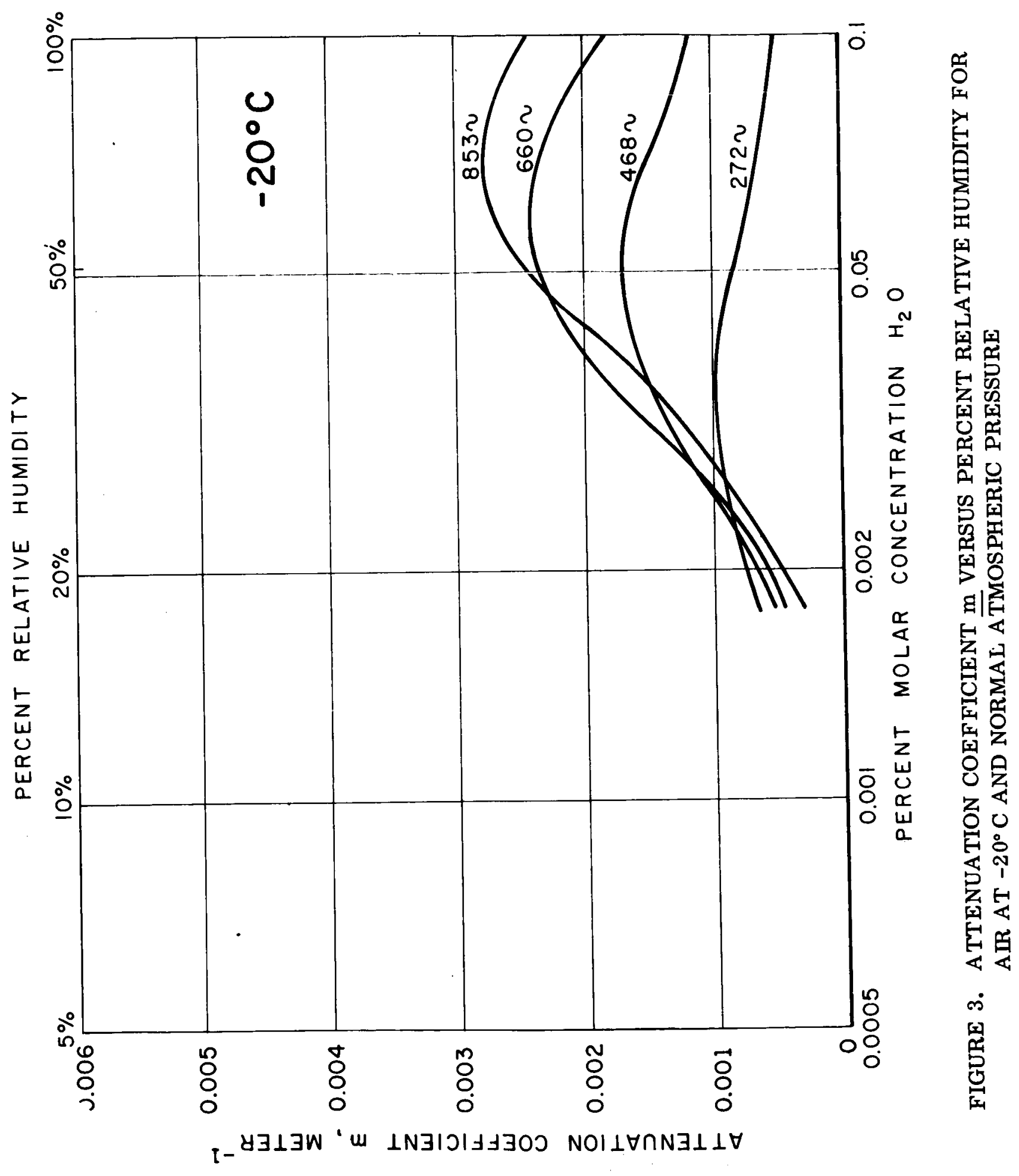




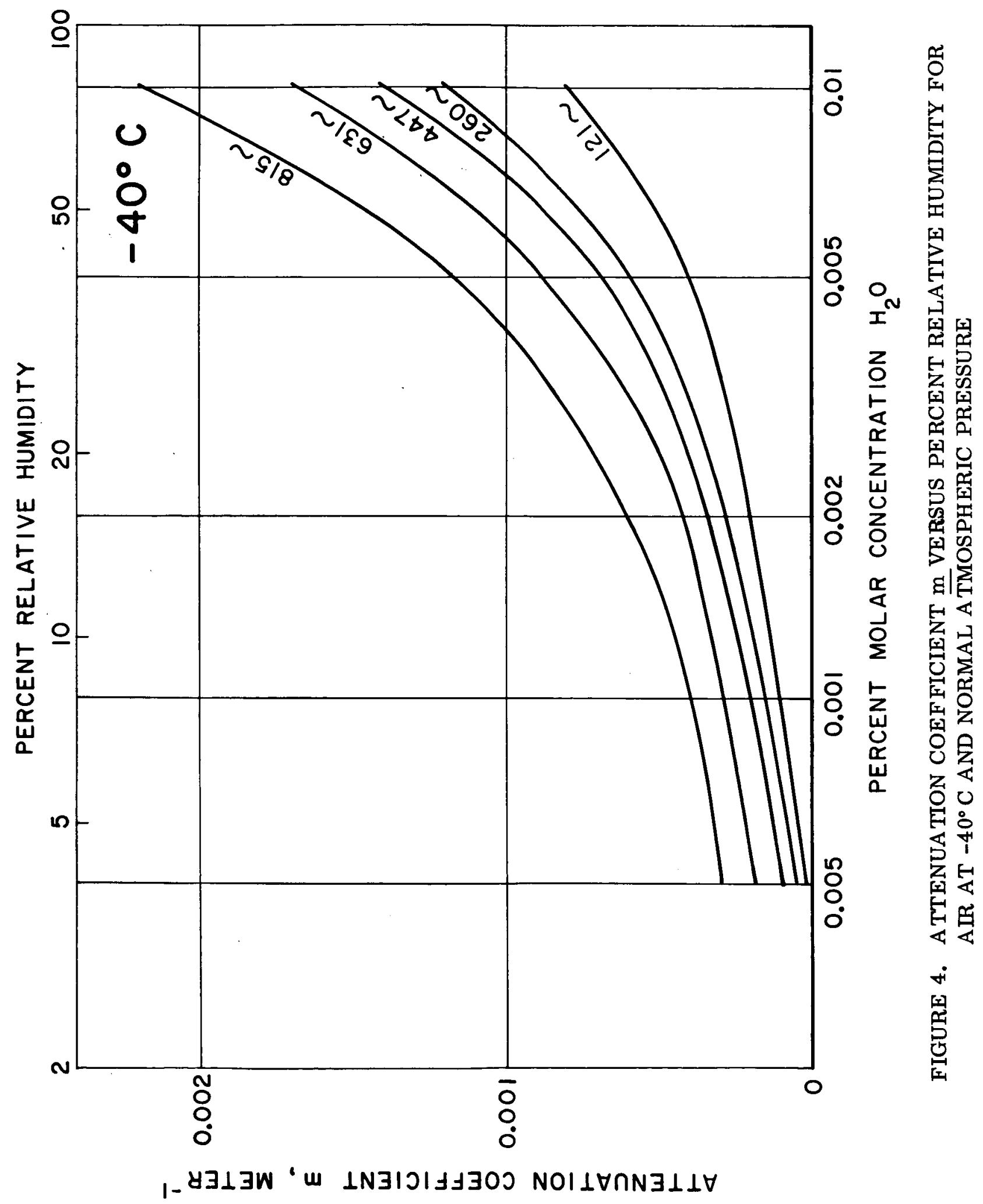




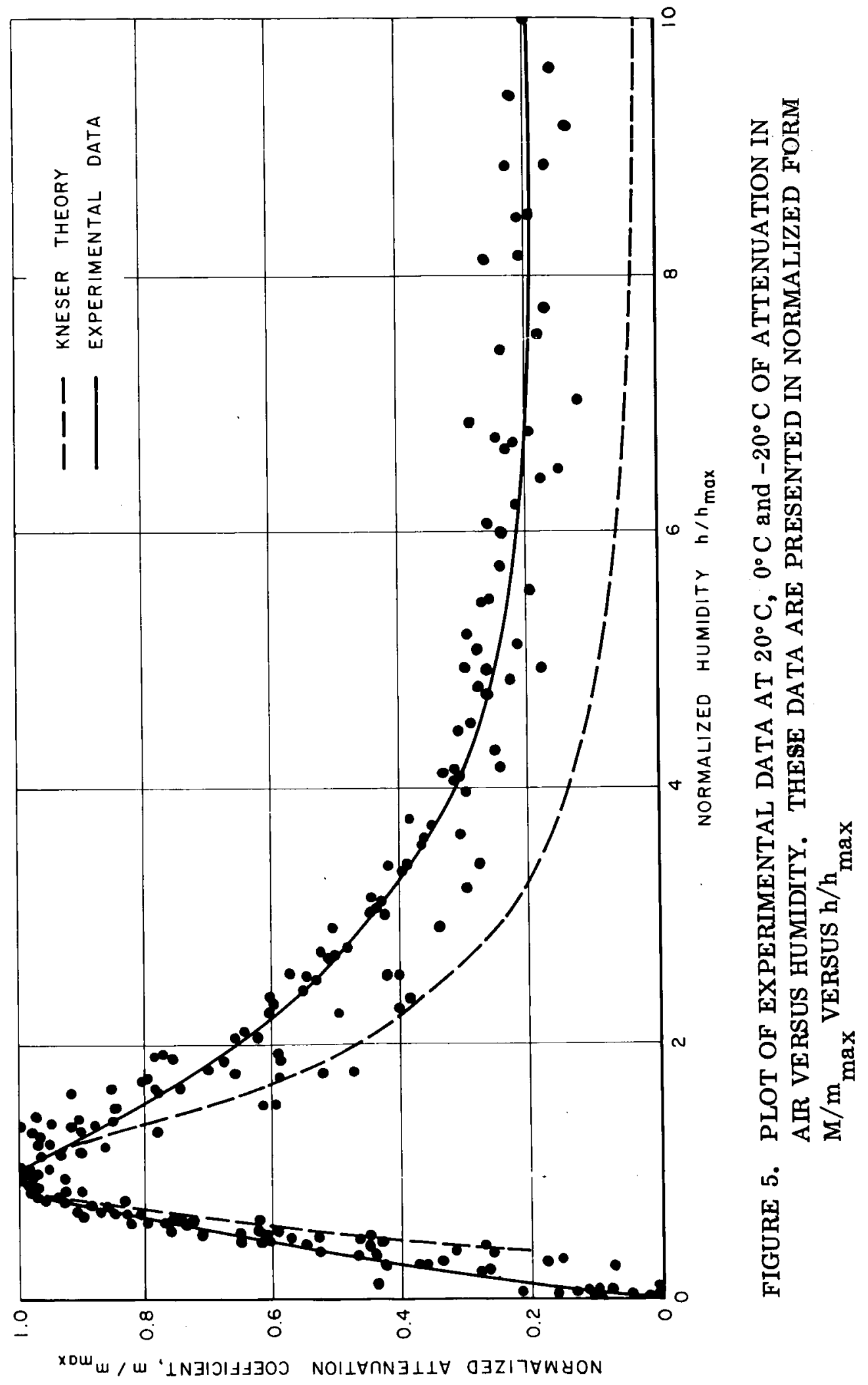




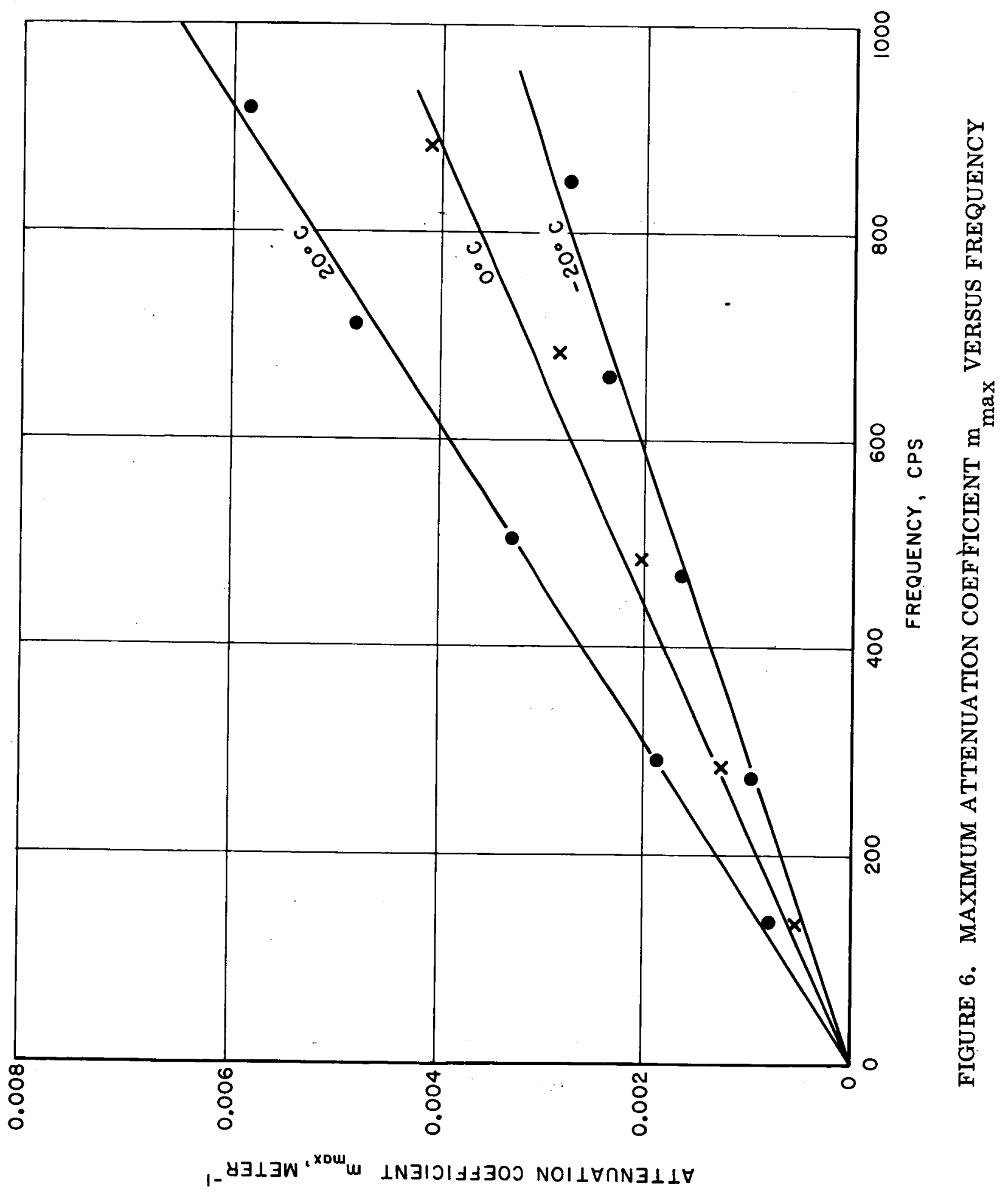




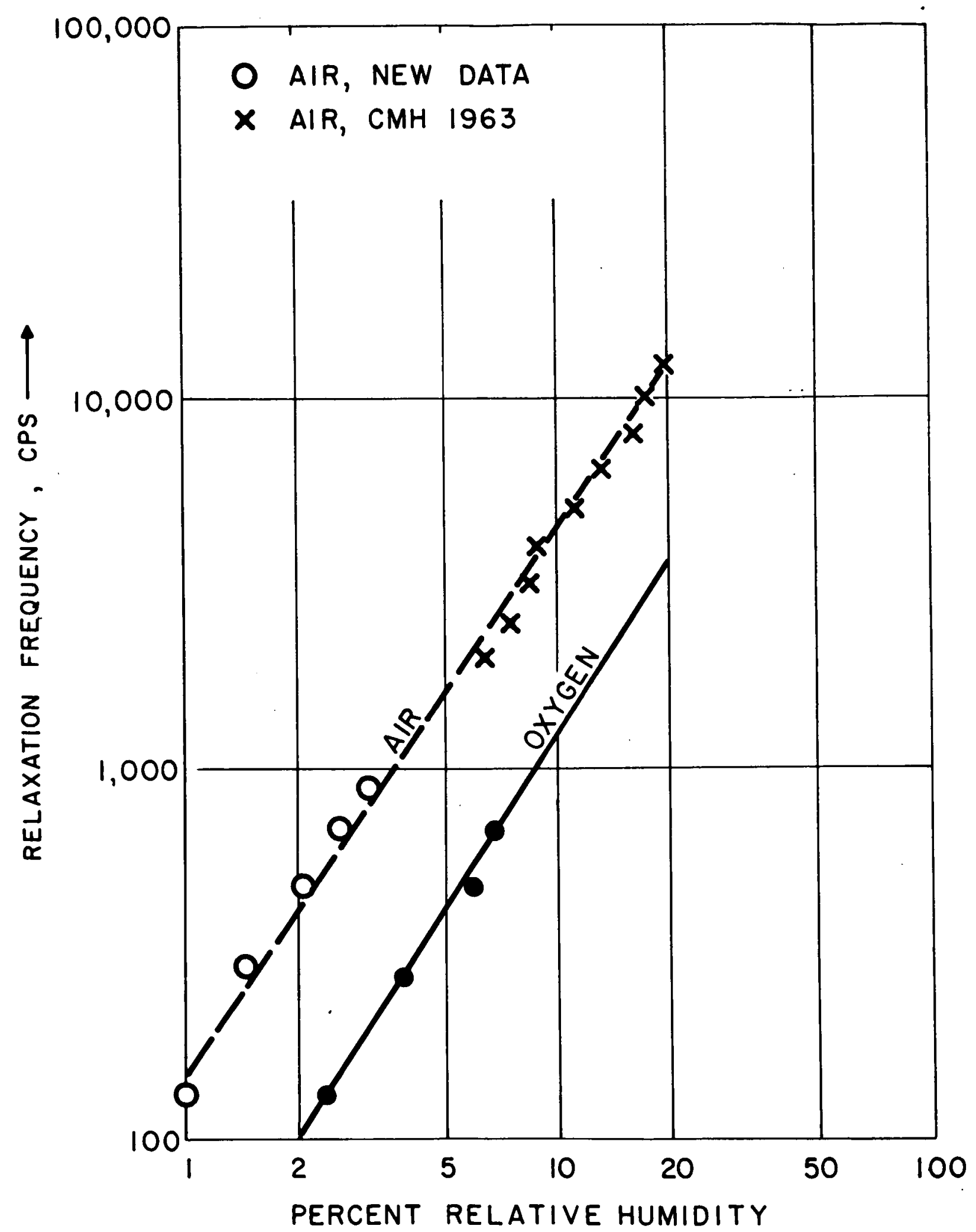

FIGURE 7. RELAXATION FREQUENCY PLOTTED AS A FUNCTION OF h, THE PERCENT MOLAR CONCENTRATION OF WATER VAPOR IN AIR 


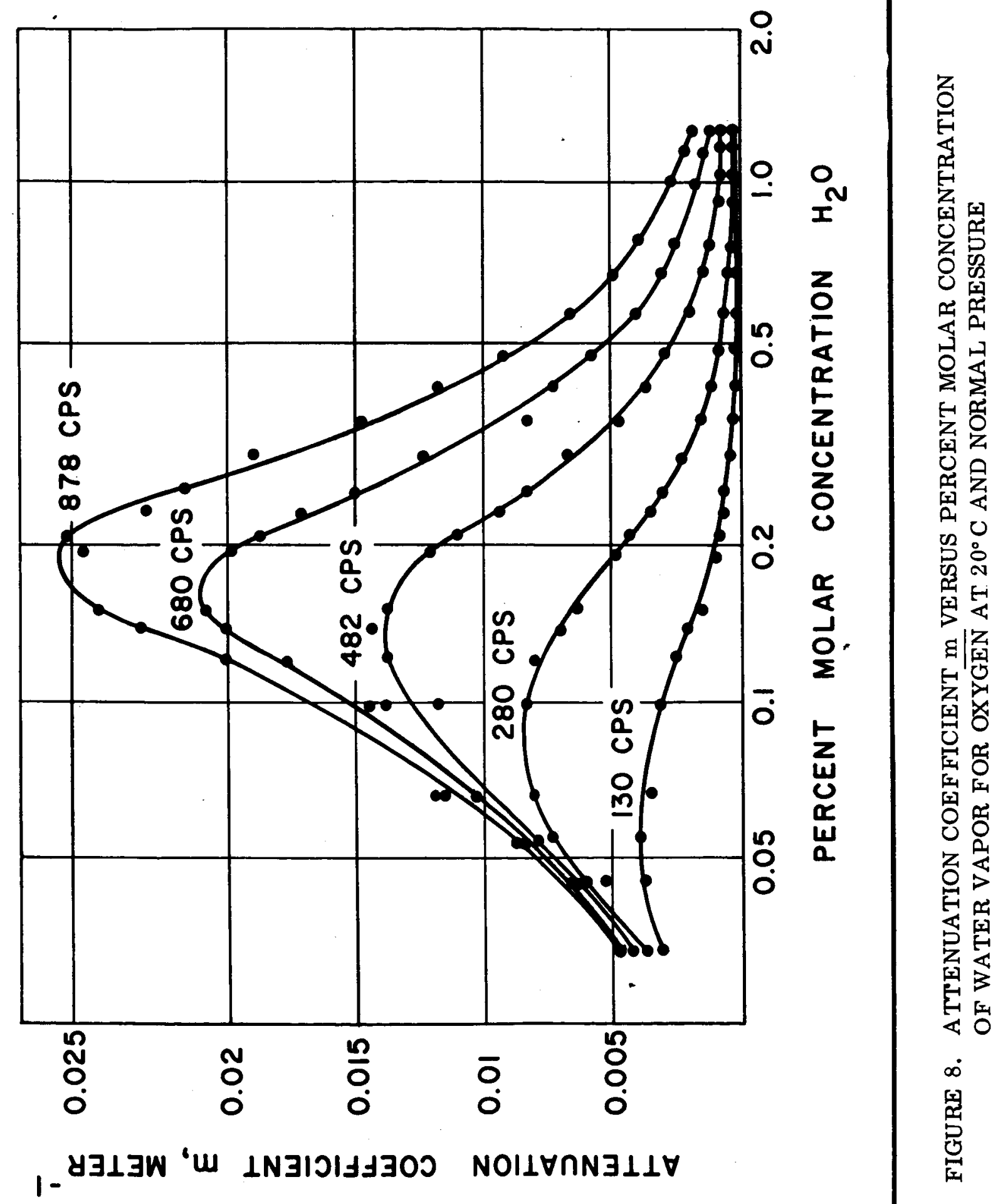




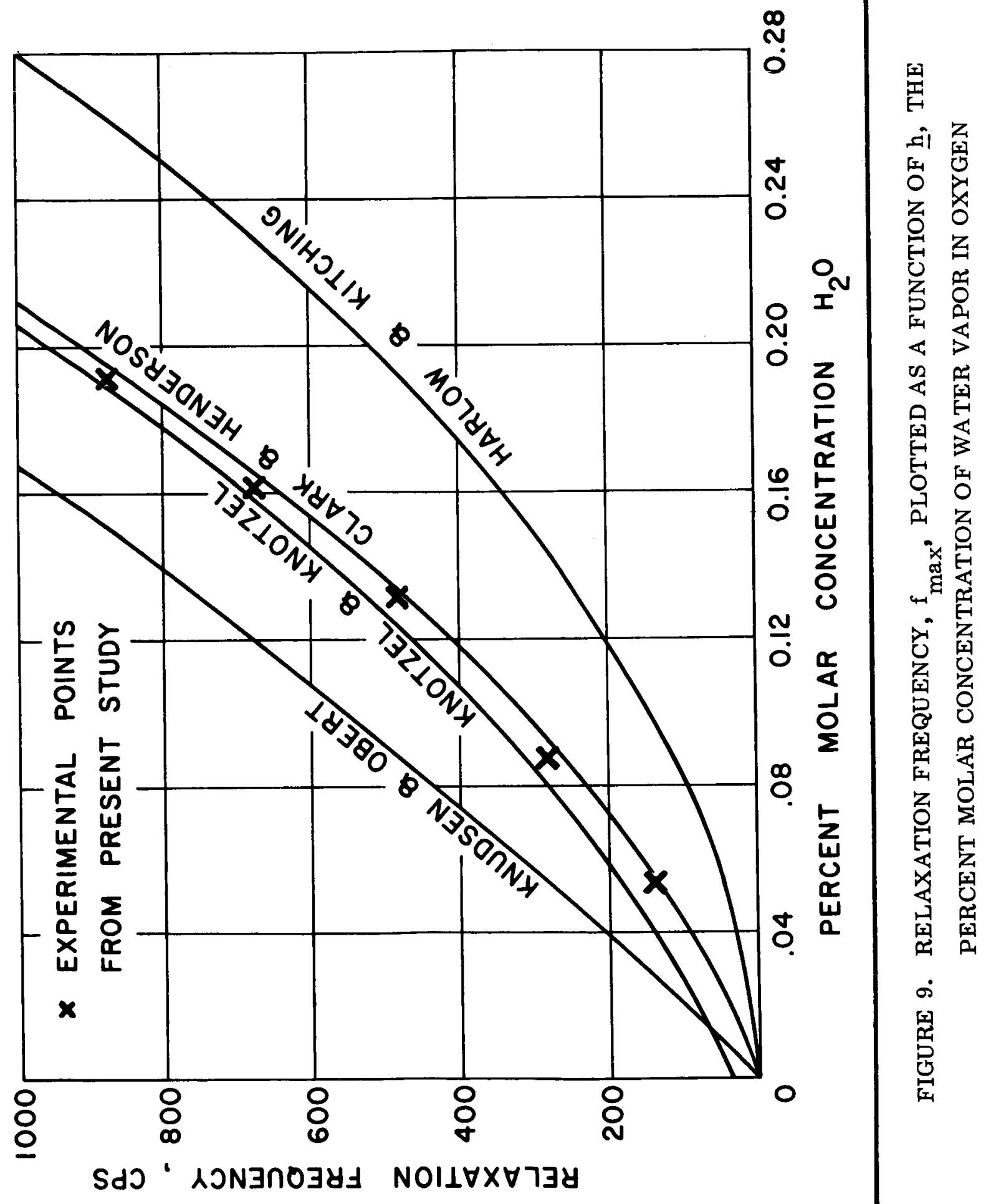




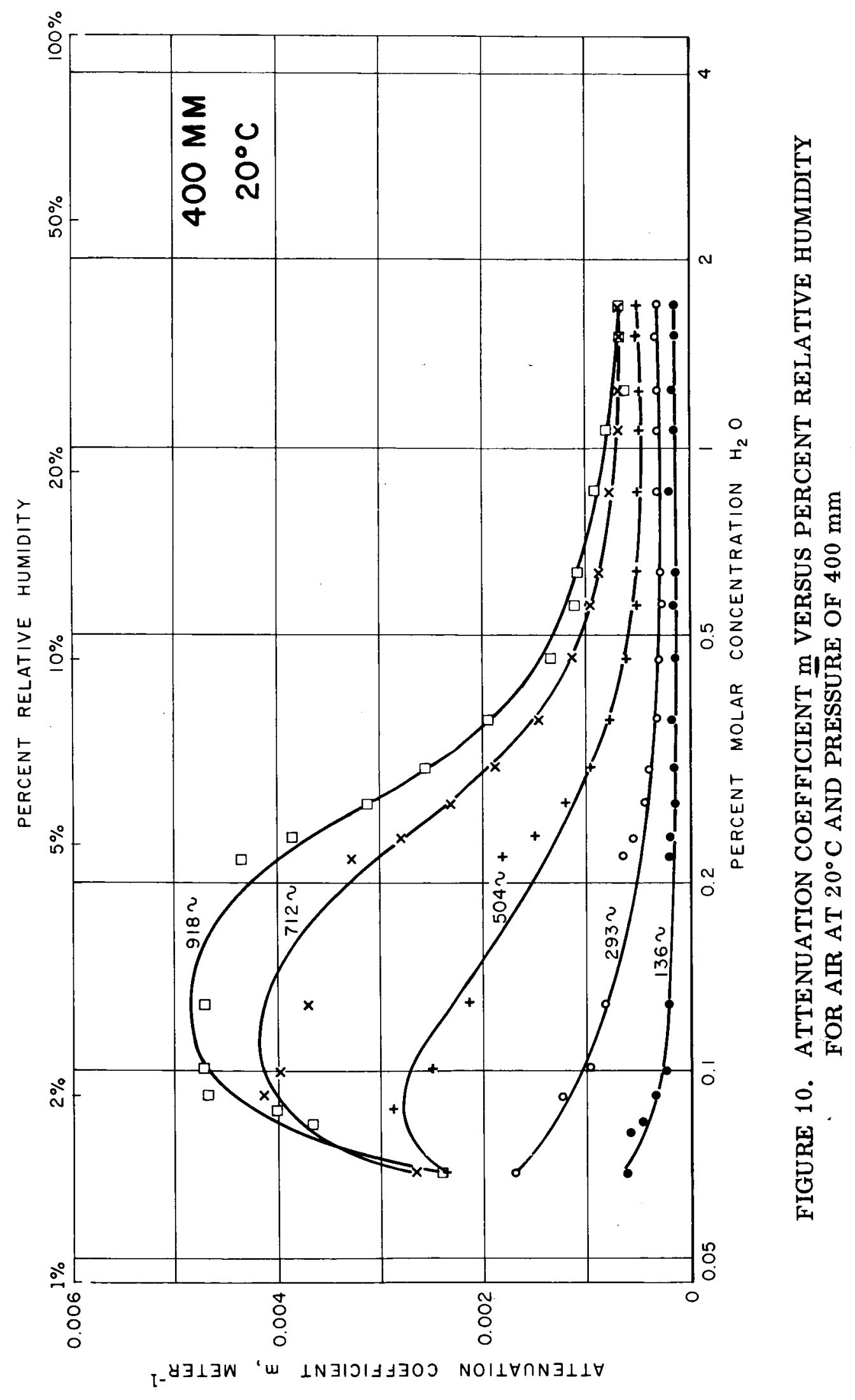




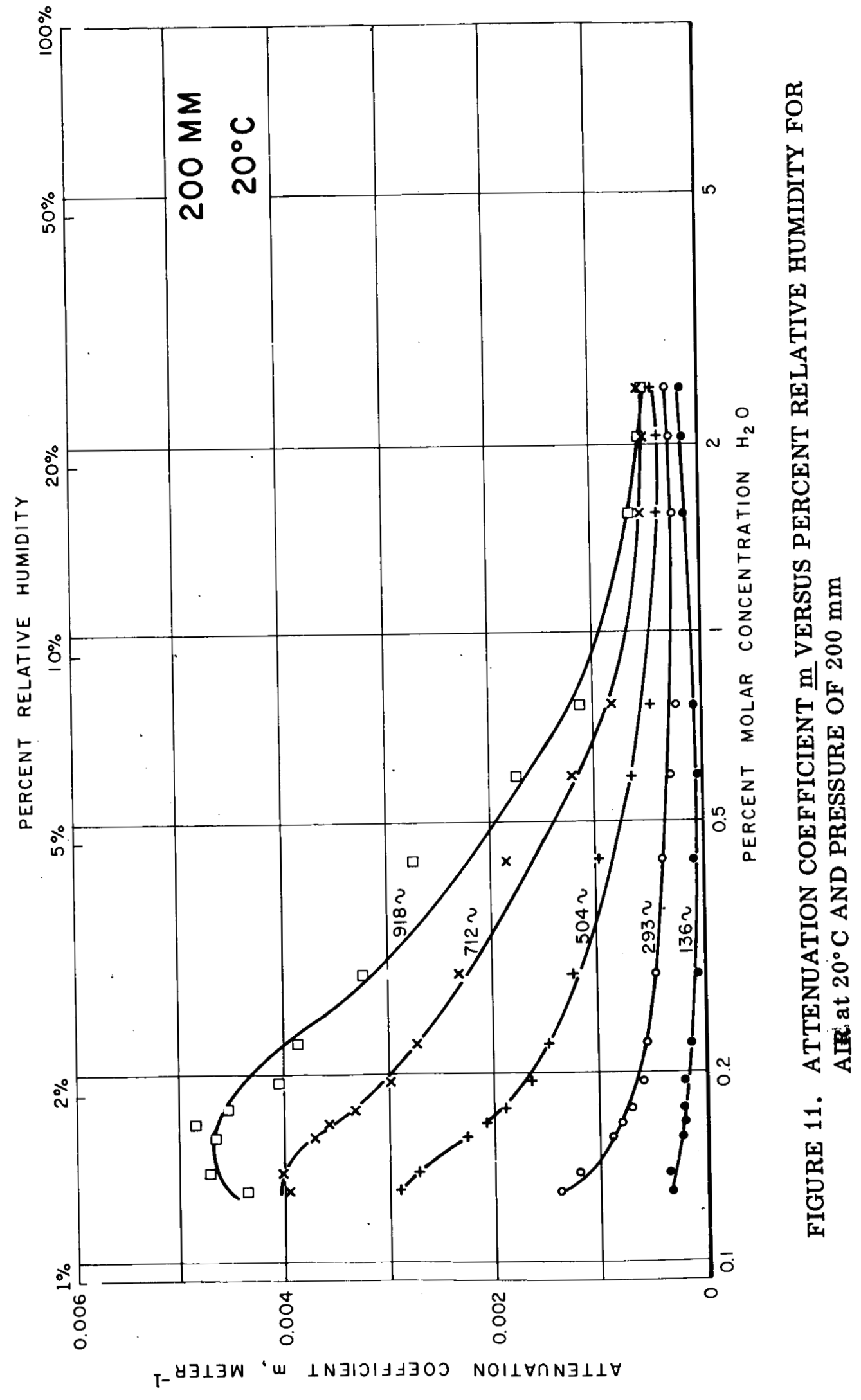




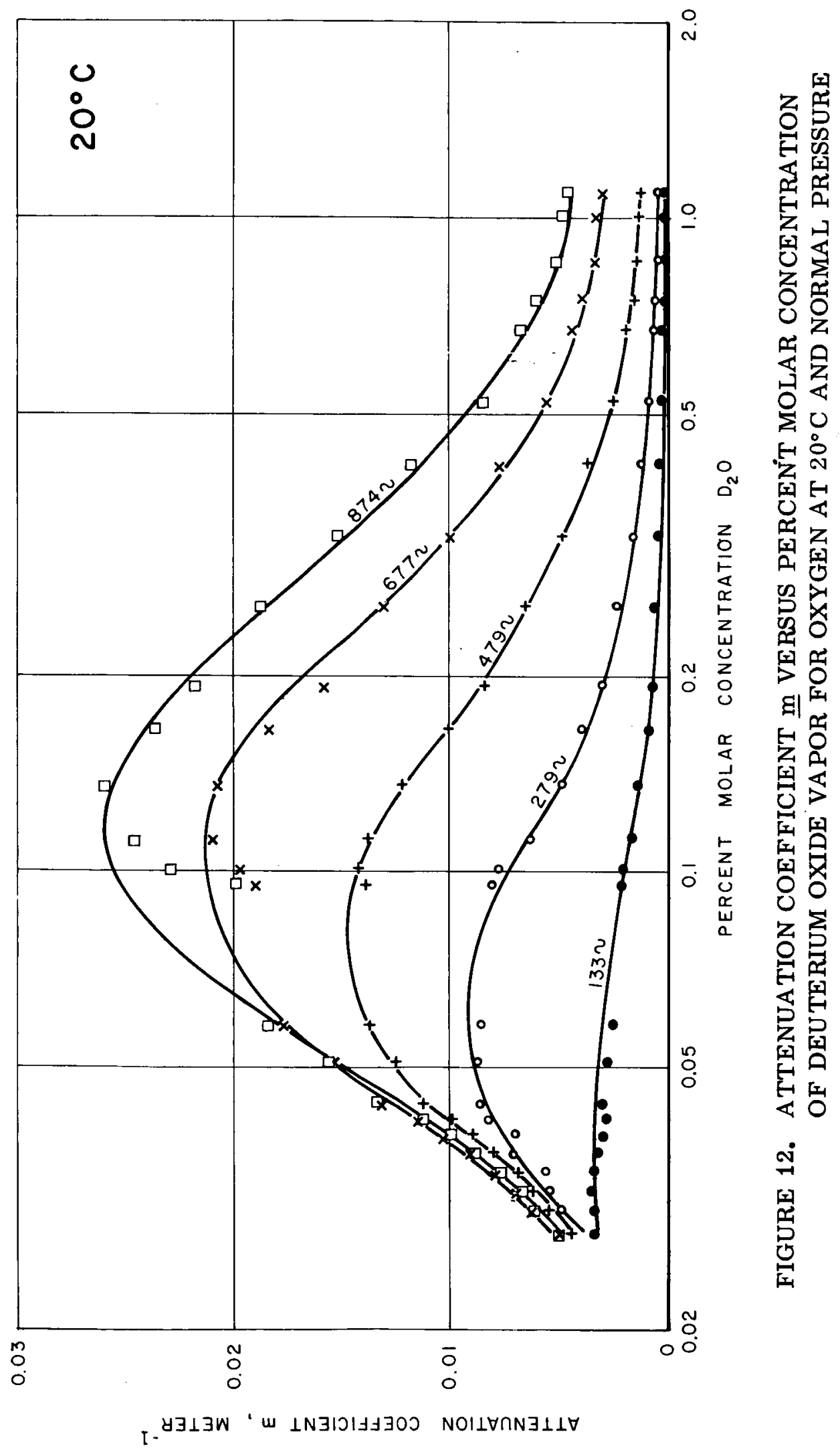




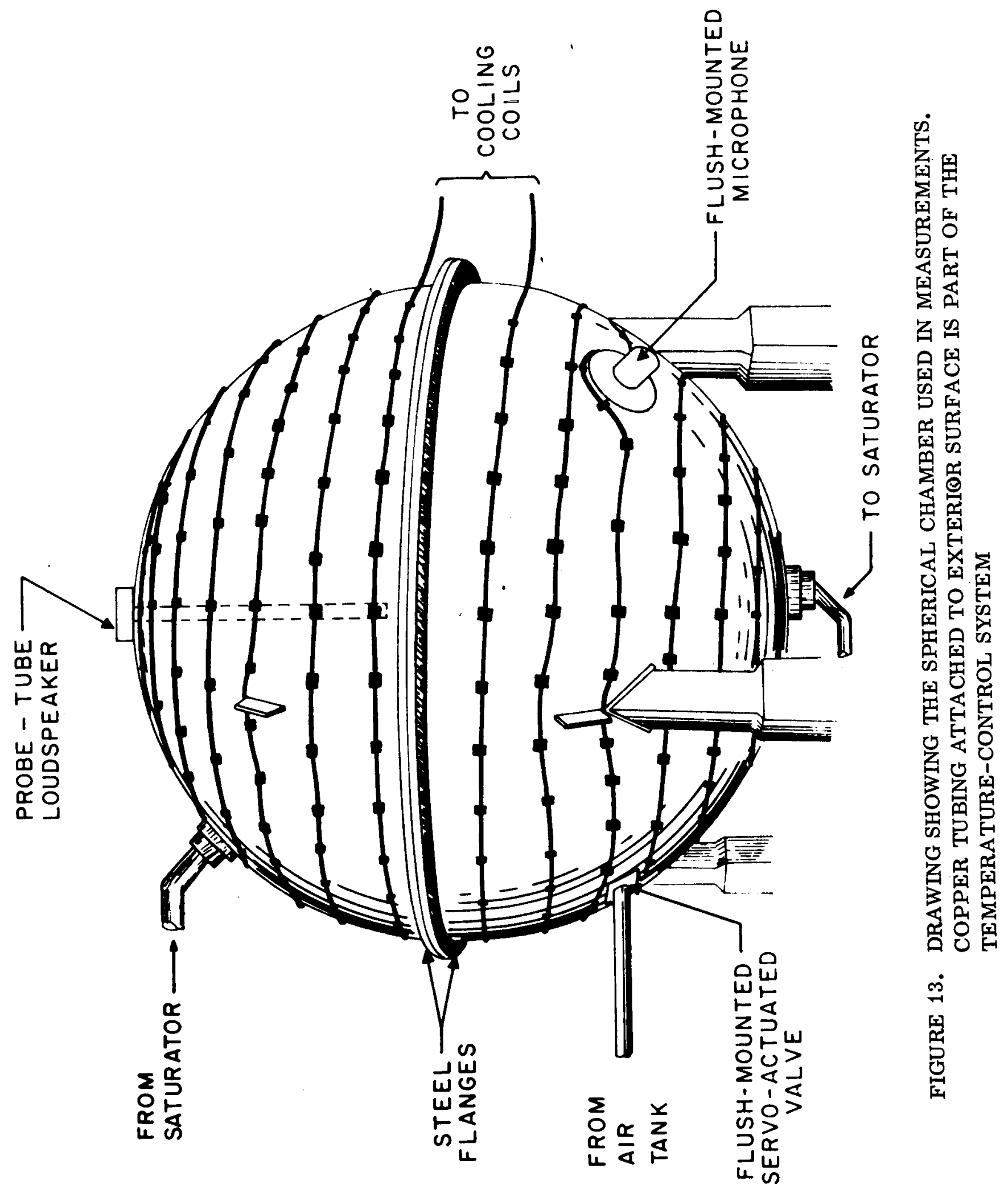




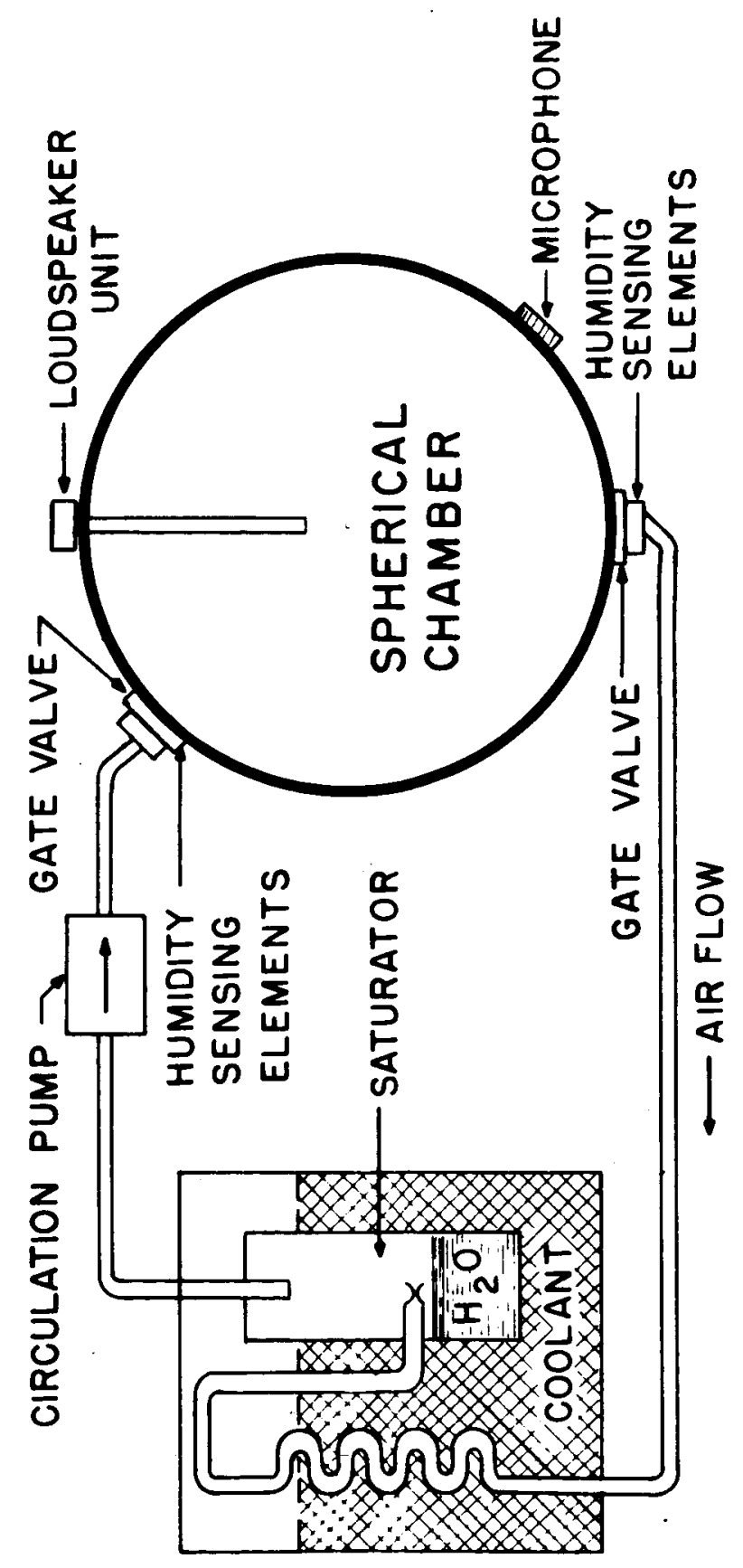

$\infty$

의 옴

是

运出斥

牙志过

勻口回

范芯安里

员层回

○国垔

田要击

为运

이일

记

鬼

넌 된

品虽

4 恐出

될 올 월

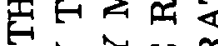

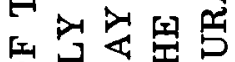

○曷是是

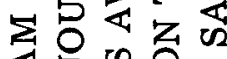

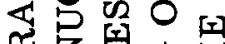

总罗

西云

完它会是

이면

H朂品

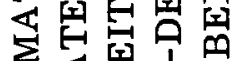

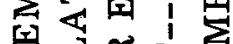

牙罗岳兵

记甚O

目它它的之

됩엉

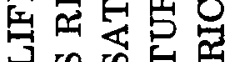

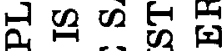

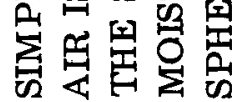

芒 


\section{REFERENCES}

1. Harris, C. M., J. Acoust. Soc. Am., 35, 11-17 (1963).

2. Kneser, H. O. , Ergeb. exact. Naturw. , 22, 121-185 (1949).

3. Delsasso, L. P., Air Force Contract W28 (099)-ac-228 (1953).

4. Knudsen, V. O., J. Acoust. Soc. Am., 5, 112-121 (1933).

5. Knudsen, V. O., and Obert, L., J. Acoust. Soc. Am., 7, 249-253 (1936).

6. Knotzel, H. and Knotzel, L., Ann. Phys. Leipz., 2, 393-403 (1948).

7. Parker, J. G., J. Chem. Phys., 34, 1763 (1961).

8. Holmes, R., Smith, F. and Tempest, W., Proc. Phys. Soc., 81, 311-319 (1963).

9. Henderson, M. C., J. Acoust. Soc. Am., 34, 349-350 (1962).

10. Clark, A. and Henderson, M. C., J. Acoust. Soc. Am., 35, 1909 (A) (1964).

11. Harlow, R. G. and Kitching, R., J. Acoust. Soc. Am., 36, 1100 (1964). 